Florida International University

FIU Digital Commons

$11-5-2018$

\title{
Folding Analysis of Reduced Bovine Pancreatic Trypsin Inhibitor (BPTI) with Aromatic Thiols and Disulfides In Vitro
}

\author{
Na Zhang \\ Florida International University, nzhan002@fiu.edu
}

Follow this and additional works at: https://digitalcommons.fiu.edu/etd

Part of the Biochemistry, Biophysics, and Structural Biology Commons, and the Biotechnology Commons

\section{Recommended Citation}

Zhang, Na, "Folding Analysis of Reduced Bovine Pancreatic Trypsin Inhibitor (BPTI) with Aromatic Thiols and Disulfides In Vitro" (2018). FIU Electronic Theses and Dissertations. 3903.

https://digitalcommons.fiu.edu/etd/3903

This work is brought to you for free and open access by the University Graduate School at FIU Digital Commons. It has been accepted for inclusion in FIU Electronic Theses and Dissertations by an authorized administrator of FIU Digital Commons. For more information, please contact dcc@fiu.edu. 


\section{FLORIDA INTERNATIONAL UNIVERSITY}

Miami, Florida

FOLDING ANALYSIS OF REDUCED BOVINE PANCREATIC TRYPSIN

INHIBITOR (BPTI) WITH AROMATIC THIOLS AND DISULFIDES IN VITRO

A dissertation submitted in partial fulfillment of

the requirements for the degree of

DOCTOR OF PHILOSOPHY

in

BIOCHEMISTRY

by

Na Zhang

2018 
To: Dean Michael R. Heithaus

College of Arts, Sciences and Education

This dissertation, written by Na Zhang, and entitled Folding Analysis of Reduced Bovine Pancreatic Trypsin Inhibitor (BPTI) with Aromatic Thiols and Disulfides In Vitro, having been approved in respect to style and intellectual content, is referred to you for judgment.

We have read this dissertation and recommend that it be approved.

Bruce R. McCord

Xiaotang Wang

Bernard S. Gerstman

Watson J. Lees, Major Professor

Date of Defense: November 5, 2018

The dissertation of Na Zhang is approved.

Dean Michael R. Heithaus College of Arts, Sciences and Education

Andrés G. Gil Vice President for Research and Economic Development and Dean of the University Graduate School

Florida International University, 2018 
C Copyright 2018 by Na Zhang

All rights reserved. 


\section{DEDICATION}

This dissertation is dedicated to my father Jigang, mother Lijun, brother Kai, and all my family members. Special thanks to my uncle Jiyuan and aunt Hong. Without their love, support, understanding and encouragement, it would not have been possible for me to complete this work.

光阴荏苒, 岁月如梭, 孤身求学于万里之外, 已五年有余。幸而父母支持, 亲 友鼓励, 终得以完成学业。此情深意长, 感激涕零! 


\section{ACKNOWLEGMENTS}

I am very grateful that so many people gave me help and encouragement in my Ph.D. study at FIU. I would like to thank all of you from the bottom of my heart.

First of all, I would like to thank my major professor, Dr. Watson J. Lees for providing me the opportunity to be a part of his research group and for his continuous help, support and guidance. He helped me in all the time of my research and writing of this dissertation. I will always be grateful for his mentorship. Thank my co-major professor, Dr. Bruce R. McCord for his great help with all the work on capillary electrophoresis. He is always kind and willing to give me insightful thoughts. I would like to extend my thanks to my

dissertation committee members, Dr. Xiaotang Wang and Dr. Bernard S. Gerstman, for their guidance and great advice for my research project.

I also want to thank all of my group members: Dr. Ram Marahatta for the synthesis work of the project, Dr. Yingsong Wang, Dr. Changjun Fan, Samina Yasmeen and Michelle Moats, for their great help and support. I gratefully thank all faculty and staff in the Department of Chemistry and Biochemistry at FIU. Special thanks to Dr. Xiaotang Wang and Dr. Yuan Liu for their great advice in both my study and personal life. Thank all my friends at FIU for their help and company.

Great thanks to the Graduate School and Department of Chemistry and Biochemistry for the teaching assistantship support, and the College of Arts, Sciences and education, Graduate and Professional Student Committee (GPSC) and SoFLACS Graduate Travel Award for the conference travel funding. 


\begin{abstract}
OF THE DISSERTATION
FOLDING ANALYSIS OF REDUCED BOVINE PANCREATIC TRYPSIN

INHIBITOR (BPTI) WITH AROMATIC THIOLS AND DISULFIDES IN VITRO

by
\end{abstract}

Na Zhang

Florida International University, 2018

Miami, Florida

\title{
Professor Watson J. Lees, Major Professor
}

Almost all therapeutic proteins contain disulfide bonds to stabilize their native structure. Recombinant DNA technology enables many therapeutic proteins to be produced in bacteria, but the expression of native proteins is not always efficient due to the limited ability of bacteria to form disulfide bonds in vivo. It is often necessary to employ in vitro oxidative folding process to form the native disulfide bonds to obtain the native structure of disulfide-containing proteins. Aromatic disulfides are small molecules designed to match some of the physical properties of the active site of protein disulfide isomerase (PDI), which catalyzes the folding process of disulfide-containing proteins in eukaryotes.

Three aromatic thiols with varying charges, PA, SA and QAS thiol, were used to fold reduced BPTI in vitro. Bovine pancreatic trypsin inhibitor (BPTI) is positively charged (pI $=10.5)$ at $\mathrm{pH} 7.3$, and we hypothesized that mixed disulfide intermediates formed between BPTI and negatively charged small molecule thiols were more likely to precipitate due to their minimized net charge. Protein precipitation was observed during folding with negatively charged thiols, PA and SA, but not positively charged thiol QAS. At the folding $\mathrm{pH}$ of 7.3 , almost $90 \%$ of native BPTI was produced in $2 \mathrm{~h}$ with the conditions of $0.25 \mathrm{mM}$ 
QAS disulfide and $10 \mathrm{mM}$ QAS thiol. Only 25\% of native BPTI was produced in $2 \mathrm{~h}$ with the best conditions for glutathione and glutathione disulfide. Aromatic thiols with an elongated alkyl group on the aromatic ring, butyl, hexyl and octyl thiol, were hypothesized to increased interactions with the hydrophobic core of disulfide-containing proteins during folding, allowing more facile access to buried disulfide bonds. However, the longer the hydrocarbon chain, the more likely protein precipitation was to occur. About $90 \%$ native BPTI was formed in $1 \mathrm{~h}$ with $0.25 \mathrm{mM}$ hexyl disulfide and $10 \mathrm{mM}$ hexyl thiol. A method using capillary electrophoresis (CE) to analysis the oxidative folding process of reduced BPTI with small molecule thiols and disulfides was also developed. Folding of reduced BPTI with QAS disulfide was analyzed using CE in a shorter run time. The consumption of protein samples and solvent solutions was minimized. 


\section{TABLE OF CONTENTS}

1 Background

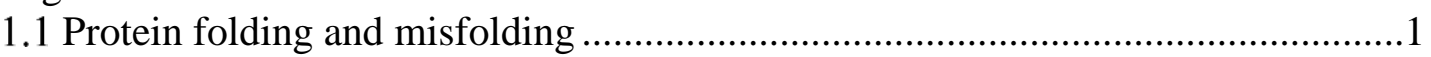

1.1.1 The mechanism of protein folding .........................................................

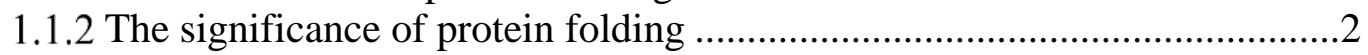

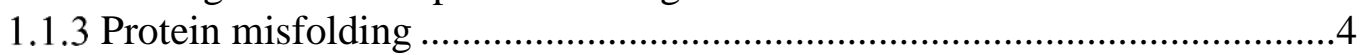

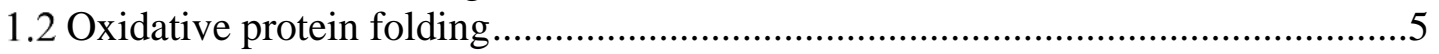

1.2.1 Disulfide bond formation ......................................................................

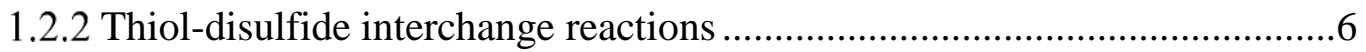

1.3 Oxidative protein folding in vivo .....................................................................

1.3.1 Oxidative protein folding in prokaryotic cells...........................................

1.3.2 Oxidative protein folding in eukaryotic cells ..............................................

1.4 Oxidative protein folding in vitro ………………..........................................10

1.4.1 Traditional small molecule thiols and disulfides......................................10

1.4.2 Selenoglutathione and the derivatives .....................................................12

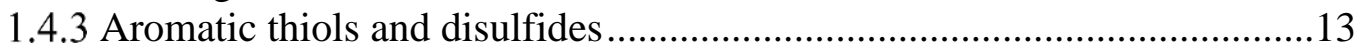

1.5 In vitro oxidative folding of disulfide-containing proteins.....................................15

1.5.1 Folding of ribonuclease A (RNase A) ..................................................15

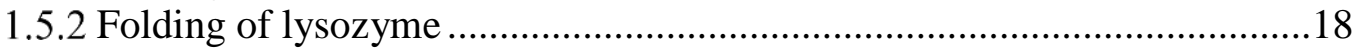

1.6 In vitro oxidative folding of bovine pancreatic trypsin inhibitor (BPTI) .............21

1.6.1 Folding of BPTI with traditional small molecule thiols and disulfides......21

1.6.2 Folding of BPTI with selenoglutathione (GSeSeG) .................................26

1.7 Experimental methods used in protein folding analysis ......................................28

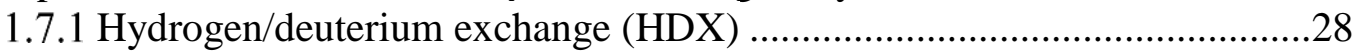

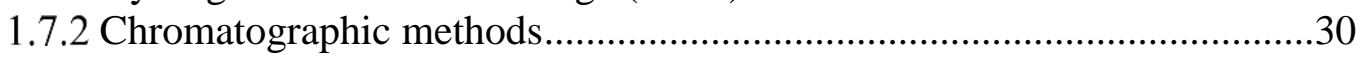

1.7.3 Capillary electrophoresis $(\mathrm{CE})$ ……………………...............................

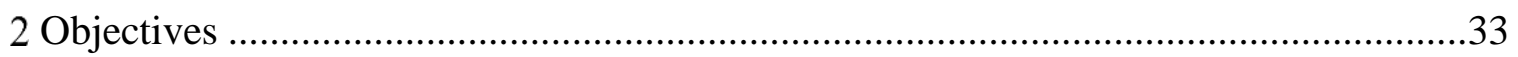

3 Investigation of charge effects of aromatic thiols on reduced BPTI folding ...................35

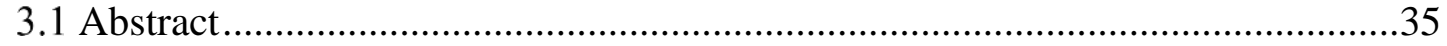

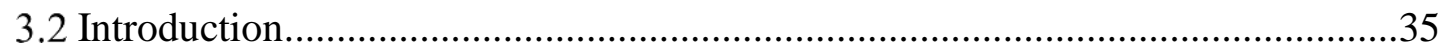

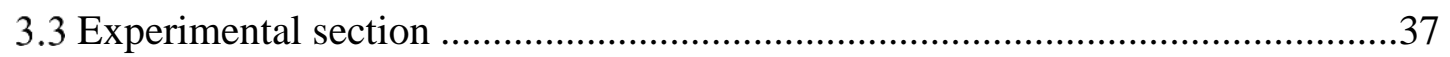

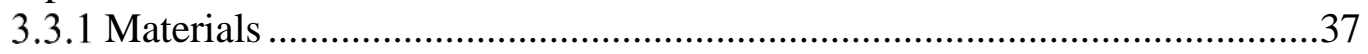

3.3.2 Preparation of reduced BPTI .................................................................. 38

3.3.3 Preparation of SA, PA and QAS thiols and disulfides ...............................39

3.3.4 Folding of reduced BPTI with aromatic thiols and disulfides......................40

3.4 Results and discussion .................................................................................. 41

3.4.1 Folding of reduced BPTI with QAS thiol and disulfide .............................41

3.4.2 Folding of reduced BPTI with PA thiol and disulfide.................................49

3.4.3 Folding of reduced BPTI with SA thiol and disulfide................................51

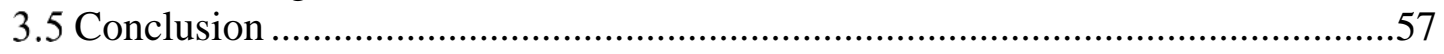


4 Investigation of hydrophobicity effects of aromatic thiols on reduced BPTI folding ....60

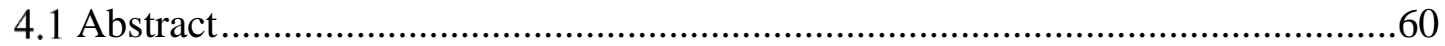

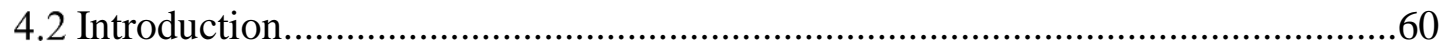

4.3 Experimental section ..............................................................................62

4.3.1 Preparation of butyl, hexyl, and octyl thiols and disulfides .......................62

4.3.2 pKa determination of three aromatic thiols using UV-vis method..............62

4.3.3 Folding of reduced BPTI with aromatic thiols and disulfides.....................63

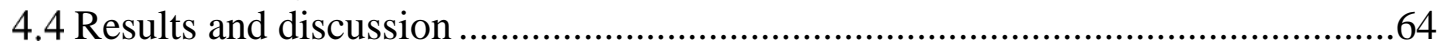

4.4.1 pKa determination of aromatic thiols .........................................................64

4.4.2 Folding of reduced BPTI with octyl thiol and disulfide..............................66

4.4.3 Folding of reduced BPTI with hexyl thiol and disulfide .............................69

4.4.4 Folding of reduced BPTI with butyl thiol and disulfide..............................74

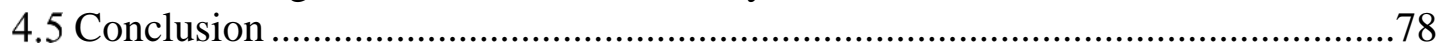

5 Oxidative folding of reduced BPTI using aromatic thiols with varying pKa values......82

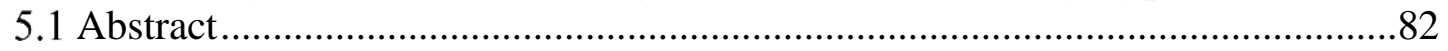

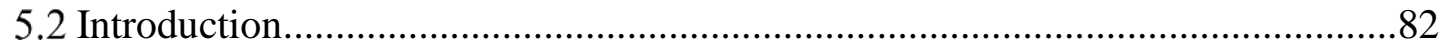

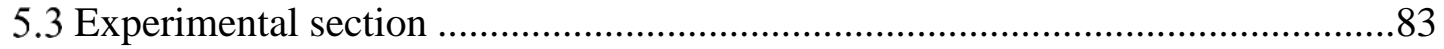

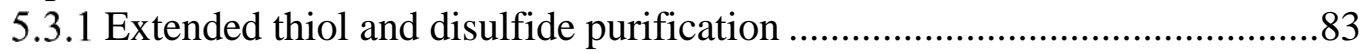

5.3.2 pKa determination of extended thiol ........................................................84

5.3.3 Folding of reduced BPTI with extended thiol and disulfide .......................85

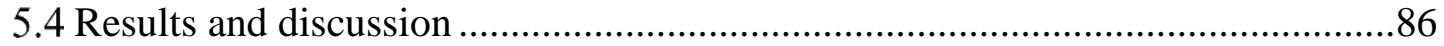

5.4.1 Extended thiol pKa determination .............................................................86

5.4.2 Folding of reduced BPTI with extended thiol and disulfide .......................87

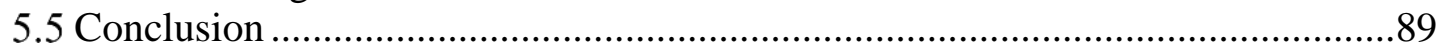

6 Folding analysis of reduced BPTI using capillary electrophoresis................................91

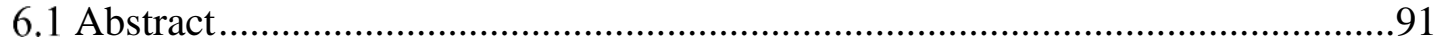

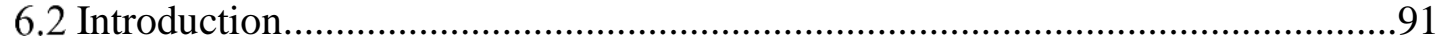

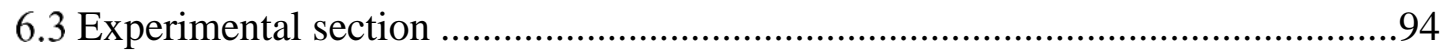

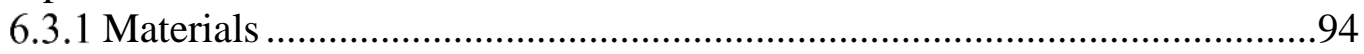

6.3.2 Protein separation using CE-UV .............................................................94

6.3.3 Protein folding analysis of reduced BPTI using CE-UV .............................95

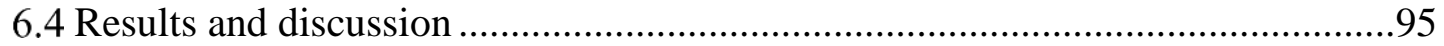

6.4.1 Protein separations using phosphate buffers ( $\mathrm{pH}$ 2.5) ...............................95

6.4.2 Protein separations using phosphate buffers ( $\mathrm{pH} 2.5$ ) with additives ........98

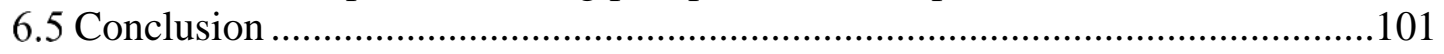

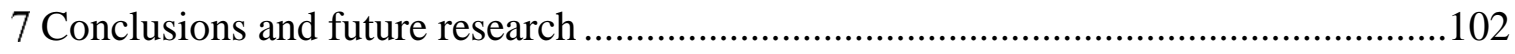

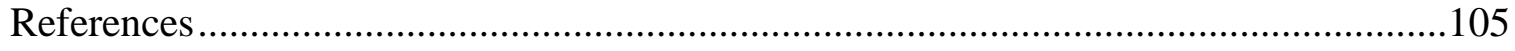

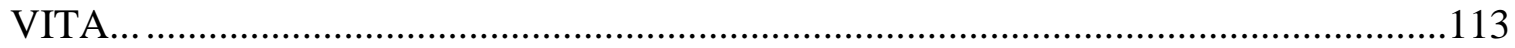




\section{LIST OF TABLES}

TABLE

PAGE

Table 1 Folding of reduced BPTI with QAS disulifide and thiol ............................... 49

Table 2 Folding of reduced BPTI with PA disulifde and thiol ..................................... 51

Table 3 Folding of reduced BPTI with SA disulifde and thiol analyzed with the 110

min method

Table 4 Folding of reduced BPTI with SA disulifde and thiol analyzed with the $40 \mathrm{~min}$ method.....

Table 5 Folding of reduced BPTI with octyl disulifde and thiol ................................ 69

Table 6 Folding of reduced BPTI with hexyl disulfide and thiol ............................... 71

Table 7 Folding of reduced BPTI with butyl disulfide and thiol ................................. 76

Table 8 Folding of reduced BPTI with extended disulifde and thiol............................ 89 


\section{LIST OF FIGURES}

FIGURE

PAGE

Figure 1 Four levels of protein organization............................................................ 1

Figure 2 Protein expression in E. coli ........................................................................ 3

Figure 3 Mechanism of thiol-disulfide interchange reaction ...................................... 6

Figure 4 Protein disulfide formation through intramolecular (a) and intermulecular (b) thiol-disulfide interchange reaction ............................................................... 7

Figure 5 PDI-catalyzed protein disulfide bond formation and isomerization................... 9

Figure 6 Structures of glutathione (GSH) and glutathione disulfide (GSSG) ................ 11

Figure 7 Structures of reduced and oxidized dithiothreitol (DTT) .............................. 12

Figure 8 Structures of selenoglutathione and selenocystamine .................................. 13

Figure 9 Thiol-disulfide interchanges of a protein with aromatic thiols and disulfides ... 14

Figure 10 Structure of bovine pancreatic ribonuclease A (RNase A) (PDB: 7RSA) ....... 16

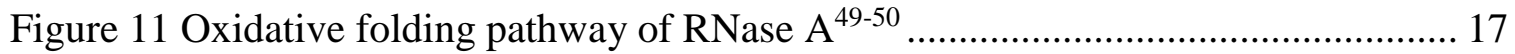

Figure 12 Structure of hen-egg-white lysozyme (PDB: 6LYZ) .................................. 19

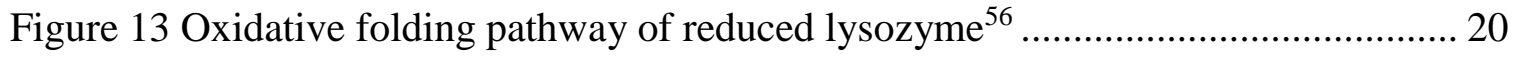

Figure 14 Structure of bovine panreatic trypsin inhibiotr (BPTI) (PDB: 1BPI) ............. 21

Figure 15 Oxidative folding pathway of reduced BPTI developed by Creighton's

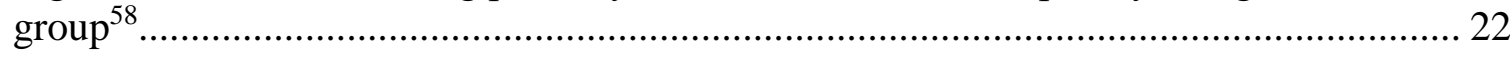

Figure 16 Oxidative folding pathway of reduced BPTI developed by Kim's group ${ }^{37} \ldots . .24$

Figure 17 Oxidative folding of N' via growth type and rearragment type pathway ${ }^{61} \ldots . . .25$

Figure 18 Aromatic thiols SA, PA and QAS and their corresponding disulfides............ 37

Figure 19 Folding of reduced BPTI with QAS disulfide only .................................. 42

Figure 20 HPLC chromatograms of reduced BPTI folding with QAS disulfide and thiol analyzed with the $110 \mathrm{~min}$ method 
Figure 21 Folding of reduced BPTI with QAS disulfide and thiol analyzed with the 110 min method

Figure 22 Folding intermediates of reduced BPTI with $0.25 \mathrm{mM}$ QAS disulfide and thiol.....

Figure 23 HPLC chromatograms of reduced BPTI folding with 5 mM GSSG and 5 $\mathrm{mM}$ GSH analyzed by the 40 min method.

Figure 24 HPLC chromatograms of reduced BPTI folding with 0.09 mM QAS

disulfide and thiol analyzed with the 40 min method..... 46

Figure 25 HPLC chromatograms of reduced BPTI folding with $0.25 \mathrm{mM}$ QAS

disulfide and thiol analyzed with the 40 min method 47

Figure 26 Folding of reduced BPTI with QAS disulfide and thiol analyzed with the 40 min method 48

Figure 27 HPLC chromatograms of reduced BPTI with PA disulfide and thiol ............. 50

Figure 28 Folding of reduced BPTI with 0.09 mM PA disulfide and thiol ...................... 50

Figure 29 Folding of reduced BPTI with 0.25 mM SA disulfide ................................. 52

Figure 30 Folding of reduced BPTI with 0.125 mM SA disulfide and thiol ................... 52

Figure 31 HPLC chromatograms of reduced BPTI with SA disulfide and thiol ............. 54

Figure 32 Folding of reduced BPTI with SA disulfide and thiol analyzed with the 110

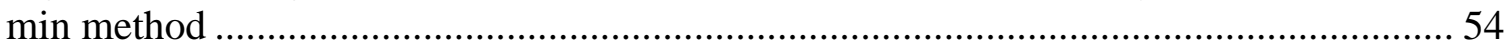

Figure 33 Folding intermediates of reduced BPTI folding with SA disulfide and thiol... 55

Figure 34 Folding of reduced BPTI with SA disulfide and thiol analyzed with the 40

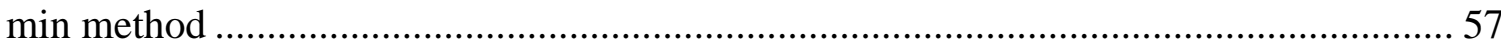

Figure 35 Comparison of reduced BPTI folding with GSSG/GSH and QAS

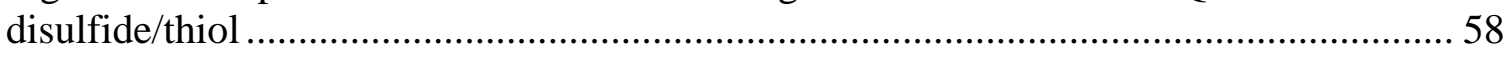

Figure 36 The charge effect of small molecules on the folding of reduced BPTI........... 59

Figure 37 Structures of aromatic butyl, hexyl, and octyl thiols and disulfides ............... 61

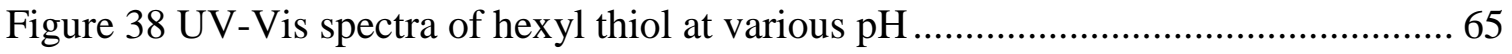

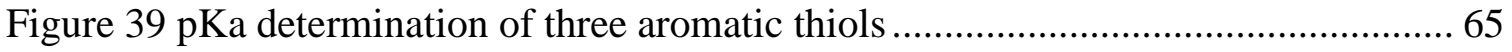

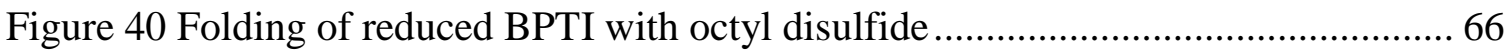


Figure 41 HPLC chromatograms of reduced BPTI folding with octyl disulfide and thiol67

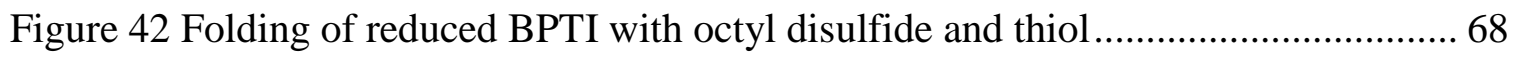

Figure 43 Folding of reduced BPTI with hexyl disulfide ........................................... 70

Figure 44 HPLC chromatograms of reduced BPTI with hexyl disulfide and thiol ......... 71

Figure 45 Folding of reduced BPTI with hexyl disulfide and thiol ............................. 72

Figure 46 Folding of reduced BPTI with $2 \mathrm{mM}$ hexyl thiol and disulfide ..................... 74

Figure 47 Initial folding rates of reduced BPTI with $2 \mathrm{mM}$ hexyl thiol and disulfide ..... 74

Figure 48 Folding of reduced BPTI with butyl disulfide and thiol............................... 75

Figure 49 HPLC chromatograms of reduced BPTI folding with butyl disulfide and

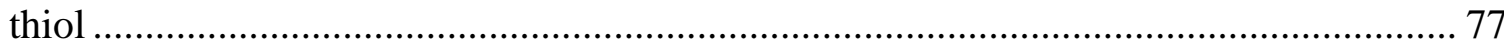

Figure 50 Folding intermediate formation of reduced BPTI with butyl disulfide and

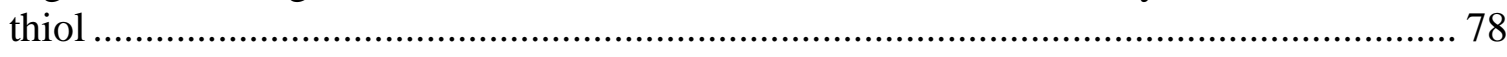

Figure 51 Reduced BPTI folding with aromatic thiols/disulfides and GSH/GSSG ........ 79

Figure 52 The effect of n-alkyl chain length of small molecule thiols and disulfides on

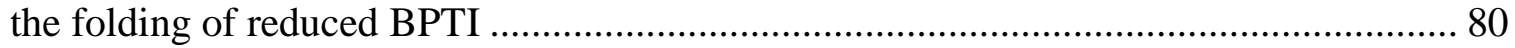

Figure 53 Folding intermdiates of reduced BPTI with aromatic thiols and disulfides,

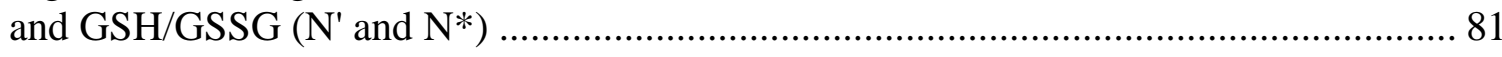

Figure 54 Structures of extended thiol and its disulfide ........................................ 83

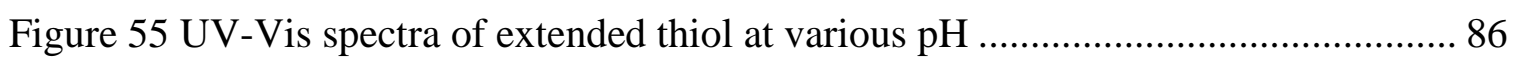

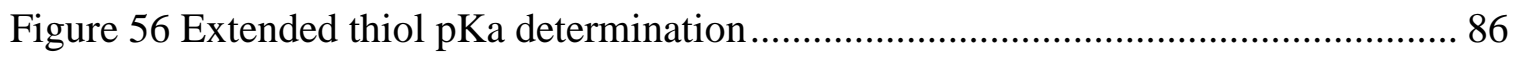

Figure 57 HPLC chromatograms of reduced BPTI folding with extended disulfide ....... 87

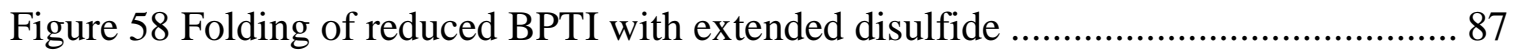

Figure 59 HPLC chromatograms of reduced BPTI folding with $0.09 \mathrm{mM}$ extended

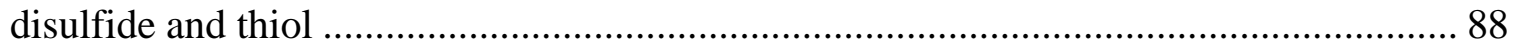

Figure 60 Folding of reduced BPTI with extended disulfide and thiol ......................... 88

Figure 61 Electropherograms of native and reduced BPTI .................................... 96

Figure 62 Electropherograms of native BPTI using phosphate buffers......................... 97 
Figure $63 \mathrm{CE}$ analysis of native BPTI using phosphate buffers

Figure 64 Electropherograms of reduced BPTI folding with $0.25 \mathrm{mM}$ QAS disulfide.... 98

Figure 65 Electropherograms of native BPTI using phosphate buffers with 0.1\% HEC. 99

Figure 66 Native BPTI analysis using phosphate buffers with HEC.

Figure 67 Electropherograms of reduced BPTI folding with $0.25 \mathrm{mM}$ QAS disulfide

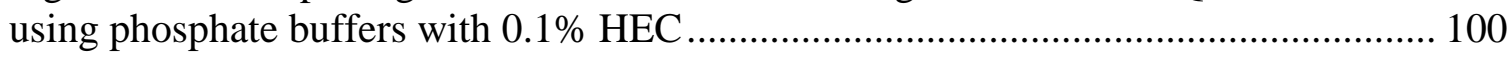

Figure 68 Folding analysis of reduced BPTI with 0.25 mM QAS disulfide ................. 100 


\section{LIST OF ABBREVIATIONS}

BPTI

CE

CIEF

CZE

DTT

Des species

EACA

EDTA

EOF

$\mathrm{GdnHCl}$

GSH

6GSSG

HDX

HEC

IAA

IAM

IEC

MS

NMR

PA thiol

PDI
Bovine pancreatic trypsin inhibitor

Capillary electrophoresis

Capillary isoelectric focusing

Capillary zone electrophoresis

Dithiothreitol

Protein folding intermediate lacking one native disulfide bond

€-Amino-N-caproic acid

Ethylenediaminetetraacetic acid

Electroosmotic flow

Guanidine hydrochloride

Glutathione

Glutathione disulfide

Hydrogen/deuterium exchange

Hydroxyethyl cellulose

Iodoacetate

Iodoacetamide

Ion exchange chromatography

Mass spectrometry

Nuclear magnetic resonance

Phosphoric acid thiol

Protein disulfide isomerase 
pI

QAS thiol

RP-HPLC

SA thiol

$\mathrm{scFv}$

SEC

TFA
Isoelectric point

Quaternary ammonium salt thiol

Reversed phase-high performance liquid chromatography

Sulfonic acid thiol

Single-chain variable fragment

Size exclusion chromatography

Trifloroacetic acid 


\section{Background}

\subsection{Protein folding and misfolding}

Proteins are large organic molecules universally found in all living organisms and are crucial to almost all biological processes, including catalyzing enzymatic reactions, transporting other molecules, and providing immune protection. ${ }^{1}$ Proteins have four levels of organization called primary, secondary, tertiary, and quaternary structures. The primary structure of a protein is simply a polypeptide chain composed of a series of amino acid residues linked by peptide bonds in a specific order. The secondary structure refers to local folding of the polypeptide chains into coils or sheets by hydrogen bonding interactions such as $\alpha$-helixes and $\beta$-sheets. The tertiary structure is the three-dimensional structure of a polypeptide chain. The distribution of amino acids shows that the interior of a cytosolic protein consists of nonpolar residues with the hydrophobic side chains buried inside the protein. If a protein consists of more than one polypeptide chain, each polypeptide chain is considered as a protein subunit and the interaction between subunits results in the formation of a specific three dimensional spatial arrangement called the quaternary structure.

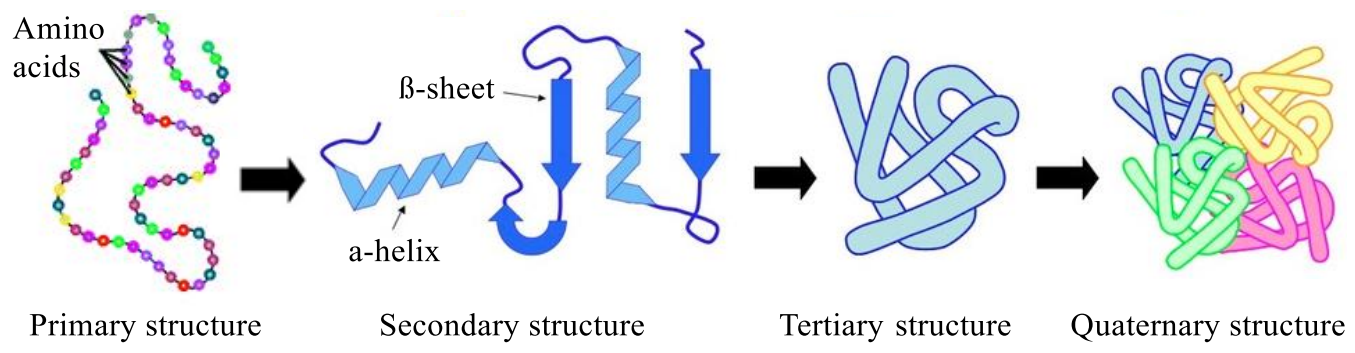

Figure 1 Four levels of protein organization

\subsubsection{The mechanism of protein folding}

Most proteins have a specific three-dimensional structure as their biologically functional structure. Protein folding is the process by which a polypeptide chain forms its 
functional three-dimensional structure from a random coil through a series of conformational changes. ${ }^{2}$ Protein folding kinetics can be described by a rugged energy landscape which has many local energy minima and maxima. ${ }^{3}$ In order to obtain the native conformation of the protein, the polypeptide chain needs to acquire enough energy to overcome any kinetic energy barrier. Theoretical and experimental results have revealed that only a limited number of all possible conformations can be formed during the folding process. Some folding intermediates get caught into local minima, which are considered as kinetic traps.

The formation of the correctly folded protein is related to its amino acid sequence as well as the folding environment, which in most cases is the cellular milieu. ${ }^{4}$ The primary structure information of a protein determines the possible folding pathways to the native conformation. Any changes in the folding environment can also change the favored folding pathway and in some cases can lead to protein misfolding. In most cases protein folding is spontaneous and involves different types of forces including non-covalent forces (e.g., hydrophobic interactions, hydrogen bonding and van der Waals forces) and covalent disulfide bonds. ${ }^{5}$ Disulfide bonds are the only covalent bonds formed during folding, and they play an important role in stabilizing the native structure of many proteins.

\subsubsection{The significance of protein folding}

Therapeutic proteins are revolutionizing the treatment of a wide range of diseases. ${ }^{6}$ In 2016, ten protein-based therapeutics were amongst the top 25 best-selling drugs. More than 200 therapeutic peptides and proteins have been approved by the FDA for clinical use including insulin and Herceptin ${ }^{\circledR}$. With the development of recombinant DNA technology, numerous therapeutic proteins can be efficiently overexpressed in Escherichia coli. ${ }^{7}$ 
Escherichia coli has been considered as a major expression host for recombinant protein production since it has relatively simple, cost-effective, and well-characterized genetics. ${ }^{8}$ During overexpression, proteins, especially disulfide-containing ones, tend to misfold and aggregate, forming inclusion bodies which mainly contain inactive proteins. The formation of inclusion bodies has some advantages, as it allows bacteria to produce high expression levels of the targeted protein efficiently, and helps to protect the expressed protein from being degraded by cellular proteases. However, in many cases the lack of certain posttranslational modification abilities, and insufficiencies in disulfide bond formation, prohibits E. coli from producing the biologically functional proteins. ${ }^{9}$ In order to obtain the active form of the protein, inclusion bodies need to be resolubilized with denaturants and the resulting proteins refolded to the native form under appropriate in vitro conditions (Figure 2). ${ }^{10}$ Various methods have been adopted to produce the folded structures of proteins in vitro from inclusion bodies with a reasonable yield.

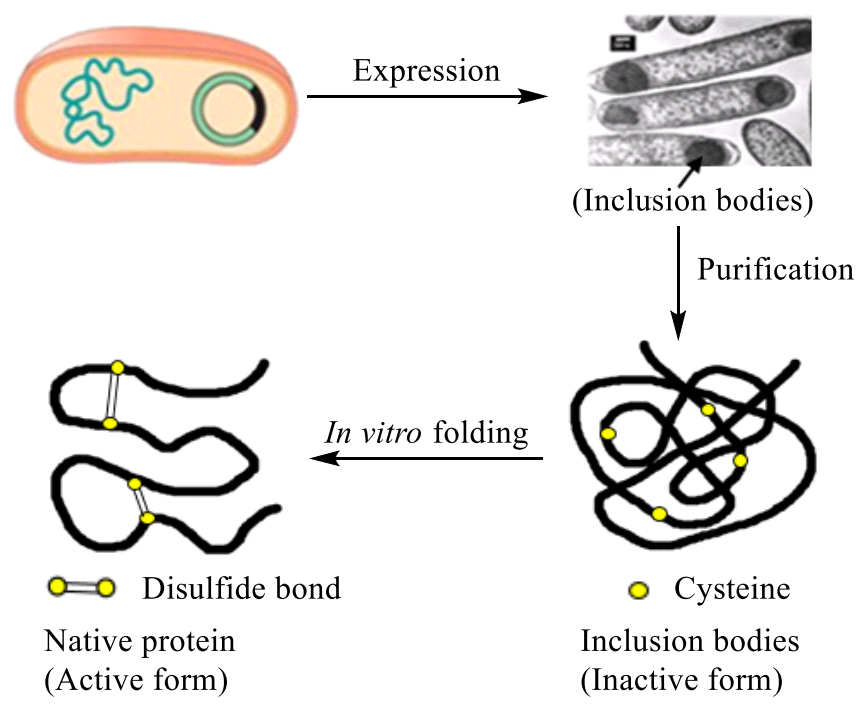

Figure 2 Protein expression in E. coli 
Almost all pharmaceutically relevant proteins contain disulfide bonds which help to stabilize the active structure of the protein. During in vitro oxidative folding of disulfidecontaining proteins, the correct disulfide bonds are formed and the protein becomes active. However, the in vitro folding process is usually time-consuming and low in yield because of slow thiol-disulfide interchange reactions. Therefore, it is important to develop an effective in vitro oxidative folding process in order to form the active protein structure.

\subsubsection{Protein misfolding}

Correctly folded proteins are essential to the regulation of almost all biological processes in living organisms. ${ }^{11}$ The biological function of a protein depends on its native three-dimensional structure. Environmental factors or genetic mutations may alter a protein's three-dimensional structure, leading to protein misfolding. Misfolded proteins

may aggregate, resulting the formation of amyloid-like deposits in the cell. ${ }^{12}$ Proteins with aberrant conformations can be toxic to cells, especially neurons, and lose the biological functions of natively folded proteins. Numerous diseases have been shown to be related to protein misfolding. These diseases are grouped together as protein conformational disorders (PCDs), and examples include Alzheimer's disease (AD), Parkinson disease (PD), type II diabetes, and amyotrophic lateral sclerosis (ALS). ${ }^{13}$

More than $50 \%$ of pathologic amyloid related proteins contain disulfide bonds, providing the evidence that disulfide bond formation has an important effect on protein aggregate formation and the cytotoxicity of aggregated proteins. ${ }^{14}$ Studies of amyloid fibril formation by human lysozyme have confirmed that native disulfide bond influenced the cytotoxicity and morphology of the fibrils significantly. Disulfide bonds inhibited the aggregation of lysozyme and the resulting formation of amyloid fibrils, by stabilizing the 
folded state of the protein thermodynamically. About $25 \%$ of proteins that misfold in neurodegenerative diseases contain disulfide bonds, and most are related to systemic pathologies. It has been demonstrated that disulfide bonds coevolved with proteins in order to minimize the propensity to misfold the protein and form toxic aggregates. ${ }^{15}$ Understanding the fundamental aspect of how disulfide-containing proteins fold to the active form may help minimize the accumulation of misfolded proteins.

\subsection{Oxidative protein folding}

\subsubsection{Disulfide bond formation}

Oxidative protein folding analysis is focused on both the formation of native disulfide bonds and the conformational folding of the protein to its native three-dimensional structure. ${ }^{16}$ Disulfide folding intermediates during oxidative folding can be isolated and characterized to elucidate the oxidative folding pathways, and the process explains how disulfide-containing proteins obtain their native structure. Disulfide bonds are formed by the oxidation between two thiol groups of cysteine residues. Disulfide bonds can stabilize the native protein structure by significantly lowering the entropy of the unfolded state. ${ }^{17}$ Since the 1960s, oxidative folding studies have been conducted using many disulfidecontaining proteins on the basis of the disappearance and reformation of disulfide bonds in the protein structure. It has been shown that bovine pancreatic ribonuclease A (RNase A) can unfold completely if the four native disulfide bonds in the structure are broken, but the active form of the protein is obtained when the disulfide bonds are formed under optimal conditions. $^{18}$ 


\subsubsection{Thiol-disulfide interchange reactions}

Disulfide bond formation is a reversible process involved in stabilizing proteins, protecting proteins from oxidative damage, and regulating the biological activities of proteins. Disulfide bonds are formed via thiol-disulfide interchange reactions (Figure 3). ${ }^{16,19}$ These reactions involve the exchange of two redox equivalents, in which the thiolate anion serves as an electron donor and the disulfide as an electron acceptor. Reversible thioldisulfide interchange reactions are initiated by the nucleophilic attack of a thiolate anion $\left(\mathrm{R}_{1} \mathrm{~S}^{-}\right)$on the sulfur atom of a disulfide $\left(\mathrm{R}_{2}-\mathrm{S}-\mathrm{S}-\mathrm{R}_{3}\right)$ bond, followed by the displacement of the other sulfur atom $\left(\mathrm{R}_{3} \mathrm{~S}^{-}\right)$and the formation of a new disulfide $\left(\mathrm{R}_{1}-\mathrm{S}-\mathrm{S}-\mathrm{R}_{2}\right)$. The formation of a trisulfide anionic transition state intermediate $\left({ }^{\delta-} \mathrm{S}-\mathrm{S}-\mathrm{S}^{\delta-}\right)$ is the ratedetermining step in the thiol-disulfide exchange reaction. ${ }^{20}$ The rate of a thiol-disulfide interchange reaction is highly affected by the accessibility of the reactive groups and the distance between the two sulfur atoms for exchange to meet the steric requirement of the reaction. $^{21-22}$

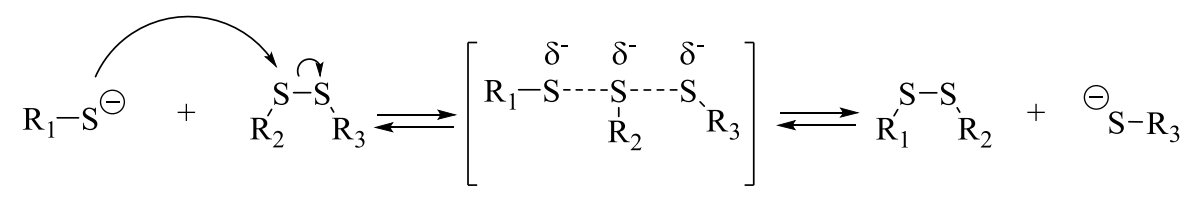

Thiolate ion Disulfide

New disulfide Leaving group

Figure 3 Mechanism of thiol-disulfide interchange reaction

The formation of protein disulfide bonds in a polypeptide can be promoted by molecular chaperones or redox reagents consisting of small molecule thiols (RSH) and disulfides (RSSR). The two classes of thiol-disulfide interchange reactions that take place during protein folding are intramolecular and intermolecular reactions (Figure 4). ${ }^{16,23}$ Intramolecular disulfide bond formation occurs between two cysteine groups within the 
polypeptide chain, in which a protein thiolate attacks a disulfide bond of the same protein, which is called a disulfide shuffling reaction. The oxidative state of the protein and the number of disulfide bonds within the protein will not change in the disulfide shuffling reaction since both participants are protein-bounded. Disulfide bonds can also be formed intermolecularly by thiol-disulfide exchanges with a redox reagent, which involves the formations of a mixed disulfide intermediate between the protein and the redox reagent. To form the protein disulfide bond, the mixed disulfide intermediate can then be attacked by another protein thiolate group to initiate an intramolecular thiol-disulfide interchange reaction. A new protein disulfide bond is formed in the redox reaction, and the oxidative state of the protein is changed.

a.
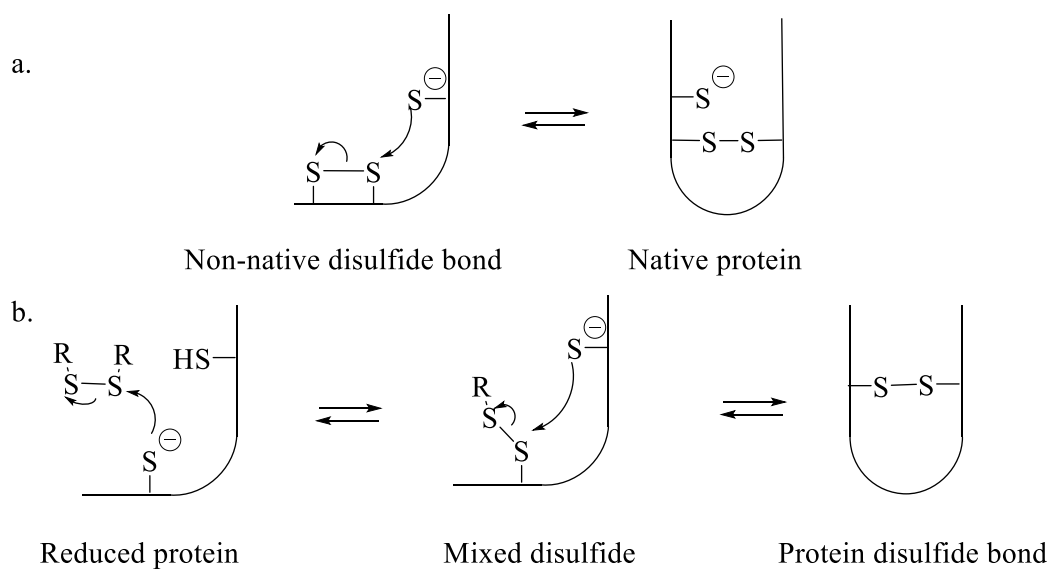

Figure 4 Protein disulfide formation through intramolecular (a) and intermulecular (b) thiol-disulfide interchange reaction

\subsection{Oxidative protein folding in vivo}

Disulfide bonds are crucial to the stability and biological functions of many extracellular proteins. In vivo oxidative folding of disulfide-containing proteins is assisted by various molecular chaperones which aid in forming native disulfide bonds in the threedimensional structure. ${ }^{24-26}$ 


\subsubsection{Oxidative protein folding in prokaryotic cells}

In bacteria, the folding of disulfide-containing proteins is facilitated by an oxidativereduction cycling process involving a series of thiol-disulfide oxidoreductases. ${ }^{27}$ Thiol oxidase disulfide bond $\mathrm{A}(\mathrm{DsbA})$ is an enzyme found in the periplasm that is responsible for disulfide bond formation. Disulfide bond A (DsbA) contains a thioredoxin-like domain with a CXXC motif in its catalytic site. ${ }^{28}$ Disulfide bond A (DsbA) has a high reactive catalytic ability that can oxidize the protein to form disulfide bonds randomly without distinguishing among cysteines. Reduced DsbA is then oxidized back to its disulfide form by DsbB through a thiol-disulfide interchange reaction. Since the formation of protein disulfide bonds catalyzed by DsbA is a rapid and indiscriminate process, non-native disulfide bonds can be formed in proteins containing multiple cysteine residues. Reduced catalytically active proteins $\mathrm{DsbC}$ and $\mathrm{DsbG}$ are found to be involved in catalyzing isomerization of non-native disulfide bonds to native disulfide bonds in the protein being folded (disulfide shuffling). ${ }^{27}$ In the isomerization pathway, DsbC needs to be reduced by membrane protein DsbD in order to maintain its active form in the periplasm.

\subsubsection{Oxidative protein folding in eukaryotic cells}

In eukaryotic cells, nascent polypeptides with cysteine residues are translocated from the cytosol to the endoplasmic reticulum (ER) which provides a more oxidizing environment to form protein disulfide bonds. The formation of disulfide bonds in the ER is catalyzed by members of the protein disulfide isomerase (PDI) family. ${ }^{29}$ PDI proteins are dithiol-disulfide oxidoreductases containing four thioredoxin (a-b-b'-a') domains forming a twisted U-shaped structure. However only the a and a' domains have the CXXC active site motifs that can facilitate disulfide bond formation and isomerization in proteins 
through thiol-disulfide interchange reactions (Figure 5). ${ }^{30}$ The N-terminal active site cysteine in the CXXC motif of PDI has a low pKa value (pKa $=6.7)$, so that the thiol group can remain in the thiolate form and have high nucleophilic activity at physiological $\mathrm{pH}^{31}$

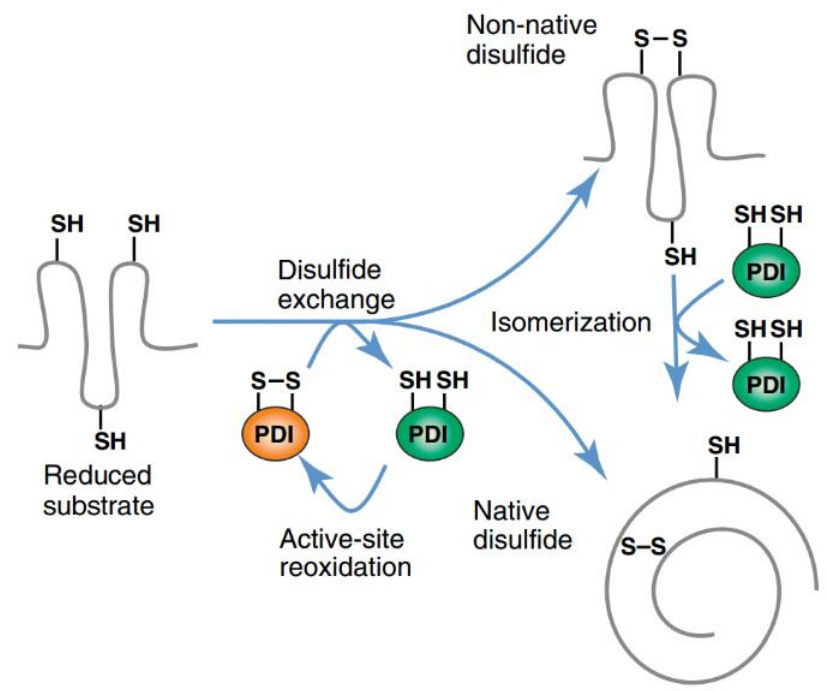

Figure 5 PDI-catalyzed protein disulfide bond formation and isomerization

Protein disulfide isomerase (PDI)-catalyzed disulfide formation occurs between oxidized PDI and the protein substrate with free thiols. Oxidized PDI with the active site cysteine in the disulfide form can directly interact with the thiol in the substrate to form a mixed disulfide intermediate between protein and PDI. The free thiol in the protein attacks the mixed disulfide to form a protein disulfide bond and reduced PDI is released. Reduced PDI can then be oxidized by cellular oxidizing agents such as ER oxidoreductin 1 protein (Ero1p) in the ER to reform oxidized PDI. ${ }^{32-33}$ During the rearrangement of non-native disulfide bonds in the protein substrate, the thiolate ion in the active site of reduced PDI acts as a nucleophile to attack the sulfur atom in the protein disulfide yielding a mixed disulfide intermediate. Then the native protein disulfide bond is produced via further thioldisulfide interchanges. 
In addition, glutathione (GSH) and glutathione disulfide (GSSG) are important redox agents in eukaryotic cells. ${ }^{34-35}$ The ratio of GSH/GSSG is about 3:1 in the ER which is more oxidizing compared to the ratio of 100:1 in the cytosol. Glutathione disulfide (GSSG) was found not to directly interact with Erop1 as a source of oxidizing equivalents in the PDI-catalyzed protein disulfide bond formation process. ${ }^{36}$ Glutathione (GSH) can reduce the disulfides in PDI and protein substrate to produce GSSG, so the redox buffer of GSH/GSSG is generated from a balance between Erolp-mediated oxidation and GSHmediated reduction in the ER, which is able to reduce and rearrange incorrect protein disulfide bonds.

\subsection{Oxidative protein folding in vitro}

Oxidative protein folding involves thiol-disulfide interchange reactions, which are usually considered as the rate-limiting steps of the process. In vitro folding of disulfidecontaining proteins is conducted in the presence of redox agents that mimic the in vivo folding environment which has a series of protein chaperones and catalysts to facilitate thiol-disulfide exchanges efficiently. Many efforts have been taken to develop redox agents to improve the folding yield and rate of disulfide-containing proteins in vitro.

\subsubsection{Traditional small molecule thiols and disulfides}

Traditionally, redox buffers containing aliphatic small molecules, such as glutathione disulfide (GSSG) and glutathione (GSH), have been widely employed to facilitate the in vitro folding of many disulfide-containing proteins (Figure 6) ${ }^{37}$ In the redox buffer, GSSG acts as a stoichiometric thiol oxidant that can interact with the free thiols of proteins to form disulfide bonds in the protein, and GSH catalyzes the rearrangement of non-native disulfide bond to native protein disulfide bonds. The optimal concentrations of GSSG and 
GSH to fold several disulfide-containing proteins efficiently has been determined in previous studies and found to be similar to the concentrations found in vivo in the ER. The folding pathways of some model proteins, such as lysozyme and RNase A, have been elucidated and demonstrated as the accumulation and rearrangement of kinetically stable folding intermediates containing various numbers of disulfide bonds using GSH and GSSG as the redox folding buffer. ${ }^{38}$

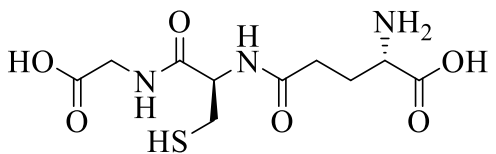

Glutathione (GSH)

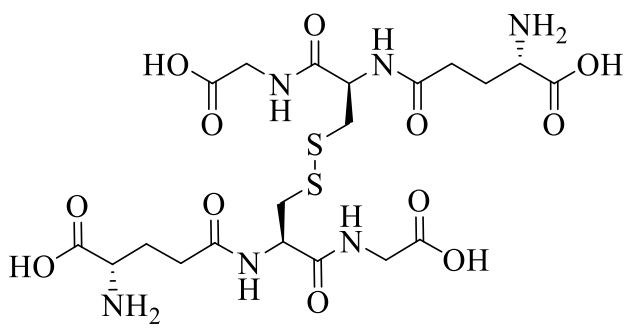

Glutathione disulfide (GSSG)

Figure 6 Structures of glutathione (GSH) and glutathione disulfide (GSSG)

Subsequent studies have used redox buffers containing reduced/oxidized dithiothreitol $\left(\right.$ DTT $^{\text {red }} / \mathrm{DTT}^{\text {ox }}$ ) to improve the oxidative folding process (Figure 7). ${ }^{22}$ Reduced dithiothreitol (DTT $\left.{ }^{\mathrm{red}}\right)$ is a strong reducing agent that can promote protein disulfide bond reduction. The formation of a disulfide bond is conducted through two steps of thioldisulfide interchange reactions. Oxidized dithiothreitol $\left(\mathrm{DTT}^{\mathrm{ox}}\right)$ can be attacked by the protein thiolate ion to form a mixed disulfide bond between protein and DTT. The mixed disulfide bond can then be attacked by another thiolate ion in the protein, leading to the formation of a protein disulfide bond. Since the thiol of DTT is more reactive than the thiol in the protein structure, DTT is more likely to close the ring rapidly leading to the formation of oxidized DTT with a disulfide-bonded closed-ring structure. Compared to GSH, the 
advantage of DTT is that oxidative folding reactions with DTT rarely accumulate mixed disulfide intermediate species. ${ }^{22,39}$

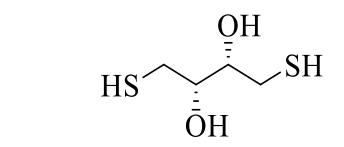

Reduced dithiothreitol (DTT ${ }^{\text {red }}$ )

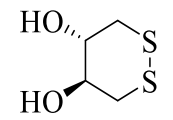

Oxidized dithiothreitol (DTT $\left.{ }^{\mathrm{ox}}\right)$

Figure 7 Structures of reduced and oxidized dithiothreitol (DTT)

\subsubsection{Selenoglutathione and the derivatives}

Besides small molecule thiols and disulfides, selenium-containing analogs such as selenoglutathione (GSeSeG) and its derivative molecules have been employed as redox buffer for in vitro oxidative protein folding (Figure 8). ${ }^{40-41}$ Selenoglutathione (GSeSeG), a selenium-containing analog of GSSG, has been successfully synthesized and characterized in previous studies. ${ }^{42}$ Diselenides are thermodynamically more stable with lower redox potentials $\left(\mathrm{E}^{\circ \prime}=-407 \mathrm{mV}\right)$ than disulfides. However, selenium has much higher polarizability compared to sulfur, making it a great electrophile in nucleophilic attack reactions for thiol-disulfide interchanges. Diselenides can be used to facilitate the isomerization of non-native disulfide bonds and kinetically trapped folding intermediates. Selenols have a low $\mathrm{pKa}(\mathrm{pKa}=5.2)$, and are completely deprotonated to the active ionized form at physiological $\mathrm{pH}^{43}$ Therefore, the use of selenium-containing analogs in redox buffers can expand the $\mathrm{pH}$ range of the folding reactions to acidic conditions.

Redox buffers containing GSeSeG and its derivatives showed enhanced folding rates and yields for many disulfide-containing proteins. ${ }^{44}$ Even a low concentration $((20 \mu \mathrm{M})$ of GSeSeG was able to fold reduced RNase A 2 times faster than the same amount of GSSG. ${ }^{42}$ Additionally, many commercially available small molecule diselenide compounds such as 
selenocystamine were also used to fold many disulfide-containing proteins both in vivo and in vitro. Redox buffers consisting of diselenides can significantly increase the oxidative folding rate and yield because of the enhanced reactivity of diselenides in thiol-disulfide interchange reactions. Diselenides can rearrange kinetically trapped intermediates to native protein faster compared to their disulfide analogs. ${ }^{45}$
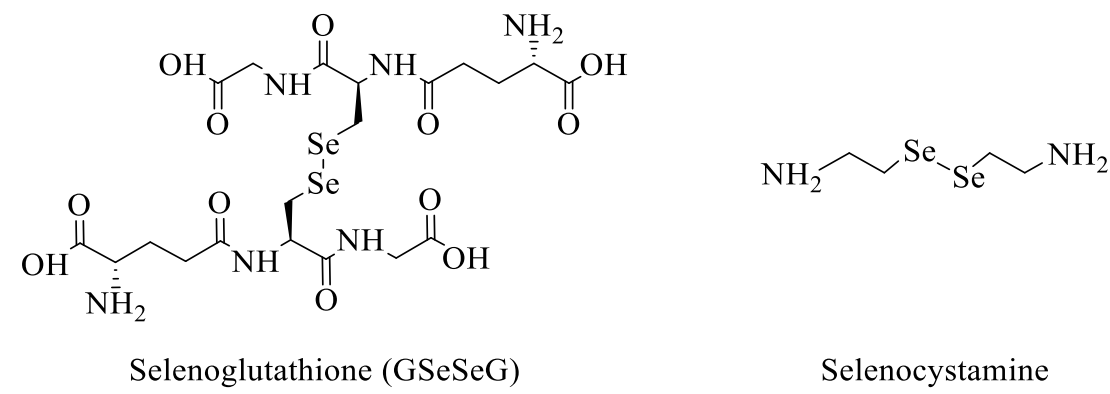

Figure 8 Structures of selenoglutathione and selenocystamine

\subsubsection{Aromatic thiols and disulfides}

Although PDI showed enhanced oxidative folding abilities in vitro, its application is limited owing to the high cost and the requirement of stoichiometric amounts for desirable catalytic activity. Water soluble aromatic thiols have thiol $\mathrm{pKa}$ values $(\mathrm{pKa}=4-7)$ close to the solvent-exposed thiol pKa value of PDI $(\mathrm{pKa}=6.7)$, and lower than those of aliphatic thiols ( $\mathrm{pKa}=7-11)$, such as GSH with a thiol $\mathrm{pKa}$ value of 8.7. The $p$-Substituted aromatic thiols and their corresponding disulfides are selected to fold reduced BPTI in vitro to minimize the steric hindrance. The thiolate ions of aromatic thiols with low thiol $\mathrm{pKa}$ values are more stable than aliphatic thiols, so the corresponding aromatic disulfides are more reactive than the corresponding aliphatic disulfides as the aromatic thiolate ions are better leaving groups in the thiol-disulfide interchange reaction. ${ }^{46}$ 
During oxidative protein folding, redox buffers containing aromatic thiols and disulfides can catalyze the formation and isomerization of protein disulfide bonds. The protein thiolate reacts with small molecule disulfide to form a mixed protein-small molecule disulfide, which is followed by the nucleophilic attack of another protein thiolate on the mixed disulfide to form a protein disulfide bond (Figure 9). Aromatic thiolate anions can help rearrange non-native disulfide to native disulfide bond through the formation of a mixed disulfide intermediate. Thus aromatic thiols and disulfides have been used to improve protein folding rates as they enhance the rates of the underlying thiol-disulfide interchange reactions in comparison to aliphatic thiols and disulfides. The folding rate of ribonuclease A was increased up to 23 -fold with aromatic thiols/disulfides versus the rate measured with GSH/GSSG. ${ }^{46}$ When folding lysozyme with aromatic thiols, the folding rate was increased by 11 -fold and the folding yield was increased $20 \%$ compared to the folding results with GSH/GSSG. ${ }^{47}$

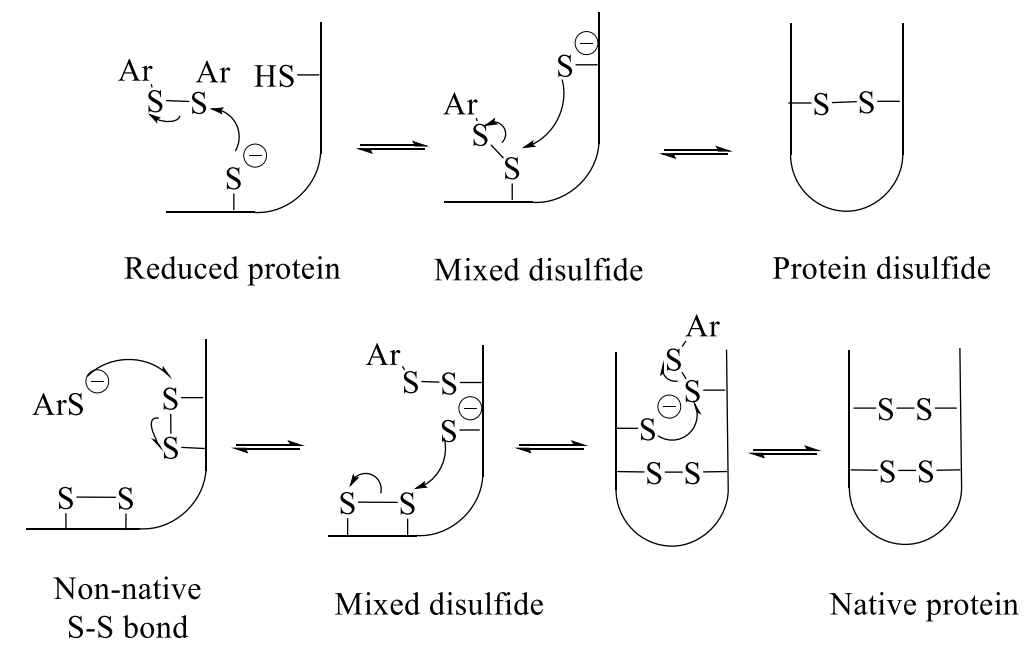

Figure 9 Thiol-disulfide interchanges of a protein with aromatic thiols and disulfides 


\subsection{In vitro oxidative folding of disulfide-containing proteins}

Oxidative folding of disulfide-containing proteins in vitro is always challenging because of the complicated folding process, involving multiple kinetically stable folding intermediates. With more than two disulfide bonds in the protein structure, protein folding can be slow and low yielding. ${ }^{48}$ Disulfide-containing proteins, including RNase A, lysozyme and BPTI, have been widely used as models to investigate the in vitro oxidative folding pathways because they are relatively small proteins with three or four native disulfide bonds in their native structures. These model proteins have been folded with various redox buffers under different $\mathrm{pH}$ and temperature. Folding intermediates with different types of disulfide bonds are trapped in the reactions, which can be used to analyze the protein disulfide bond formation through thiol-disulfide interchange reactions. Oxidative folding reactions can be quenched by alkylation or acidification. ${ }^{37}$ Commonly used alkylating reagents such as iodoacetate (IAA) and iodoacetamide (IAM) can rapidly block free thiol groups in a protein structure. Acidification is also used since the folding reaction can be rapidly quenched by lowering the $\mathrm{pH}$ through the addition of acid. The reversibility of the acid quenching process makes it possible to purify the folding intermediates at specific time points for further studies.

\subsubsection{Folding of ribonuclease A (RNase A)}

Bovine pancreatic ribonuclease A (RNase A) is one of the best-studied proteins for protein folding investigations, and was the model protein for Anfinsen's protein folding studies. $^{2}$ Ribonuclease A (RNase A) has 124 amino acid residues with four native disulfide bonds between Cys26-Cys40, Cys85-Cys95, Cys58-Cys110, and Cys65-Cys72 (Figure 10). 
The folding pathways of RNase A were characterized by the disulfide bonds found in the protein folding intermediates.

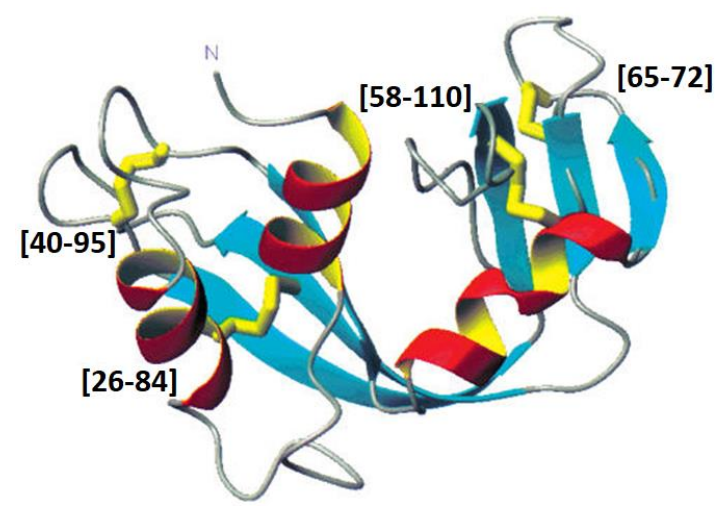

Figure 10 Structure of bovine pancreatic ribonuclease A (RNase A) (PDB: 7RSA)

Two folding stages were observed during the oxidative folding process of RNase A under typical folding conditions. ${ }^{23}$ At the early stage, also called the quasi-steady-state, reduced RNase A tends to fold rapidly in the presence of $\mathrm{DTT}^{\mathrm{ox}}$ to an unstructured ensemble of intermediates with one to four random disulfide linkages. For the folding ensembles, $\mathrm{nS}$ species represent the unstructured folding ensemble with $\mathrm{n}$ disulfide bonds that can interconverted rapidly with each other via thiol-disulfide interchange reactions. A preequilibrium was observed between reduced protein and unstructured $1 \mathrm{~S}-4 \mathrm{~S}$ folding species which do not have stable structures. The $4 \mathrm{~S}$ ensemble is a fully oxidized structure containing four disulfide bonds but without correct native structure. The unstructured $3 \mathrm{~S}$ ensembles are likely to reshuffle to structured three-disulfide bond containing folding species (3S* species) including des[40-95] and des[65-72] at the late stage. Des species describes the folding ensemble that has a native-like structure containing $n-1$ native disulfide bonds if there are $\mathrm{n}$ native disulfide bonds in the protein. ${ }^{49-50} \mathrm{~A}$ small amount of $2 \mathrm{~S}$ species $(<5 \%)$ can also refold to $3 \mathrm{~S}^{*}$ species via thiol-disulfide interchange reactions. ${ }^{51}$ 
Once $3 S^{*}$ species are formed, the three native disulfide bonds in either des[40-95] or des[65-72] are protected by the structure, but the remaining free thiols can be accessed by the solvent. Therefore, both $3 S^{*}$ species can be oxidized to the native form efficiently (Figure 11).

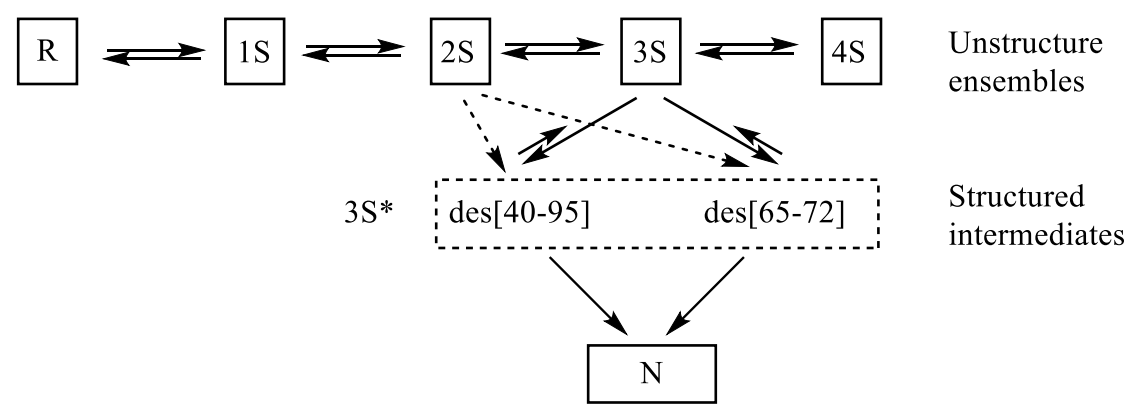

Figure 11 Oxidative folding pathway of RNase $\mathrm{A}^{49-50}$

Both reduced and scrambled RNase A were folded with aromatic thiols to improve the in vitro folding rates and yields. Scrambled RNase A is a fully oxidized form of the protein with non-native disulfide bonds. Aromatic thiols have been demonstrated to have enhanced nucleophilicity and better leaving group ability compared to aliphatic thiols such as GSH. When GSH was replaced with an aromatic thiol in the redox buffer, the folding rate of both forms of RNase A was increased about 6 times compared to the traditional folding conditions. The replacement of not only GSH with aromatic thiol but also GSSG with an aromatic disulfide did not enhance the folding rate further since aromatic disulfide can be rapidly formed during the folding with redox buffers containing GSSG/aromatic thiol. ${ }^{52}$ Folding of scrambled RNase A with aromatic thiols at different $\mathrm{pH}$ values showed that the best folding conditions were related to the concentration of active thiolate anions and not total thiol. The optimal folding concentration of total aromatic thiol decreased when the folding $\mathrm{pH}$ increased from 6.0-7.7. At $\mathrm{pH}$ 6.0, folding of scrambled RNase A showed up 
to 23 -fold faster folding rate with the optimal concentrations of aromatic thiols compared to GSH. Aromatic thiols having $\mathrm{pKa}$ values one unit lower than the folding $\mathrm{pH}$ were found to have better reactivity in protein folding reactions. ${ }^{46}$

Folding reaction of RNase A were also conducted in the presence of GSeSeG and the folding results were illustrated in terms of the catalytic activity to produce CMP from cCMP. ${ }^{42}$ Compared to GSSG, GSeSeG with the same concentration increased the folding rate of reduced RNase A by two-folds under aerobic folding conditions. When GSSG was replaced by GSeSeG, the folding rate and yield of RNase with the best conditions of GSSG and GSH pair can be maintained with only 10 times less concentration of GSeSeG. Since selenols have low pKa values, oxidative folding of RNase A can also be conducted effectively in acidic conditions. In comparison, the $\mathrm{pH}$ of traditional folding reactions was usually higher than 7 in order to keep GSH in its active thiolate form.

\subsubsection{Folding of lysozyme}

Hen egg white lysozyme (HEWL) is a glycosidase with 129 amino acid residues containing four native disulfide bonds at positions 6-127, 30-115, 64-80, and 76-94 (Figure 12).$^{53}$ Oxidative folding with lysozyme has been widely studied with different types of redox buffers and major folding intermediates have also been isolated and characterized. Lysozyme is an ideal protein to study protein folding as the yield of native lysozyme is very sensitive to the folding conditions with a range from $20 \%$ to $90 \%$ with different types and concentrations of the protein folding aids. ${ }^{54}$ Lysozyme may have non-productive aggregation during folding caused by nonspecific hydrophobic interactions. Some denaturants, such as guanidine hydrochloride $(\mathrm{GdnHCl})$ and L-arginine, can be added to the redox buffer. ${ }^{55}$ When the $\mathrm{GdnHCl}$ concentration in the folding buffers was increased, 
the folding yield of native lysozyme was increased but the folding rate decreased. Therefore, folding reactions of lysozyme needs to be conducted in the presence of optimal concentrations of $\mathrm{GdnHCl}$ that can minimize protein aggregation but yet provide desirable folding rates and yields.

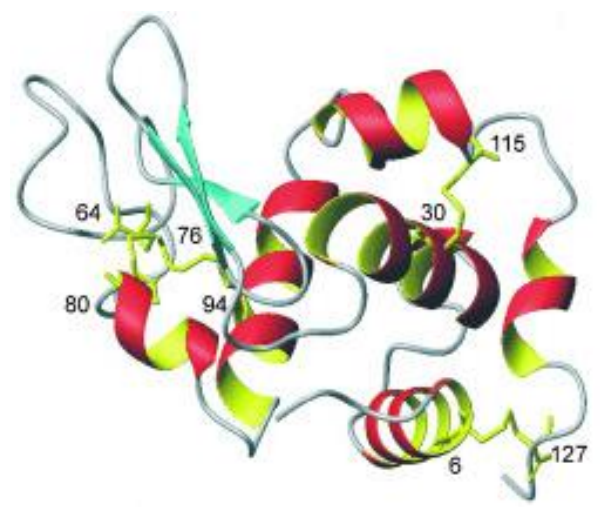

Figure 12 Structure of hen-egg-white lysozyme (PDB: 6LYZ)

The redox buffers that consisted of GSH and GSSG have been traditionally used to fold denatured-reduced lysozyme, and the optimal concentration ratio of GSH:GSSG to fold the protein was determined to be around 10:1. In the presence of $1 \mathrm{mM} \mathrm{GSH}$ and 0.2 $\mathrm{mM}$ GSSG and $2 \mathrm{M}$ urea, three kinetically stable three-disulfide intermediates were accumulated, des[76-94], des[6-127] and des[64-80], during the folding of lysozyme, which were determined to be native-like structures with three of the four native disulfide bonds. Among the three folding intermediates, des[76-94] accumulated to the highest percentage, and accounted for $40 \%$ of the total protein at one point (Figure 13). ${ }^{56}$ At the late stage of the folding reaction, des[76-94] was the only predominate intermediate, suggesting that the folding from the kinetic trap des[76-94] to native protein was the ratelimiting step during the folding of lysozyme. Since Cys94 in the des[76-94] structure was buried, des[76-94] needs to unfold and then form the disulfide bond between Cys76 and 
Cys 94 in order to obtain the native structure. The rearrangement of des[76-94] and native protein production were facilitated by adding urea and PDI as folding aids. Des[76-94] was reduced to a two-disulfide intermediates by PDI, and then re-oxidized to des[64-80] or des[6-127], which were folded to native protein rapidly.

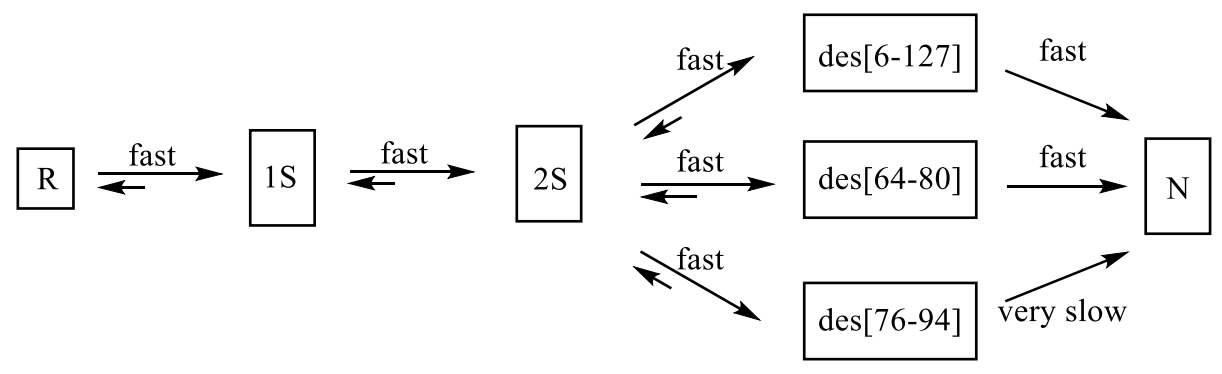

Native-like structure

Figure 13 Oxidative folding pathway of reduced lysozyme ${ }^{56}$

The redox buffers containing aromatic thiols and disulfides have been used to improve the folding rate and yield of reduced lysozyme. Aromatic thiols were expected to improve the folding rates owing to their better leaving group ability and better nucleophilicity compared to aliphatic thiols with similar thiol pKa values. When folding lysozyme with optimal concentrations of aromatic thiols, the folding rate was increased by 11 -fold and the folding yield was increased $20 \%$ compared to the folding results with GSH/GSSG. ${ }^{47}$

Folding of lysozyme with GSeSeG showed that a higher folding rate and yield of native lysozyme was obtained with GSeSeG and GSH compared to the folding with redox buffer with the same concentrations of GSSG and GSH. ${ }^{45}$ In addition, folding of higher concentrations of reduced lysozyme with GSeSeG shown higher folding rate and yield relative to GSSG. Folding of $140 \mu \mathrm{M}$ of lysozyme with GSeSeG/GSH obtained 50\% of native protein in $48 \mathrm{~h}$, while only $10 \%$ of native protein was produced with the same concentrations of GSSG/GSH. 


\subsection{In vitro oxidative folding of bovine pancreatic trypsin inhibitor (BPTI)}

Bovine pancreatic trypsin inhibitor (BPTI), also known as the protein based drug aprotinin, is a small globular protein that has been extensively studied for protein conformation, folding pathways, and protein/protein interactions ${ }^{57}$ In the structure of BPTI, there are 58 amino acid residues with three disulfide bonds between Cys 5-Cys 55, Cys 14Cys 38 and Cys 30-Cys 51, which help to stabilize the native form of the protein (Figure 14). The in vitro oxidative folding pathway of reduced BPTI has been characterized on the basis of disulfide bond formation and rearrangement of well-populated folding intermediates. Folding intermediates with one or two disulfide bonds have been identified and characterized in the folding of reduced BPTI with DTT ${ }^{\text {ox }}$ or GSH/GSSG.

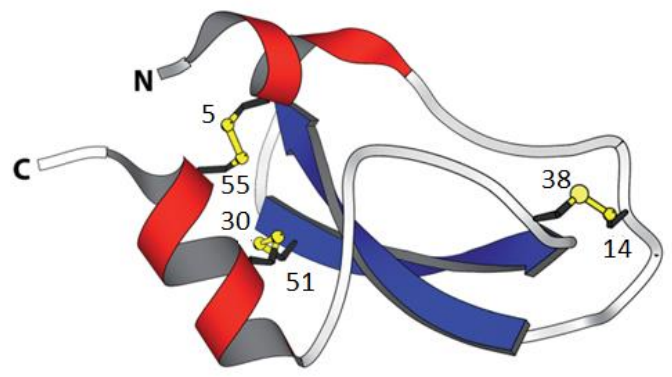

Figure 14 Structure of bovine panreatic trypsin inhibiotr (BPTI) (PDB: 1BPI)

\subsubsection{Folding of BPTI with traditional small molecule thiols and disulfides}

The folding pathway of reduced BPTI has been developed by Creighton's group in the presence of $\mathrm{DTT}^{\mathrm{ox}}$ at $\mathrm{pH}$ 8.7. Bovine pancreatic trypsin inhibitor (BPTI) contains six cysteine residues, which can form 74 possible folding intermediates with different numbers of protein disulfide bonds during folding. However, only several folding intermediates are predominant in the folding pathway of reduced BPTI (Figure 15). ${ }^{58-59}$ At the early stage of the folding process, the formation of one-disulfide folding intermediates is relatively 
random and rapidly reaches an equilibrium. The predominant one-disulfide intermediate [30-51] accumulates and can be further oxidized to two-disulfide folding intermediates. The second disulfide is formed between [14-38], [5-14], or [5-38] via intermolecular thioldisulfide interchange reactions with $\mathrm{DTT}^{\mathrm{ox}}$, so two-disulfide intermediates with non-native disulfide bonds are formed in Creighton's folding pathway. A native-like intermediate with two native disulfide bonds [5-55] and [14-38] can also be formed during folding. The twodisulfide intermediate [5-55, 14-38] has two remaining thiol groups buried in the hydrophobic core of the structure, making it difficult to oxidize to native protein through a direct oxidation pathway. Instead, disulfide bond rearrangement can occur to form a twodisulfide intermediate [30-51, 14-38], which can be further rearranged to another nativelike structure with two native disulfide bonds [30-51] and [5-55]. Since Cys14 and Cys 38 are both solvent accessible, $[30-51,5-55]$ can be further folded to native protein.

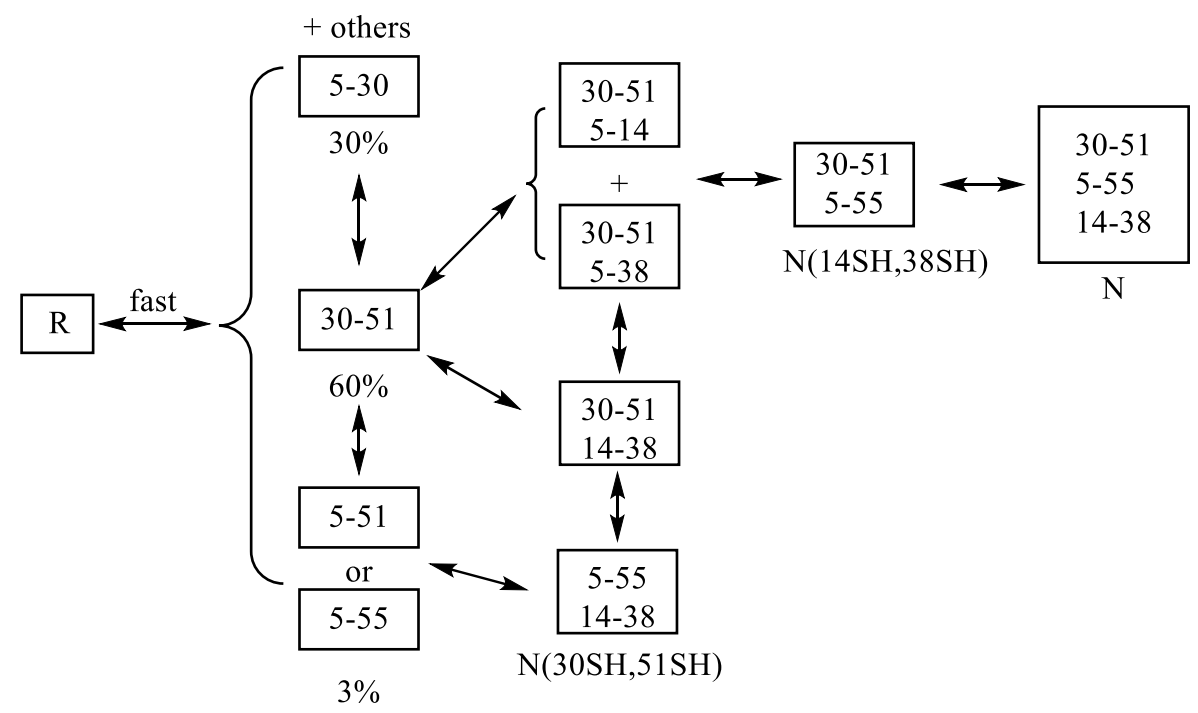

Figure 15 Oxidative folding pathway of reduced BPTI developed by Creighton's group ${ }^{58}$ Subsequently, the folding pathway of reduced BPTI was reexamined by Kim's group, showing that only folding intermediates with native disulfide bonds were accumulated 
during folding. ${ }^{37}$ The reactions were conducted under anaerobic conditions in the presence of GSSG at pH 7.3. Since the cysteine thiol has a pKa value close to 8.7, the net charge of folding intermediates can differ at the folding $\mathrm{pH}$ of 8.7. Therefore, folding of reduced BPTI at neutral pH 7.3 allowed simplification of the folding intermediate analysis. In addition, folding reactions were quenched by the addition of formic acid instead of alkylation by iodoacetic acid used in previous studies. The reversibility of the acid quenching process makes it possible to obtain the folding intermediates formed at specific time points and then to refold then again at more basic $\mathrm{pH}$. Therefore, acid quenching was used and the folding intermediates were purified by RP-HPLC.

The folding pathway of reduced BPTI with GSSG was proposed on the basis of the formation and rearrangement of disulfide bonds, which is called the rearrangement type pathway (Figure 16). ${ }^{37}$ Two one-disulfide intermediates, [5-55] and [30-51], were accumulated via rapid thiol-disulfide interchange reactions from reduced BPTI. Then two predominant two-disulfide intermediates were formed by the oxidation of the two cysteines thiols between Cys14 and Cys38, which were entitled as $N^{*}([5-55 ; 14-38])$ and $N^{\prime}$ ([14$38 ; 30-51])$. Since two free thiols, in both $\mathrm{N}^{\prime}$ and $\mathrm{N}^{*}$, were buried in the hydrophobic core of the structure, the rearrangement rate to native structure was slow, which made N' and $\mathrm{N}^{*}$ kinetic traps. Both $\mathrm{N}^{\prime}$ and $\mathrm{N}^{*}$ had to undergo intramolecular disulfide bond rearrangement in order to reach the native state. Once $\mathrm{N}^{\prime}$ and $\mathrm{N}^{*}$ were rearranged to another two-disulfide intermediate $\mathrm{N}^{\mathrm{SH}}$, the native protein was produced with the rapid formation of the third disulfide bond [14-38] in $\mathrm{N}^{\mathrm{SH}}$. 


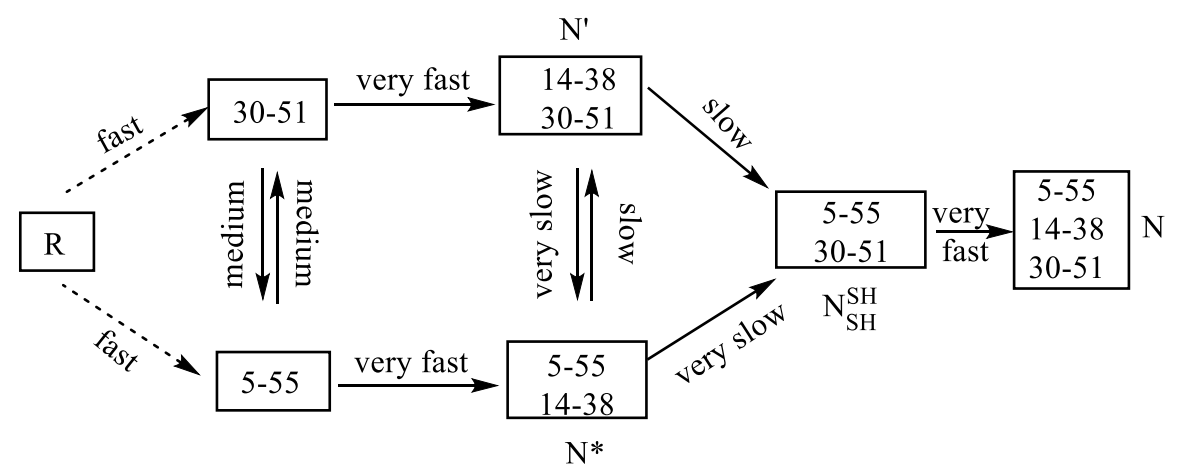

Figure 16 Oxidative folding pathway of reduced BPTI developed by Kim's group ${ }^{37}$

It has been demonstrated that a new one-disulfide intermediate [14-38] was observed at the early stage of reduced BPTI folding with GSSG. ${ }^{60}$ However, [14-38] was less thermodynamically stable than the other well-populated one-disulfide intermediates [3051] and [5-55], so it rapidly rearranged to [30-51] or [5-55]. The transient level of onedisulfide intermediate [14-38] was shown to be related to the formation rate of mixed disulfide bonds of GSSG and either Cys14 or Cys38. During folding with higher concentration of GSSG, more [14-38] was formed at the early folding stage. However, the maximal formation level of one-disulfide intermediates [30-51] and [5-55] was not affected by high concentration of GSSG. Interestingly, the disulfide bond [14-38] was also the last one formed in the native structure, as shown in the folding pathway of reduced BPTI.

The two-disulfide intermediate $\mathrm{N}^{\prime}$ was further investigated under different concentrations of GSSG. ${ }^{61}$ With moderate concentrations of GSSG, the two remaining free thiols in $\mathrm{N}^{\prime}$ were buried in the hydrophobic core and not accessible to GSSG, so N' was rearranged to $\mathrm{N}^{\mathrm{SH}}$ instead of direct oxidation of the free thiols with GSSG. Although the rate of $\mathrm{N}^{\mathrm{SH}}$ disulfide bond rearrangement was slow, it was still 150 times faster than the rate of free thiols in $\mathrm{N}^{\prime}$ reacting with GSSG directly. When the folding was conducted under 
high GSSG concentrations, $\mathrm{N}^{\prime}$ reacted with GSSG to form mixed disulfides $\mathrm{N}^{\prime}(\mathrm{SG})$ between either Cys5 or Cys 55 in the protein and GSSG including N'[5-SSG] and N'[55SSG]. Singly mixed disulfide $\mathrm{N}^{\prime}(\mathrm{SG})$ was rapidly oxidized to a doubly mixed disulfide intermediate $\mathrm{N}^{\prime}$ [5-SSG; 55-SSG] with no free thiol groups, which became a nonproductive dead-end folding intermediate in the folding process. Therefore, folding with only high concentrations of oxidizing agents such as GSSG $(0.25 \mathrm{mM})$ was not efficient through the formation of mixed disulfide intermediates via growth type pathway (Figure 17).

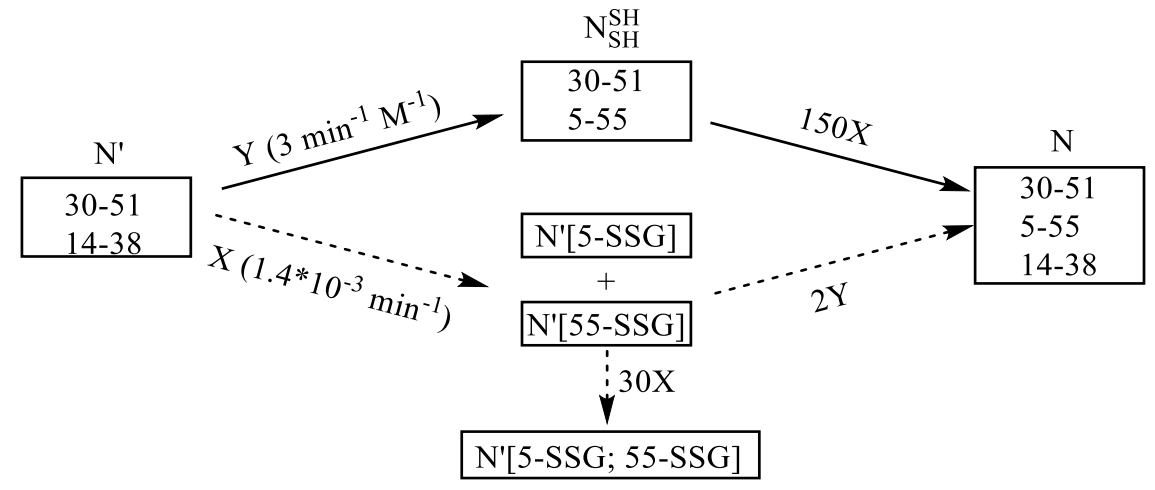

Figure 17 Oxidative folding of N' via growth type and rearragment type pathway ${ }^{61}$

The difference between Creighton's and Kim's folding pathways of reduced BPTI is the accumulation of nonnative disulfide intermediates. In Creighton's folding pathway, nonnative disulfide folding intermediates were kinetically stable and well-populated during folding. ${ }^{62}$ Creighton et al. stated that formation of folding intermediates with only native disulfide bonds in Kim's folding pathway was incorrect, since the amount of the folding intermediates did not equate with the kinetic importance of the intermediates. In addition, the disulfide bond formation between Cys14 and Cys 38 was essential to form twodisulfide intermediates, $\mathrm{N}^{\prime}$ and $\mathrm{N}^{*}$, in Kim's pathway. If Cys 14 or Cys 38 was blocked, the folding process could be stopped at the early folding stage. However, it has been shown 
that the folding of the protein with blocked Cys14 or Cys38 could still be folded to nativelike structure $\mathrm{N}^{\mathrm{SH}}$ with two disulfide bonds [30-51] and [5-55]. ${ }^{63}$

In response, Kim claimed that only folding intermediates containing native disulfide bonds were accumulated at both folding $\mathrm{pHs}$ of 7.3 and 8.7, the latter of which was identical to the $\mathrm{pH}$ in Creighton's folding experiments. ${ }^{64}$ Folding to $\mathrm{N}^{\prime}$ and $\mathrm{N}^{*}$ was the preferred folding pathways of reduced BPTI, so there were alternate routes if Cys14 and Cys 38 residues were blocked. The folding rate of any intermediates II to $\mathrm{N}^{\mathrm{SH}}$ was almost the same when folding with wild-type reduced BPTI and mutant BPTI with blocked Cys14 or Cys $38 .{ }^{65}$ The folding pathway could be simplified by the formation of any types of one-

disulfide intermediate I and two-disulfide intermediates II other than $\mathrm{N}^{\mathrm{SH}}$. Therefore, Kim's group confirmed folding intermediates with native disulfide bonds were predominant in the folding pathway of reduced BPTI.

\subsubsection{Folding of BPTI with selenoglutathione (GSeSeG)}

It has been confirmed that GSeSeG possessed increased catalytic efficiency on oxidative folding of reduced BPTI under both aerobic and anaerobic conditions. ${ }^{42}$ The folding rates and yields of native BPTI were improved in the presence of GSeSeG relative to GSSG. Under anaerobic conditions, the folding of reduced BPTI with $150 \mu \mathrm{M} \mathrm{GSeSeG}$ showed a fast folding rate. The accumulation of major folding intermediates was observed within $1 \mathrm{~min}$. The folding process was completed in $24 \mathrm{~h}$. In comparison, folding of reduced BPTI with the same concentration of GSSG was relatively slow and only formed 50\% of native protein after $24 \mathrm{~h}$. The effect of GSeSeG was even more striking when folding reactions of reduced BPTI were conducted aerobically. In the presence of atmospheric 
oxygen, selenols can be reoxidized back to diselenide, which can be further used to fold the protein to the native form. The folding rate of reduced BPTI was increased significantly.

Two kinetically stable folding intermediates $\mathrm{N}^{\prime}$ and $\mathrm{N}^{*}$ were also folded individually by the addition of different concentrations of GSeSeG. As shown in Kim's folding pathway of reduced BPTI, $\mathrm{N}^{\prime}$ and $\mathrm{N}^{*}$ have native-like structures and the two remaining thiol groups are buried in the hydrophobic core of the structure, which are considered as kinetic traps. Folding of N' with GSeSeG showed different folding intermediate distributions compared to the folding with GSSG. The accumulation of the two-disulfide intermediate $\mathrm{N}^{\mathrm{SH}}$ was caused by the fast reaction between $\mathrm{N}^{\mathrm{SH}}$ and GSeSeG. A mixed selenosulfide folding intermediate $\mathrm{N}^{\prime}(\mathrm{SeG})$ was accumulated at the early folding stage. When increasing the concentration of $\mathrm{GSeSeG}$, more $\mathrm{N}^{\prime}(\mathrm{SeG})$ and other nonproductive folding intermediates were accumulated and remained at high levels throughout the folding process. These intermediates became kinetic traps. Therefore, GSeSeG only showed a moderate increase in the folding rate of $\mathrm{N}^{\prime}$ compared to GSSG.

Since GSeSeG is more reactive than GSSG, $\mathrm{N}^{*}$ was folded to a singly mixed selenosulfide intermediate $\mathrm{N}^{*}(\mathrm{SeG})$ through direct oxidation. $\mathrm{N}(\mathrm{SeG})$ was then attacked by the other free thiol in the protein through intramolecular selenol-disulfide interchanges, leading to the formation of native protein. A doubly mixed selenosulfide intermediate $\mathrm{N}^{*}(\mathrm{SeG})_{2}$ was observed at higher $\mathrm{GSeSeG}$ concentrations. Owing to the lack of free thiols in the protein structure, the formation of $\mathrm{N}^{*}(\mathrm{SeG})_{2}$ was nonproductive and delayed the folding process. Nonetheless, folding of $\mathrm{N}^{*}$ to native protein using optimal concentrations of GSeSeG showed higher folding rates than with GSSG that were comparable to the folding rate of N' to native protein. GSeSeG/GSeH could also be used to rescue the kinetic 
traps that were observed during the folding of disulfide-containing proteins with traditional redox buffers containing GSSG/GSH.

Two selenoproteins, C5U BPTI and C5U/C38U BPTI, were prepared by replacing Cys5 and Cys14/Cys38 in wild type BPTI, respectively. ${ }^{44}$ The folding of C5U BPTI analog was completed in $3 \mathrm{~h}$ and more than $50 \%$ of the native protein formed within $1 \mathrm{~min}$. The free selenol group in C5U N' promoted the thiol/disulfide interchange, so C5U N' did not accumulate and rapidly rearranged to $\mathrm{C} 5 \mathrm{U} \mathrm{N}_{\mathrm{SH}}$ and ultimately the native structure. During the folding of the $\mathrm{C} 14 \mathrm{U} / \mathrm{C} 38 \mathrm{U}$ BPTI analog, the rate of thiol-disulfide interchange reactions was increased by the presence of a diselenide bond in the protein structure. The folding of BPTI analogs with selenocysteine substitutions showed a folding pathway that was similar to the wild type BPTI but with higher folding rates and yields.

\subsection{Experimental methods used in protein folding analysis}

Protein folding/unfolding is known as a complicated process since many folding intermediates can be accumulated which need to be characterized in order to elucidate the folding pathways of the target protein. A number of analytical techniques have been used to monitor protein conformational changes during the folding and unfolding process. ${ }^{66}$

\subsubsection{Hydrogen/deuterium exchange (HDX)}

The structural dynamics of the protein has been determined by hydrogen/deuterium exchange (HDX) during the protein folding process. ${ }^{67}$ The HDX process was conducted by exposing a protein to $\mathrm{D}_{2} \mathrm{O}$ solvent during folding, so that some of the hydrogen atoms in the protein structure were exchanged with the deuterium atom rapidly and the deuteron was incorporated into the protein structure. The HDX exchange reactions were most likely to occur within less ordered regions in the protein structure, where the hydrogen atoms are 
exposed to the $\mathrm{D}_{2} \mathrm{O}$ solvent and lacked hydrogen bonding interactions. ${ }^{68}$ The $\mathrm{H}$ to $\mathrm{D}$ exchange rates are affected by protein conformational change, so the measurement of protein deuteration change can help better understand how protein folds and unfolds over time. Usually hydrogens in the peptide backbone amide linkages (NHs) are deuterated and it is advantageous to monitor the HDX of amide hydrogen since almost all amino acids (except proline) contain an amide hydrogen. As a result, the secondary structures of the protein, such as $\alpha$-helices and $\beta$-sheets, are closely related to the hydrogen bonding interactions formed by amide hydrogens.

Many methods have been used to monitor the HDX process, such as nuclear magnetic resonance (NMR) spectroscopy and mass spectrometry (MS). Due to the magnetic property difference, hydrogen and deuterium can be distinguished using NMR spectroscopy ${ }^{69}$ BPTI and its homologous proteins were shown to be ideal models to study structural changes using HDX-NMR methods as they contain two hydrogen-bonded $\alpha$-helices and a small $\beta$ sheet in the protein structure. ${ }^{70-71}$ Since the mass of deuterium was different from that of hydrogen, HDX-MS is also an important way to detect protein conformational changes and dynamics. Continuous labeling of the protein sample with deuterium from the $\mathrm{D}_{2} \mathrm{O}$ solvent was conducted. Next the reaction was quenched with an acid at specific time points and the quenched protein samples were then analyzed by MS to determine protein structural information on the basis of changes in the number of deuteration sites in the protein. ${ }^{72}$ The advantages of HDX-MS are that only a small amount of protein is required and samples with multiple proteins can also be analyzed when liquid chromatography (LC) separation of the protein mixture is carried out prior to MS analysis. ${ }^{73}$ 


\subsubsection{Chromatographic methods}

Size exclusion chromatography (SEC) is known as a useful tool to remove small molecule denaturants and separate folding intermediates in protein folding and unfolding analysis. ${ }^{74}$ On-column protein folding of RNase A has been successfully conducted using gel filtration chromatography. RNase A was refolded on a Sephacryl column and the recovery of native RNase A was above $90 \%$ relative to the denatured protein. Improvements of protein folding analysis with gel filtration have been achieved by using a concentration gradient of denaturants and a $\mathrm{pH}$ gradient of the elution buffer. ${ }^{75} \mathrm{~A}$ singlechain variable fragment $(\mathrm{scFv})$ fusion protein was refolded on a Superdex 30 prep-grade column with a urea and a $\mathrm{pH}$ gradient. The protein sample with the higher molecular weight was eluted faster from the column than urea and buffer salts. As the protein was eluted into the refolding buffer though the different $\mathrm{pH}$ and urea concentration, folded $\mathrm{scFv}$ fragments were produced and the yield was improved compared to the folding without gradient elution. $^{76}$

Ion exchange chromatography (IEC) was first used by Creighton in protein folding analysis. ${ }^{77}$ Folding reactions of reduced BPTI were conducted with GSSG and quenched with iodoacetate at certain time points. Iodoacetate would alkylate free thiols in the protein structure and introduced an additional negative charge to the protein folding intermediates, making it possible to separate the trapped intermediates at each time point using the IEC method. Since the proteins were positively charged at the folding $\mathrm{pH}$, cation exchange chromatography was conducted using CM-cellulose as the stationary phase. A concentration gradient of $\mathrm{NaCl}$ was used in the elution buffer, so the folding intermediates with various net charges was separated by chromatography. The dual gradient elution 
method of changing urea concentration and $\mathrm{pH}$ has also been used in IEC analysis in order to improve the protein folding yield. ${ }^{78}$ When reduced lysozyme was folded by dual gradient IEC on a SP Sepharose Fast Flow column, The activity of native lysozyme to lyse suspensions of Micrococcus lysodeikticus was more than 95\% and increased by $25 \%$ compared to the IEC folding with gradient elution.

Reversed phase-high performance liquid chromatography (RP-HPLC) has been commonly used for in vitro oxidative protein folding analysis, as RP-HPLC efficiently allows the separation of native protein and folding intermediates formed during the folding process. ${ }^{37}$ The folding analysis of reduced BPTI showed that HPLC was a more accurate and sensitive method to separate folding intermediates trapped at specific time point comparted to the IEC analysis. More protein peaks were obtained on HPLC chromatograms when the folding intermediates collected by IEC were analyzed by HPLC. In order to develop the oxidative folding pathway of reduced BPTI, the folding intermediates formed during the folding process were purified by using HPLC on a semipreparative C18 column and further oxidized to native protein.

\subsubsection{Capillary electrophoresis (CE)}

Protein folding and unfolding kinetics have been accurately monitored by capillary zone electrophoresis (CZE), which directly determines the appearance and disappearance of intermediates during the folding/unfolding process.$^{79}$ The thermodynamic parameters of the lysozyme folding process, such as enthalpy change $(\Delta \mathrm{H})$ and entropy change $(\Delta \mathrm{S})$, were quantitatively characterized when free solution CE was applied to the folding analysis, which showed the relationship between the mobility and the changes of protein charge and composition. ${ }^{80}$ 
Electrospray ionization mass spectrometry (ESI MS) provides a sensitive method to analyze protein folding intermediates by determining the molecular weight of intermediates formed during the unfolding/folding process. Disulfide-containing proteins tend to have different charge state distributions with a change in the folding conditions. Proteins unfold further with the reduction of disulfide bonds and this reduction allows more acidic/basic amino acid residues to be exposed for protonation/deprotonation, so the folding intermediates with different disulfide bond formation can be characterized by different charge states. ${ }^{81}$ On-line capillary isoelectric focusing-electrospray ionization mass spectrometry (CIEF-ESI MS) was also used to monitor the oxidative folding process of reduced RNase A with redox buffers containing GSH and GSSG. ${ }^{82}$ Folding reactions were quenched by alkylating with iodoacetate (IAA) the free thiol groups of the folding intermediates at specific time points. Since the introduction of IAA to the folding intermediate structure added a -1 charge and a molar mass of 58 at each alkylation site, folding intermediates with different numbers of disulfide bonds were separated by the $\mathrm{pI}$ difference, and directly characterized by ESI MS according to their molecular mass difference. 


\section{Objectives}

The overall aim of the present project is to improve the oxidative folding of reduced BPTI with aromatic thiols and their corresponding disulfides in vitro compared to traditional aliphatic small molecules such as GSH and GSSG.

\subsection{Investigation of the thiol pKa and charge effects of aromatic thiols on the folding of reduced BPTI.}

The pKa values of small molecule thiols have been shown to be correlated to the folding reaction rates of disulfide-containing proteins. BPTI with an isoelectric point (pI) close to 10.5 is positively charged at the folding solution $\mathrm{pH}$ of 7.3 . The charges of small molecules affect the formation of native protein and the folding intermediates. Therefore, aromatic thiols with varying thiol pKa values can be charges are used to fold reduced BPTI in vitro.

\subsection{Oxidative folding analysis of reduced BPTI using aromatic thiols and disulfides with different hydrophobicity.}

Aromatic thiols with an elongated alkyl group on the aromatic ring are expected to increase interactions with the hydrophobic core of disulfide-containing proteins s during folding, allowing more facile access to buried disulfide bonds. The folding rate of reduced BPTI can be improved in the presence of redox buffers containing aromatic thiols and disulfides with long hydrophobic chains.

\subsection{Determination of the oxidative folding kinetics of reduced BPTI with small molecule thiols and disulfides using capillary electrophoresis.}

Capillary electrophoresis (CE) has great potential for the analysis of macromolecule kinetics, due to its low volume sample requirement (sub $\mu \mathrm{L}$ ), high resolution and lack of 
stationary phase interaction. Capillary electrophoresis has been used to monitor protein folding and unfolding. Hence CE should be a useful technique to study the folding of reduced BPTI with small molecule thiols and disulfides. 


\section{Investigation of charge effects of aromatic thiols on reduced BPTI folding}

\subsection{Abstract}

Oxidative folding of disulfide-containing proteins, such as lysozyme and ribonuclease A, has confirmed that aromatic thiols and disulfides can increase both the folding yield and rate compared to traditional redox buffers. In the present study, three aromatic thiols, including sulfonic acid thiol (SA), phosphoric acid thiol (PA), and quaternary ammonium salt thiol (QAS), and their corresponding disulfides with different charges were used to folding reduced BPTI in vitro. Reduced BPTI was folded with varying concentrations of each aromatic thiol and its disulfide at $\mathrm{pH} 7.3$ to determine the best folding conditions. Redox buffers containing aromatic thiols and disulfides significantly increased the folding rate of reduced BPTI compared to the traditional redox buffer containing GSH and GSSG. With the best concentrations of positively charged QAS thiol and disulfide, almost $90 \%$ of native BPTI was produced within $2 \mathrm{~h}$. In comparison, after $2 \mathrm{~h}$ only $25 \%$ of native protein was produced when folding with the best concentrations of GSH and GSSG, and it required $2 \mathrm{~d}$ to obtain $90 \%$ native protein. Protein precipitation occurred during the folding with SA and PA thiols presumably caused by the aggregation of mixed disulfide folding intermediates formed by positively charged protein and negatively charged SA or PA thiol.

\subsection{Introduction}

Aromatic thiols are small molecules that were designed by mimicking the physical properties of the active site of protein disulfide isomerase (PDI), which is an enzyme that catalyzes the folding of disulfide-containing proteins in eukaryotes. During oxidative protein folding, a protein thiolate reacts with a small molecule disulfide to form a mixed disulfide bond, which is followed by the nucleophilic attack of another protein thiolate on 
the mixed disulfide to form a native disulfide bond. Compare to GSH ( $\mathrm{pKa}=8.7$ ), aromatic thiols have low pKa values between 5 to 7 , which are also lower than the folding solution $\mathrm{pH}$ of 7.3. The reaction rate of small molecule thiols is related to the concentration of deprotonated thiolate anions, and a large proportion of the aromatic thiols is in the active thiolate form at $\mathrm{pH}$ 7.3. In comparison, most of GSH is in the thiol form at $\mathrm{pH} 7.3$, which is unreactive in thiol-disulfide interchange reactions. Thus aromatic thiols and disulfides are expected to improve protein folding as they enhance the rates of the underlying thioldisulfide interchange reactions compared to aliphatic thiols and disulfides such as GSH/GSSG.

Aromatic thiols and disulfides increased both the folding yield and rate of disulfidecontaining proteins compared to traditional redox buffers, such as GSH/GSSG. The folding rate of RNase A was increased up to 23-fold with aromatic thiols and disulfides versus the rate measured with GSH/GSSG. ${ }^{46}$ When folding lysozyme with aromatic thiols, the folding rate was increased by 11 -fold and the folding yield in terms of the native protein activity was increased $20 \%$ compared to the folding results with GSH/GSSG. ${ }^{47}$ However, the folding of reduced BPTI with aromatic thiols and disulfides has not been studied.

Folding of reduced BPTI with aliphatic thiols and disulfide has been widely investigated in previous studies, but the process is always slow and low yielding. Three aromatic thiols, including SA, PA and QAS, and their corresponding disulfides were used to fold reduced BPTI in vitro (Figure 18). All three aromatic thiols have low thiol pKa values and are $p$-substituted which minimizes steric hindrance. Bovine pancreatic trypsin inhibitor (BPTI) has a pI of 10.5 and thus is positively charged at the folding $\mathrm{pH}$ of 7.3. Aromatic thiols SA and PA are negatively charged and QAS is positively charged, which 
can lead to charge effects on the formation of folding intermediates containing disulfide bonds between proteins and small molecule aromatic thiols. In addition, the best conditions of each aromatic thiol and its corresponding disulfide to folding reduced BPTI were determined and the results were compared with that of folding with redox buffers containing GSH and GSSG.

$\mathrm{SA}, \mathrm{pKa}=5.7$

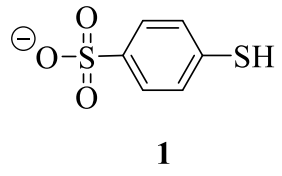

$\mathrm{PA}, \mathrm{pKa}=6.6$

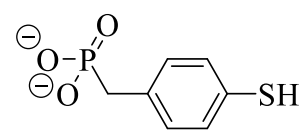

2

QAS, $\mathrm{pKa}=5.5$

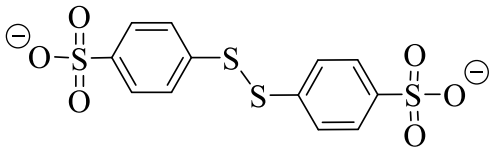

4

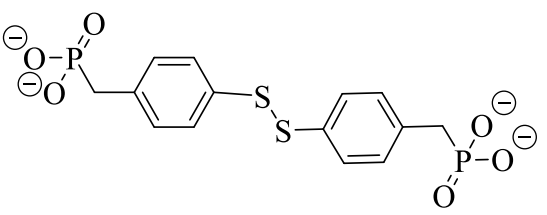

5

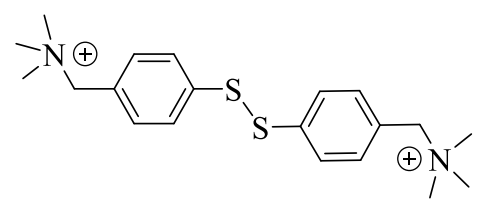

6

Figure 18 Aromatic thiols SA, PA and QAS and their corresponding disulfides

\subsection{Experimental section}

\subsubsection{Materials}

Native BPTI, Trisma base, bis-tris propane, guanidine hydrochloride ( $\mathrm{GdnHCl})$, potassium choride $(\mathrm{KCl})$, dithiothreitol (DTT), ethylenediaminetetraacetic acid (EDTA), reduced glutathione (GSH), glutathione disulfide (GSSG) were purchased from Sigma Aldrich. Sephadex ${ }^{\mathrm{TM}}$ G-25 Fine was purchased from GE Healthcare. Trifloroacetic acid (TFA), acetonitrile (ACN) and concentrated hydrochloric acid $(\mathrm{HCl})$ were purchased from Fisher Scientific. Nanopure deionized water was generated from a Barnstead water system. All aromatic thiols and disulfides for protein folding reactions were synthesized and 
purified in the lab. The absorbance and UV spectra were determined with a Cary 300 spectrophotometer. The $\mathrm{pH}$ was measured with a VWR symphony SB20 $\mathrm{pH}$ meter.

All RP-HPLC analysis was performed on a Hitachi D-7000 system equipped with a L-7400 UV-Vis detector. Three C18 columns including Alltech Macrosphere C18 preparative column $(250 \times 22 \mathrm{~mm})$, Vydac C18 semi-preparative column $(250 \times 10 \mathrm{~mm})$ and Vydac C18 analytical column $(250 \times 4.6 \mathrm{~mm})$ were used for different purposes.

\subsubsection{Preparation of reduced BPTI}

Tris buffer was prepared by dissolving $1.213 \mathrm{~g}$ Trizma base $(0.01 \mathrm{~mol}), 1.491 \mathrm{~g} \mathrm{KCl}$ $(0.02 \mathrm{~mol})$ and $0.0373 \mathrm{~g}$ EDTA $(0.10 \mathrm{mmol})$ in $80 \mathrm{~mL}$ deionized water. The $\mathrm{pH}$ of the solution was adjusted to 8.7 with concentrated $\mathrm{HCl}(12.1 \mathrm{~N})$ and the volume adjusted to $100 \mathrm{~mL}$ by the addition of deionized water. The final Tris buffer contained $0.10 \mathrm{M}$ Trizma, $0.20 \mathrm{M} \mathrm{KCl}$ and $1.0 \mathrm{mM}$ EDTA. The reduction mixture was prepared by dissolving 5.731 $\mathrm{g} \mathrm{GdnHCl}(0.06 \mathrm{~mol})$ and $0.0771 \mathrm{~g}$ DTT $(0.5 \mathrm{mmol})$ with Tris buffer in a $25^{\circ} \mathrm{C}$ water bath. The final volume of the reduction mixture was $10 \mathrm{~mL}$ and the final concentration was $6 \mathrm{M}$ GdnHCl and 0.05 M DTT.

In order to unfold the protein, Native BPTI was added to the reduction mixture to get the final concentration of $6.5 \mathrm{mg} / \mathrm{mL}$. The resulting solution was kept in a water bath at 25 ${ }^{\circ} \mathrm{C}$ for $1 \mathrm{~h}$, followed by the addition of $0.2 \mathrm{~N} \mathrm{HCl}$ to adjust the $\mathrm{pH}$ of the solution to between 2 and 3 . Then the protein was purified by gel filtration on a Sephadex G- 25 column using $0.01 \mathrm{~N} \mathrm{HCl}$ as the mobile phase. The protein content of collected factions was determined by measuring the absorbance at $280 \mathrm{~nm}$ with a UV-Vis spectrophotometer. The reduced protein has an extinction coefficient of $5377 \mathrm{~cm}^{-1} \mathrm{M}^{-1}$ at $280 \mathrm{~nm} .{ }^{38}$ All fractions containing reduced protein were combined and lyophilized. The lyophilized protein was dissolved in 
$0.01 \mathrm{~N} \mathrm{HCl}$ and purified by RP-HPLC on a C18 preparative column. The mobile phase was a mixture of solvent A with $0.1 \%$ TFA in water and solvent B with $90 \%$ acetonitrile with $0.1 \%$ TFA in water. The flow rate was $5 \mathrm{~mL} / \mathrm{min}$ and following linear elution gradient was used: 0 min, $90 \%$ buffer A; 20 min, $65 \%$ buffer A; 100 min $61 \%$ buffer A, 120 min,

$50 \%$ buffer A. The absorbance for reduced BPTI preparation was monitored at $280 \mathrm{~nm}$. All fractions containing reduced BPTI were collected. The purity of the collected protein samples was analyzed on a Vydac C18 analytical column. The absorbance was monitored at $229 \mathrm{~nm}$ with a flow rate of $1 \mathrm{~mL} / \mathrm{min}$. The elution gradient for analyzing each fraction was: 0 min, $90 \%$ solvent $\mathrm{A} ; 15 \mathrm{~min}, 73 \%$ solvent $\mathrm{A} ; 35 \mathrm{~min}, 71 \%$ solvent $\mathrm{A}, 50 \mathrm{~min}, 69 \%$ solvent A; $70 \mathrm{~min}, 65 \%$ solvent A. The pure reduced BPTI was lyophilized and then dissolved in $0.01 \mathrm{~N} \mathrm{HCl}$ solution.

\subsubsection{Preparation of SA, PA and QAS thiols and disulfides}

Three aromatic thiols with different $p$-substituents, PA, SA and QAS thiols, were synthesized in the lab following previous literature pocedures. ${ }^{47,83}$ The corresponding disulfides were prepared by letting the thiol solutions stir in air until the thiols were completely oxidized to their corresponding disulfides. Then aromatic thiols and disulfides were purified by RP-HPLC using a Vydac C18 semi-preparative column. Each time $2 \mathrm{~mL}$ of aromatic thiol or disulfide sample was injected on to an HPLC column manually. The column was heated to $50{ }^{\circ} \mathrm{C}$ and the flow rate was $3 \mathrm{~mL} / \mathrm{min}$. The mobile phase was a mixture of solvent A ( $0.1 \%$ TFA in water) and solvent B (90\% acetonitrile with $0.1 \%$ TFA in water). The following elution gradient was used: 0 min, $90 \%$ solvent A; 4 min, 85\%, 20 min, $75 \%$ solvent $\mathrm{A} ; 30 \mathrm{~min}, 60 \%$ solvent $\mathrm{A} ; 50 \mathrm{~min}, 55 \%$ solvent $\mathrm{A}, 70 \mathrm{~min}, 50 \%$ solvent A. The absorbance was monitored at $252 \mathrm{~nm}$. The collected fractions were analyzed by 
RP-HPLC on a C18 analytical column. All fractions containing only aromatic thiol or aromatic disulfide were combined. All thiol and disulfide samples were lyophilized, dissolved in deoxygenated water, and kept at $-20{ }^{\circ} \mathrm{C}$ prior to folding studies.

\subsubsection{Folding of reduced BPTI with aromatic thiols and disulfides}

Reduced BPTI was folded with different concentrations and combinations of aromatic thiols and their corresponding disulfides in refolding buffer at $\mathrm{pH} 7.3$. The $1.5 \times$ refolding buffer was prepared by mixing bis-tris propane ( $15 \mathrm{mmol}, 4.23 \mathrm{~g}), \mathrm{KCl}(30 \mathrm{mmol}, 2.24 \mathrm{~g})$, EDTA $(0.15 \mathrm{mmol}, 0.056 \mathrm{~g})$ in $80 \mathrm{~mL}$ of deionized water. The $\mathrm{pH}$ of the buffer solution was adjusted to 7.3 with concentrated $\mathrm{HCl}$ and the solution was made to $100 \mathrm{~mL}$ by the addition of deionized water. The buffer was then deoxygenated by passing argon through it for $30 \mathrm{~min}$. Reduced BPTI was diluted to a final concentration of $30 \mu \mathrm{M}$ by the addition of the refolding buffer. The final refolding buffer contained $0.10 \mathrm{M}$ bis-tris propane, 0.20 $\mathrm{M} \mathrm{KCl}$ and $1.0 \mathrm{mM}$ EDTA.

The redox buffers consisting of each aromatic thiol and its corresponding disulfide were used to fold reduced BPTI in vitro. Folding reactions were conducted at $\mathrm{pH} 7.3$ in a $25{ }^{\circ} \mathrm{C}$ water bath under argon. At certain refolding time points, $300 \mu \mathrm{L}$ aliquots of the reaction mixture were removed and quenched with formic acid. Acid quenched samples were stored on an ice bath immediately prior to HPLC analysis. All samples were analyzed by RP-HPLC on a Vydac C18 analytical column. The column temperature was maintained at $50{ }^{\circ} \mathrm{C}$ and the flow rate was $1 \mathrm{~mL} / \mathrm{min}$. Two linear gradient elution methods were used. The first method was 110 min analyzing time with the following gradient: 0 min, $90 \%$ solvent $\mathrm{A} ; 15$ min, $75 \%$ solvent $\mathrm{A} ; 35$ min, $73 \%$ solvent $\mathrm{A} ; 50$ min, $72 \%$ solvent $\mathrm{A} ; 110$ min, $70 \%$ solvent $\mathrm{A}$. The second method was 40 min with gradient as 0 min, $90 \%$ solvent 
A; 40 min, 60\% solvent A. The absorbance was monitored at $229 \mathrm{~nm}$. The best concentration combinations of each aromatic thiol and its disulfide to fold reduced BPTI were determined. The initial folding rates and rate constants were determined by fitting the native protein yields versus the refolding time to a single exponential function protein yield $\%=\mathrm{A}\left(1-\mathrm{e}^{-k \mathrm{t}}\right)$. The folding results of reduce BPTI with redox buffers containing each aromatic thiol and disulfide were compared with the folding with aliphatic small molecules GSH and GSSG.

\subsection{Results and discussion}

\subsubsection{Folding of reduced BPTI with QAS thiol and disulfide}

\subsubsection{Folding of reduced BPTI analyzed with the 110 min method}

The redox buffers containing positively charged small molecule QAS thiol and its corresponding disulfide were used to fold reduced BPTI. When reduced BPTI was folded with different concentrations of QAS disulfide $(0.09,0.25$, and $0.5 \mathrm{mM})$, the folding reactions were quenched with $20 \mu \mathrm{L}$ of formic acid at 5, 15, 60, 240, 480, and $1440 \mathrm{~min}$ time points (Figure 19). All folding reaction samples were analyzed by RP-HPLC with the 110 min method. Folding of reduced BPTI in the absence of QAS thiol indicated slow folding rates and low native protein yield at $12 \mathrm{~h}$. About $75 \%$ of native BPTI was obtained with 0.25 and $0.5 \mathrm{mM}$ QAS disulfide in $12 \mathrm{~h}$.

When the folding of reduced BPTI was conducted with QAS disulfide $(0.09$ and 0.25 $\mathrm{mM})$ and different concentrations of QAS thiol $(1,2,5$ and $10 \mathrm{mM})$, about $90 \%$ of native protein was produced in $4 \mathrm{~h}$ at all folding conditions (Figure 20). The folding rate was dependent on the thiol concentration as the folding reaction was completed in a shorter time with higher concentrations of QAS thiol. More than $90 \%$ native protein was produced 
in $1 \mathrm{~h}$ under some folding conditions (Figure 21). Folding of reduced BPTI with $0.09 \mathrm{mM}$ QAS disulfide, and 5 and $10 \mathrm{mM}$ thiol showed that about $90 \%$ of native protein was produced in $1 \mathrm{~h}$, but the native protein percentage decreased with longer refolding time. Thiol oxidation and the increase of total protein peak area was observed under the folding conditions with higher QAS thiol concentrations. In order to minimize thiol oxidation, reaction samples at same folding conditions with QAS thiol and disulfide were quenched with different acids including phosphoric acid, $\mathrm{HCl}$ and formic acid. All acid quenched samples were analyzed by RP-HPLC. The results showed that quenching with $80 \mu \mathrm{L}$ of formic acid controlled thiol oxidation of the folding reactions effectively. Therefore, the following folding reaction samples were quenched with $80 \mu \mathrm{L}$ of formic acid prior to HPLC analysis. Two kinetically stable folding intermediates were observed at 38 and 42 min retention time during the folding of reduced BPTI with $0.25 \mathrm{mM}$ QAS disulfide and thiol, which were also correlated with the thiol concentration (Figure 22).

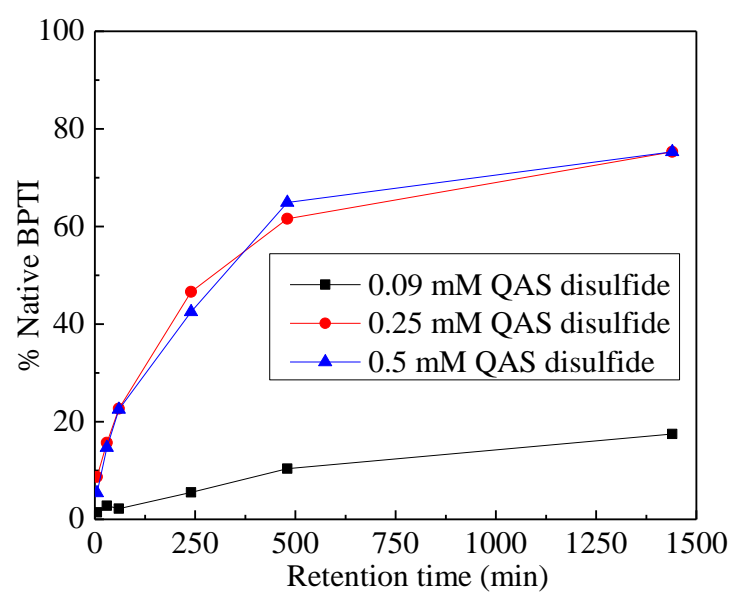

Figure 19 Folding of reduced BPTI with QAS disulfide only 


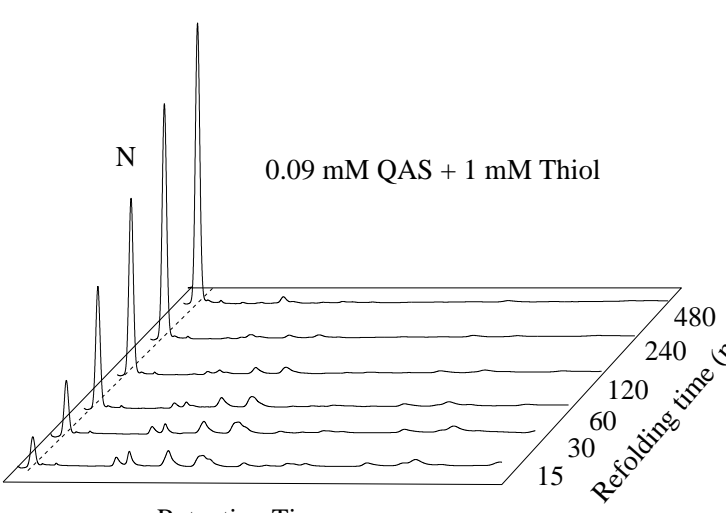

Retention Time

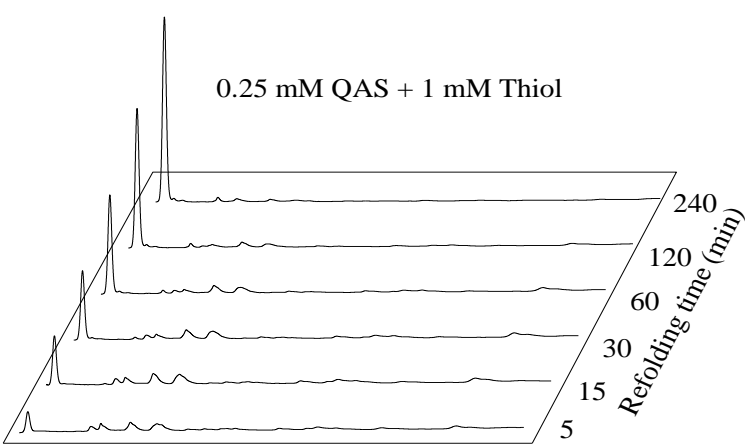

Retention time
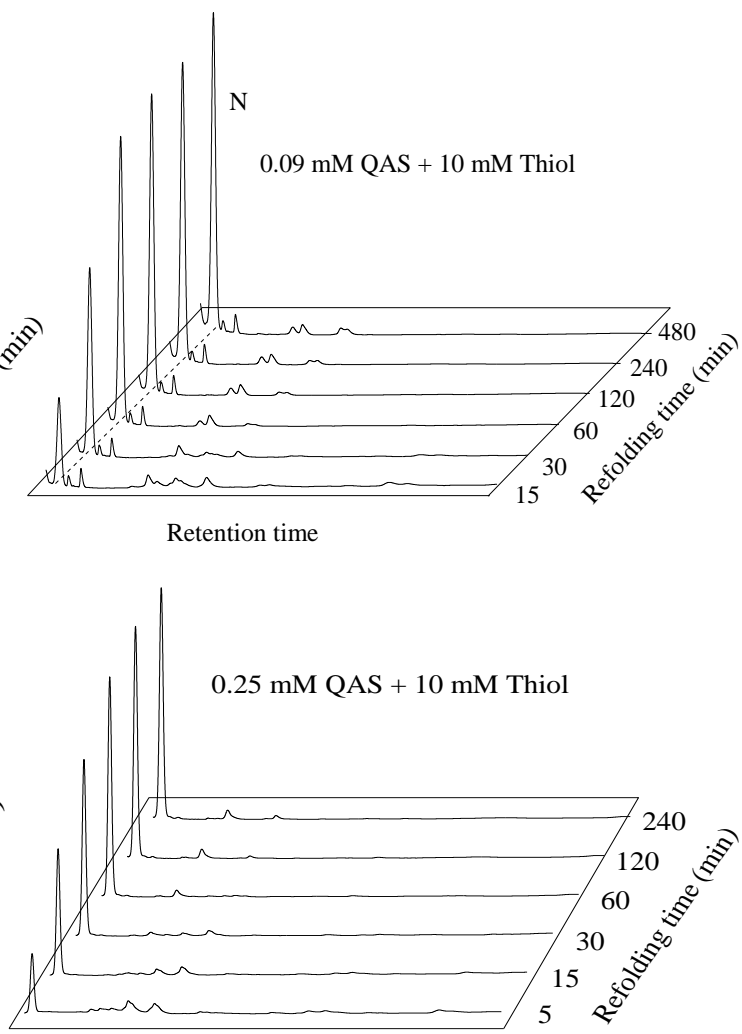

Retention time

Figure 20 HPLC chromatograms of reduced BPTI folding with QAS disulfide and thiol analyzed with the 110 min method
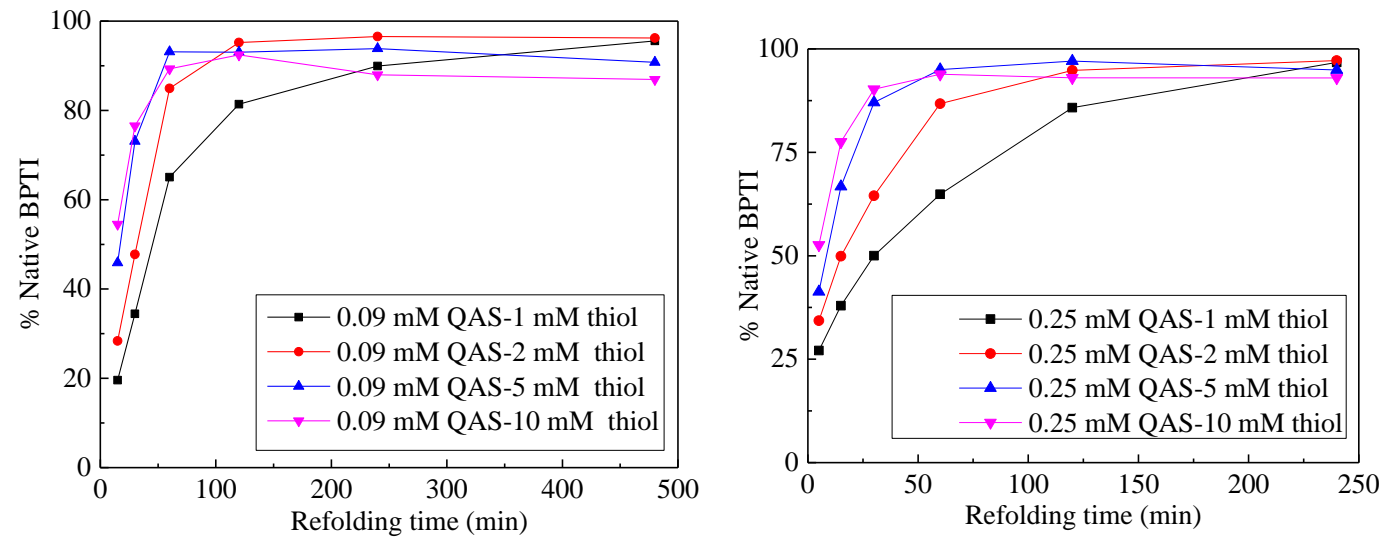

Figure 21 Folding of reduced BPTI with QAS disulfide and thiol analyzed with the 110 min method 

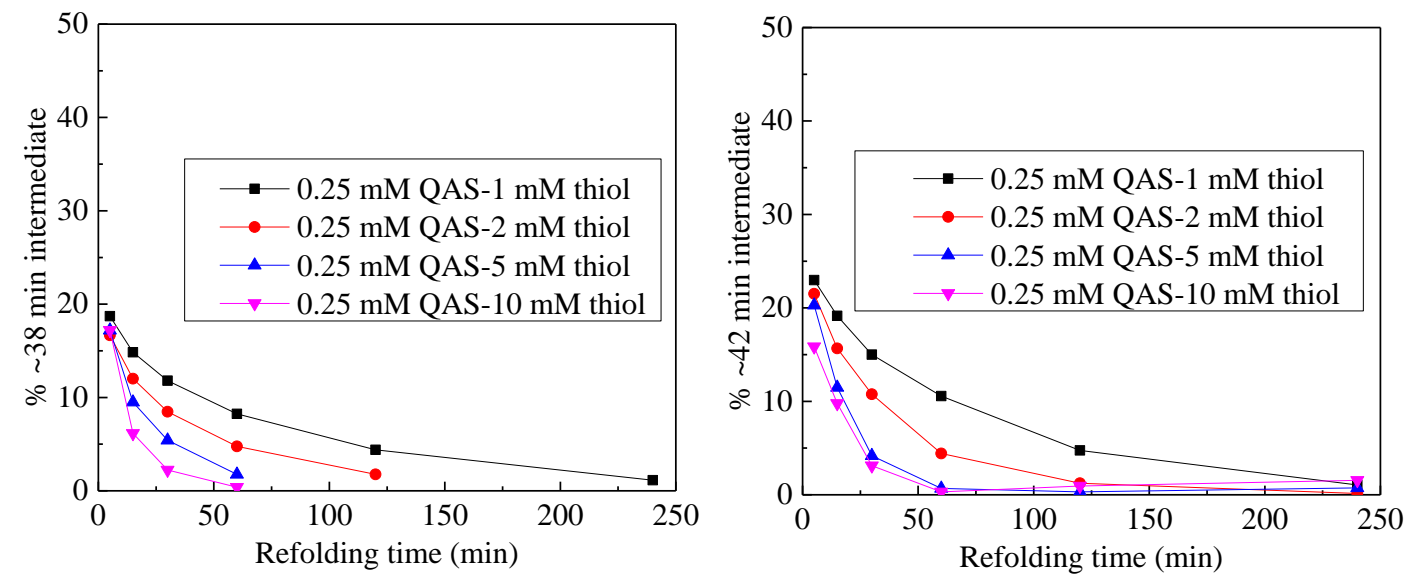

Figure 22 Folding intermediates of reduced BPTI with $0.25 \mathrm{mM}$ QAS disulfide and thiol

\subsubsection{Folding of reduced BPTI analyzed with the 40 min method}

To analyze folding reaction samples quickly, a new HPLC analysis method was developed by changing the elution gradient of the mobile phase. The following linear gradient was used: 0 min, $90 \%$ buffer A; $40 \mathrm{~min}, 60 \%$ buffer A, which only took $40 \mathrm{~min}$ to analyze each reaction sample. The validity of the new method was determined using the best condition of the traditional redox buffer containing $5 \mathrm{mM}$ GSSG and $5 \mathrm{mM} \mathrm{GSH}$, showing that lower native protein percentage was obtained than the results using the 110 min method in previous studies at the same time points. ${ }^{38}$ When reduced BPTI was folded with $5 \mathrm{mM}$ GSSG and $5 \mathrm{mM} \mathrm{GSH}$, almost $90 \%$ native protein was produced in $2 \mathrm{~d}$. More folding intermediates were observed from the HPLC chromatograms, leading to lower native protein yields (Figure 23).

Compared to the new method, the old method overestimated the percentage of native BPTI at different folding time points. The long analysis time broadened the folding intermediate peaks, resulting in the neglect of some folding intermediate peaks. Therefore, the new method was used to analyze the protein folding process faster. Additional folding 
intermediates were detected with the 40 min method analysis on HPLC chromatograms, which allowed the determination on more accurate native protein yields.

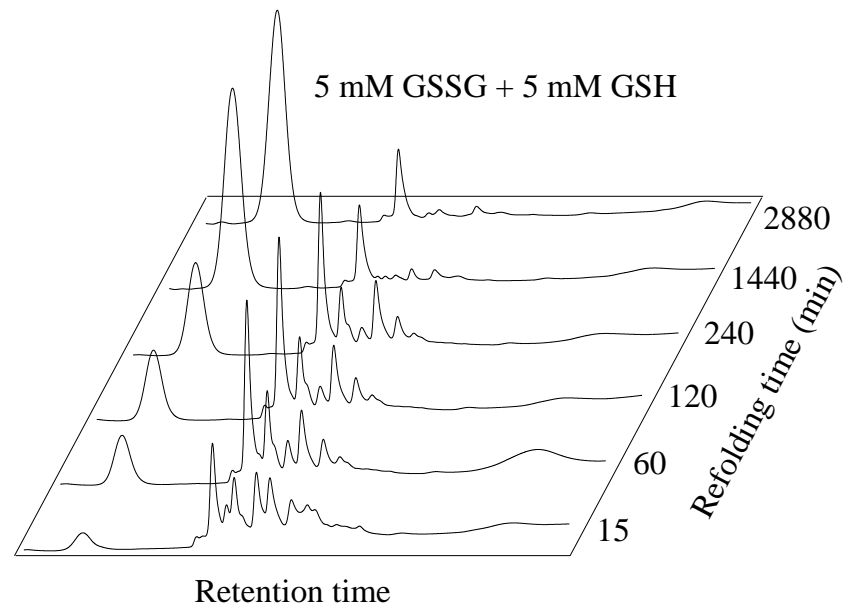

Figure 23 HPLC chromatograms of reduced BPTI folding with $5 \mathrm{mM}$ GSSG and $5 \mathrm{mM}$ GSH analyzed by the 40 min method

Folding reactions of reduced BPTI were conducted with different concentrations of QAS disulfide $(0.09,0.25,1$ and $2.5 \mathrm{mM})$ and QAS thiol $(1,2,5,10$ and $20 \mathrm{mM})$. All reaction samples were analyzed by RP-HPLC with the new 40 min method. The short method allowed the folding analysis to be completed in a shorter time, which also minimized the oxidation of folding reactions samples in air. Folding analysis using the new method confirmed that QAS disulfide and thiol folded reduced BPTI to its native form faster than GSSG and GSH.

Reduced BPTI was folded with 0.09 mM QAS and different concentrations of thiol (1, 2, 5 and $10 \mathrm{Mm}$ ) (Figure 24). With higher concentrations of QAS thiol, the folding rates of reduced protein were increased. When folding reduced BPTI with redox buffer consisted of $0.09 \mathrm{mM}$ disulfide and $10 \mathrm{mM}$ thiol, about $90 \%$ native protein was produced in $4 \mathrm{~h}$ (Figure 26). The folding rates were calculated by fitting the data of native protein percent yield against the refolding time to the single exponential function protein yield $\%=$ 
$\mathrm{A}\left(1-\mathrm{e}^{-k \mathrm{t}}\right)$. The initial folding rate of reduced BPTI with $0.09 \mathrm{mM}$ disulfide and $10 \mathrm{mM}$ thiol was $4.48 \pm 0.13 \% / \mathrm{min}$ and the maximal folding yield was calculated to be $90 \pm 1 \%$ (Table 1).

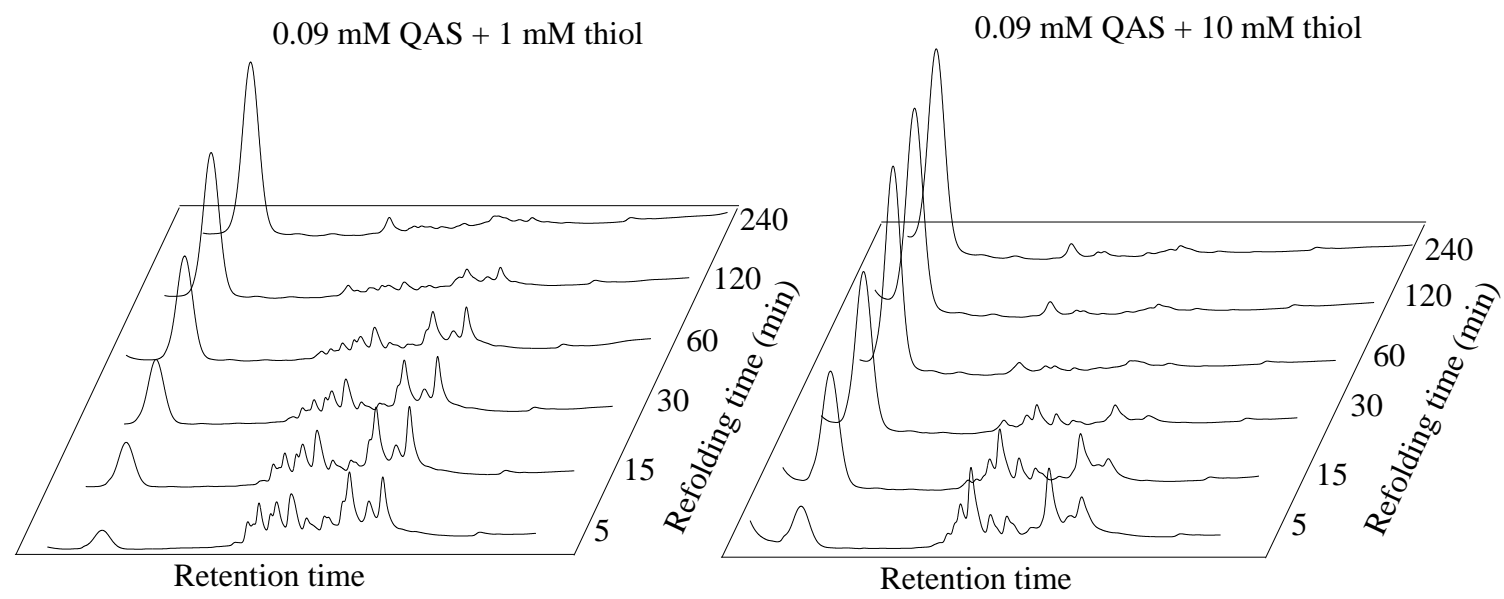

Figure 24 HPLC chromatograms of reduced BPTI folding with $0.09 \mathrm{mM}$ QAS disulfide and thiol analyzed with the 40 min method

When reduced BPTI was folded with $0.25 \mathrm{mM}$ QAS disulfide and different concentrations of QAS thiol $(1,2,5,10$ and $20 \mathrm{mM})$, about $90 \%$ of native BPTI was produced in $4 \mathrm{~h}$ under most of the folding conditions (Figure 25). With 0.25 mM QAS disulfide and $10 \mathrm{mM}$ thiol, the folding process was completed in $2 \mathrm{~h}$ in terms of the formation of $90 \%$ native protein (Figure 26). The maximal folding yield was calculated as $91 \pm 1 \%$ and the initial folding rate reached $4.90 \pm 0.23 \% / \mathrm{min}$ (Table 1 ). The redox buffer containing $0.25 \mathrm{mM}$ disulfide and $20 \mathrm{mM}$ QAS thiol was also used to fold reduced BPTI. The initial folding rate was increased, but the folding yield of native protein was slightly decreased. 


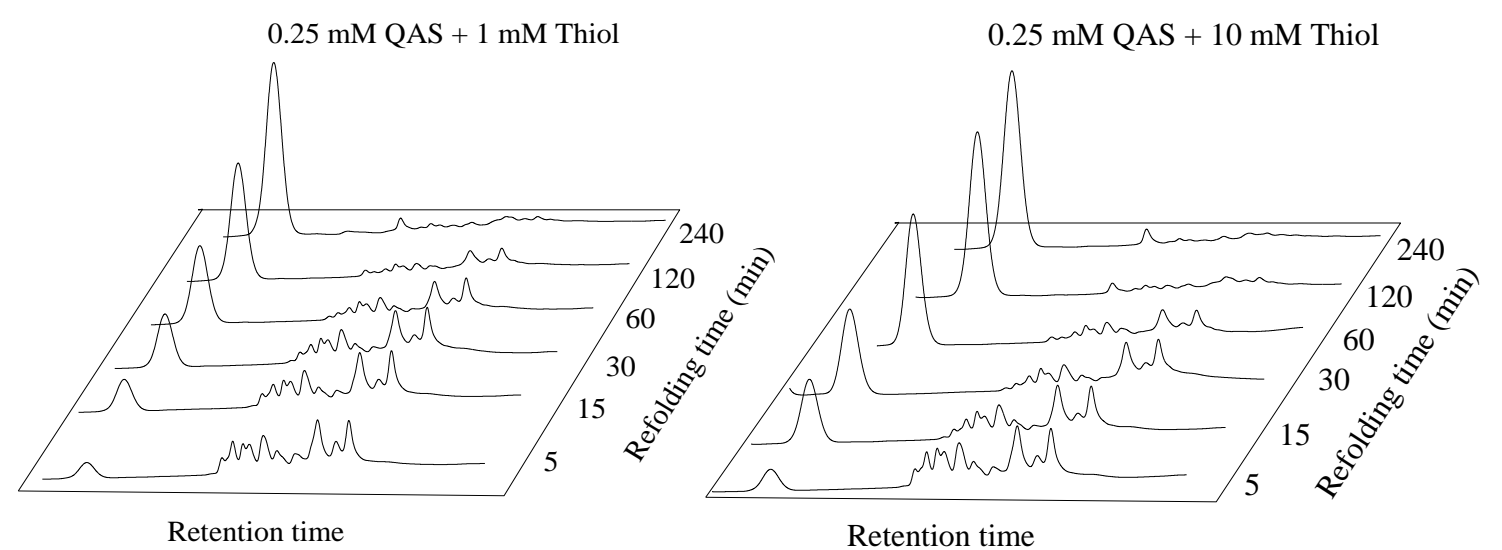

Figure 25 HPLC chromatograms of reduced BPTI folding with $0.25 \mathrm{mM}$ QAS disulfide and thiol analyzed with the 40 min method

When the folding of reduced BPTI was conducted with $1 \mathrm{mM}$ QAS disulfide and different concentrations of thiol $(1,2,5,10$ and $20 \mathrm{mM})$, the folding yields decreased because of the formation of mixed disulfide intermediates, compared to folding with 0.25 mM QAS disulfide and the same thiol concentrations. The initial folding rates also decreased when high concentration ratios of QAS disulfide to thiol were used in the redox buffer. The initial rates of folding with $1 \mathrm{mM}$ QAS disulfide, and 1 and $2 \mathrm{mM}$ thiol were determined to be 1.21 and $1.70 \% / \mathrm{min}$, respectively. With $1 \mathrm{mM}$ QAS disulfide and 10 $\mathrm{mM}$ thiol, the folding yield of native protein reached $88 \%$. According to the exponential curve model, the maximal folding yield was calculated to be $88 \pm 2 \%$, and the initial folding rate was $6.20 \pm 0.17 \% / \mathrm{min}$ (Table 1).

Low folding yields of native protein were produced when reduced BPTI was folded with 2.5 mM QAS disulfide and different concentrations of QAS thiol. The highest folding yield was $87 \%$ when folding with $2.5 \mathrm{mM}$ QAS disulfide and $10 \mathrm{mM}$ thiol. Folding of reduced BPTI with $2.5 \mathrm{mM}$ QAS disulfide, and 1 and $2 \mathrm{mM}$ thiol was not completed with high concentration ratios of QAS disulfide and thiol, as only about $67 \%$ and $73 \%$ of native 
protein were produced when the folding reactions were quenched at $4 \mathrm{~h}$ time point. High concentrations of QAS disulfide promoted the formation of mixed disulfide folding intermediates between protein and small molecule thiols. With slow rearrangement of mixed disulfide intermediates during the folding process, the folding of reduced BPTI with 2.5 mM QAS disulfide and QAS thiol became slow and low yielding (Table 1).
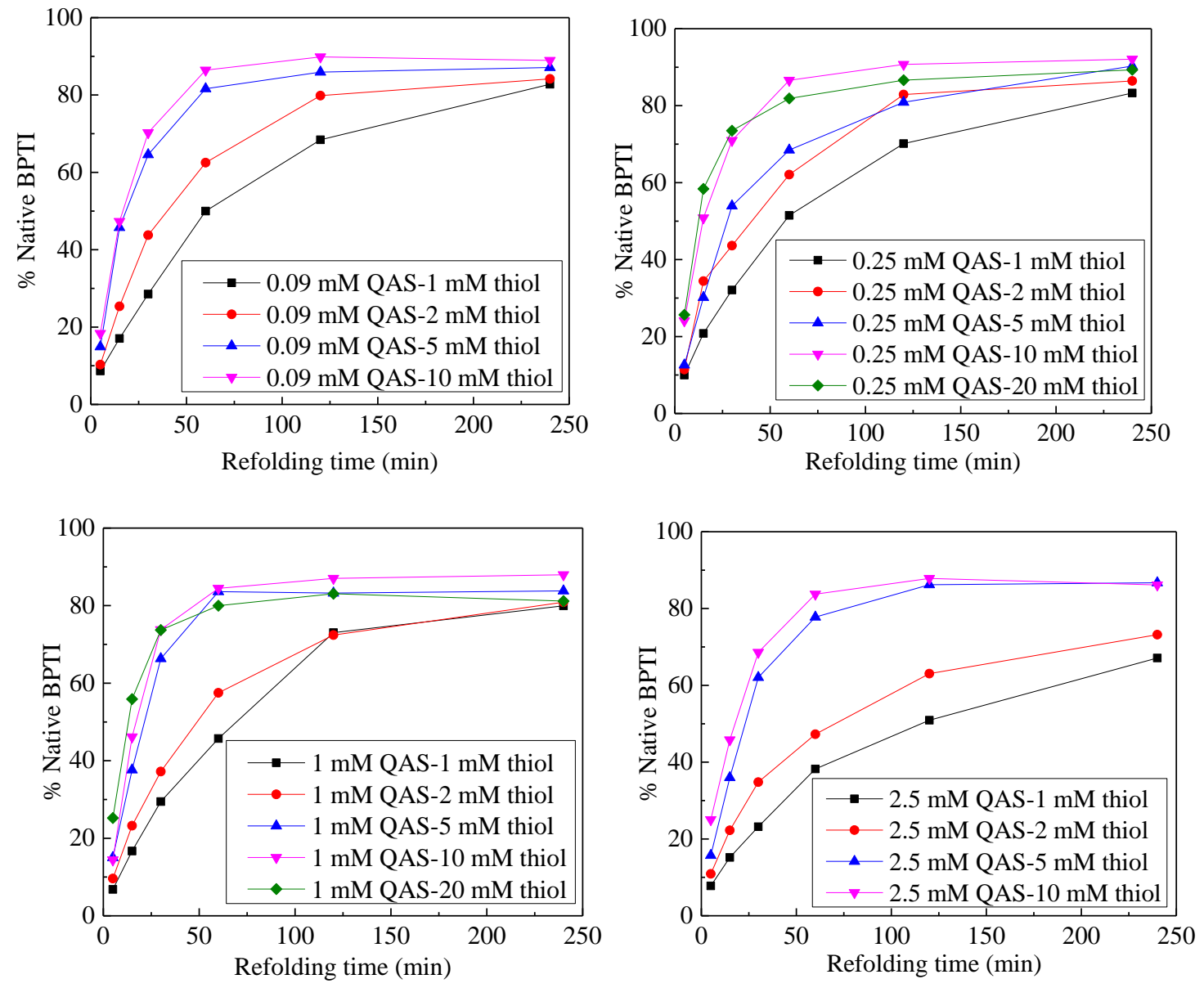

Figure 26 Folding of reduced BPTI with QAS disulfide and thiol analyzed with the $40 \mathrm{~min}$ method 
Table 1 Folding of reduced BPTI with QAS disulifide and thiol

\begin{tabular}{|c|c|c|c|c|}
\hline \multicolumn{2}{|c|}{ Redox buffers } & \multirow[b]{2}{*}{$\begin{array}{c}\mathrm{A} \\
\text { (\%native protein) }\end{array}$} & \multirow[b]{2}{*}{$\begin{array}{c}k \\
\left(\min ^{-1}\right)\end{array}$} & \multirow[b]{2}{*}{$\begin{array}{c}\text { Initial rate } \\
(\% / \mathrm{min})\end{array}$} \\
\hline $\begin{array}{l}\text { QAS disulfide } \\
(\mathrm{mM})\end{array}$ & $\begin{array}{c}\text { QAS thiol } \\
(\mathrm{mM})\end{array}$ & & & \\
\hline \multirow{4}{*}{0.09} & 1 & $85 \pm 2$ & $0.014 \pm 0.001$ & $1.22 \pm 0.08$ \\
\hline & 2 & $84 \pm 1$ & $0.024 \pm 0.001$ & $2.00 \pm 0.06$ \\
\hline & 5 & $87 \pm 1$ & $0.046 \pm 0.002$ & $4.03 \pm 0.22$ \\
\hline & 10 & $90 \pm 1$ & $0.050 \pm 0.001$ & $4.48 \pm 0.13$ \\
\hline \multirow{5}{*}{0.25} & 1 & $84 \pm 3$ & $0.016 \pm 0.001$ & $1.37 \pm 0.12$ \\
\hline & 2 & $86 \pm 4$ & $0.025 \pm 0.001$ & $2.17 \pm 0.30$ \\
\hline & 5 & $87 \pm 3$ & $0.029 \pm 0.002$ & $2.52 \pm 0.25$ \\
\hline & 10 & $91 \pm 1$ & $0.054 \pm 0.001$ & $4.90 \pm 0.23$ \\
\hline & 20 & $86 \pm 2$ & $0.071 \pm 0.001$ & $6.09 \pm 0.46$ \\
\hline \multirow{5}{*}{1} & 1 & $84 \pm 3$ & $0.014 \pm 0.001$ & $1.21 \pm 0.12$ \\
\hline & 2 & $80 \pm 1$ & $0.021 \pm 0.001$ & $1.70 \pm 0.08$ \\
\hline & 5 & $86 \pm 2$ & $0.045 \pm 0.004$ & $3.82 \pm 0.39$ \\
\hline & 10 & $88 \pm 2$ & $0.051 \pm 0.005$ & $4.51 \pm 0.45$ \\
\hline & 20 & $82 \pm 1$ & $0.076 \pm 0.002$ & $6.20 \pm 0.17$ \\
\hline \multirow{4}{*}{2.5} & 1 & $68 \pm 4$ & $0.014 \pm 0.002$ & $0.92 \pm 0.13$ \\
\hline & 2 & $71 \pm 3$ & $0.021 \pm 0.003$ & $1.49 \pm 0.20$ \\
\hline & 5 & $87 \pm 1$ & $0.039 \pm 0.002$ & $3.37 \pm 0.16$ \\
\hline & 10 & $87 \pm 2$ & $0.053 \pm 0.004$ & $4.64 \pm 0.34$ \\
\hline
\end{tabular}

\subsubsection{Folding of reduced BPTI with PA thiol and disulfide}

Folding of reduced BPTI was conducted in the presence of redox buffers containing negatively charged PA thiol and its corresponding disulfide. Since PA thiol has a higher pKa value of 6.6 than QAS thiol with pKa of 5.5, it is less reactive than QAS in thioldisulfide interchange reactions. Therefore, the time points to quench the reactions were set as $15,60,120,240,480$ and $1440 \mathrm{~min}$. The redox buffers containing of $0.09 \mathrm{mM}$ PA disulfide and 1, 2, 5 and $10 \mathrm{mM}$ PA thiol were used in the reduced BPTI folding reactions, and protein precipitation was observed at all folding conditions (Figure 27). Protein precipitation was proposed to be the result of the negative charge of PA thiol, which facilitated the formation of aggregated mixed disulfide intermediated. These intermediates 
formed between negatively charged PA thiol and positively charged BPTI protein during the early folding stage. ${ }^{84}$ The folding yields of native BPTI with different concentration combinations of PA thiol and disulfide were calculated on the basis of the protein peak area on the chromatograms, which represented the soluble form of the protein in the folding reactions. The results showed the folding process was completed in $24 \mathrm{~h}$ at all conditions in terms of the formation of about $90 \%$ of native BPTI when only analyzing the soluble forms (Figure 28).

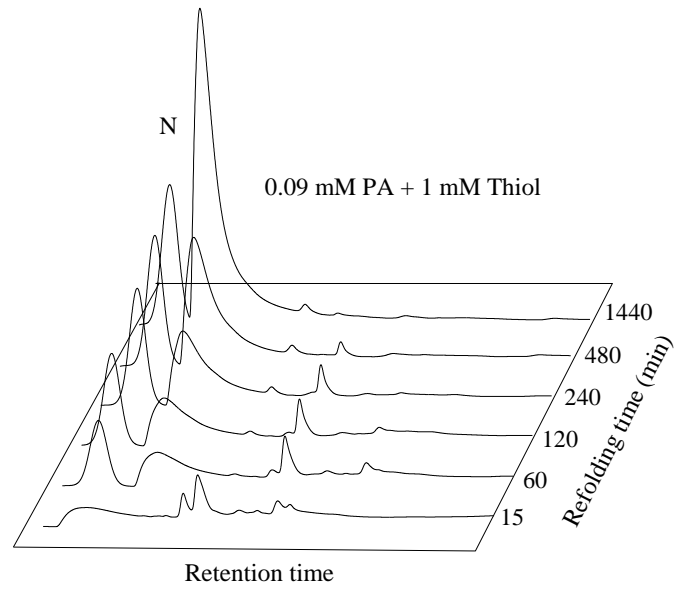

Figure 27 HPLC chromatograms of reduced BPTI with PA disulfide and thiol

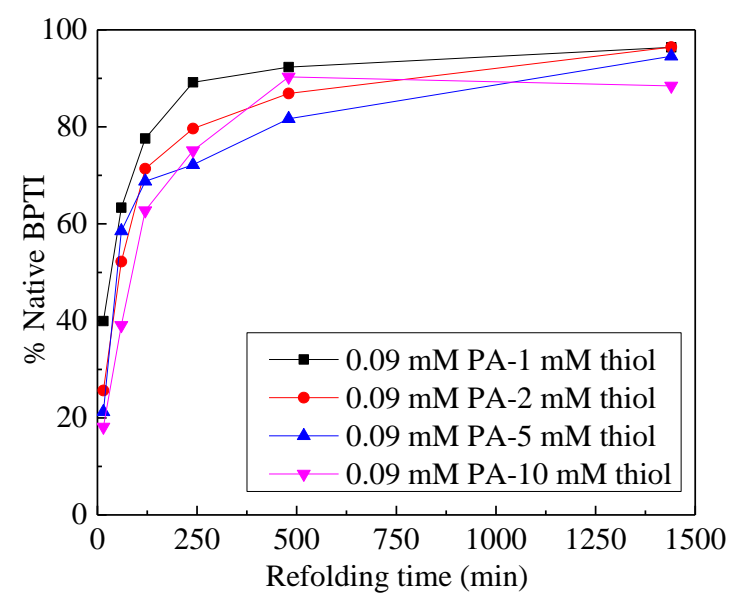

Figure 28 Folding of reduced BPTI with $0.09 \mathrm{mM}$ PA disulfide and thiol 
When the results of the folding reaction of reduced BPTI with PA thiol and disulfide were fitted to the exponential function, the folding rates and rate constants were calculated at different folding conditions. Native protein yields at different folding conditions with PA thiol and disulfide were affected by the protein precipitation issue, so the curve fits showed high relative errors. When reduced BPTI was folded with $0.09 \mathrm{mM}$ PA disulfide and $1 \mathrm{mM}$ thiol, native protein yield reached a maximum of $92 \% \pm 5 \%$ and the initial rate was determined to be $2.18 \pm 0.55 \% / \mathrm{min}$ (Table 2 ).

Table 2 Folding of reduced BPTI with PA disulifde and thiol

\begin{tabular}{|c|c|c|c|c|}
\hline \multicolumn{2}{|c|}{ Redox buffers } & \multirow[b]{2}{*}{$\underset{\text { (\%native protein) }}{\mathrm{A}}$} & \multirow[b]{2}{*}{$\begin{array}{c}k \\
\left(\min ^{-1}\right)\end{array}$} & \multirow[b]{2}{*}{$\begin{array}{l}\text { Initial rate } \\
(\% / \mathrm{min})\end{array}$} \\
\hline $\begin{array}{l}\text { PA disulfide } \\
(\mathrm{mM})\end{array}$ & $\begin{array}{l}\text { PA thiol } \\
(\mathrm{mM})\end{array}$ & & & \\
\hline \multirow{4}{*}{0.09} & $1 *$ & $92 \pm 5$ & $0.024 \pm 0.006$ & $2.18 \pm 0.55$ \\
\hline & $2^{*}$ & $88 \pm 4$ & $0.015 \pm 0.003$ & $1.33 \pm 0.25$ \\
\hline & $5^{*}$ & $83 \pm 5$ & $0.018 \pm 0.004$ & $1.50 \pm 0.38$ \\
\hline & $10^{*}$ & $88 \pm 3$ & $0.010 \pm 0.001$ & $0.88 \pm 0.10$ \\
\hline
\end{tabular}

*Protein precipitation occurred during folding.

\subsubsection{Folding of reduced BPTI with SA thiol and disulfide}

\subsubsection{Folding of reduced BPTI analyzed with the 110 min method}

Compared to PA thiol with two negative charges on the phosphonate group, SA thiol with only one negative charge was expected to minimize the occurrence of protein precipitation caused by charge effects of aromatic thiols and disulfides. Folding reactions of reduced BPTI were conducted with different concentrations of SA thiol and disulfide. Reduced BPTI was first folded with $0.25 \mathrm{mM}$ SA disulfide. Aliquots of $300 \mu \mathrm{L}$ were removed and quenched with $20 \mu \mathrm{L}$ formic acid at 15, 60, 120, 240, 480 and 720 min time points (Figure 29). Acid quenched reaction samples were analyzed by RP-HPLC with the 110 min method. The folding results showed that only $42 \%$ of native BPTI was produced 
in $12 \mathrm{~h}$ folding time. A kinetically stable folding intermediate was clearly observed at all folding time points, which was possibly a mixed disulfide intermediate that were difficult to convert to native protein because of the absence of reducing agents in thiol-disulfide rearrangement reactions.

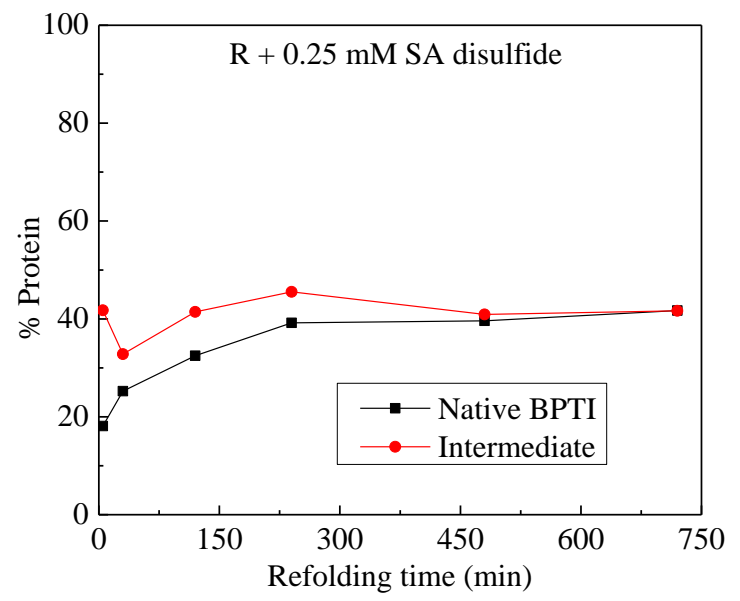

Figure 29 Folding of reduced BPTI with $0.25 \mathrm{mM}$ SA disulfide

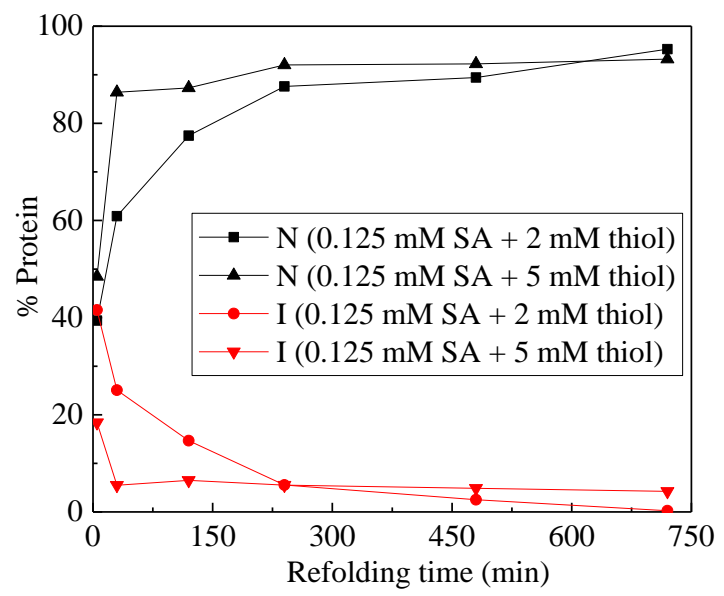

Figure 30 Folding of reduced BPTI with $0.125 \mathrm{mM}$ SA disulfide and thiol

The redox buffers containing both SA thiol and disulfide were used to fold reduced BPTI in order to have better thiol-disulfide interchange during folding. With $0.125 \mathrm{mM} \mathrm{SA}$ disulfide, and 2 and $5 \mathrm{mM}$ thiol, about $90 \%$ of native BPTI was produced in $480 \mathrm{~min}$ 
(Figure 30). The change in SA thiol concentration led to different folding rates and disappearance rates of folding intermediates. The folding rate of reduced BPTI with SA thiol and disulfide was thiol-dependent as the reaction with $5 \mathrm{mM}$ SA thiol showed a faster rate than that with $2 \mathrm{mM}$ SA thiol and the same SA disulfide concentration. When reduced BPTI was folded with $0.125 \mathrm{mM}$ and $5 \mathrm{mM}$ SA thiol, protein precipitation was observed during folding.

Then reduced BPTI was folded with a series of redox buffers containing different concentrations of SA disulfide $(0.09$ and $0.25 \mathrm{mM})$ and thiol $(1,2,5$ and $10 \mathrm{mM})$, respectively. At higher concentrations of SA, including $0.09 \mathrm{mM}$ SA disulfide, and 5 and $10 \mathrm{mM}$ thiol, and $0.25 \mathrm{mM}$ disulfide, protein precipitation was observed. The HPLC chromatograms showed low protein peak intensities due to protein loss by precipitation (Figure 31). The higher the concentration of small molecule thiol and disulfide, the greater the protein precipitation.

Nevertheless, native protein yield with SA thiol and disulfide was obtained using the soluble form of the protein in the reaction mixture. Protein peak areas were summed and the native protein percentage was calculated from HPLC chromatograms, reflecting that redox buffers containing SA thiol and disulfide facilitated the oxidative folding process of reduced BPTI. All folding reactions with $0.09 \mathrm{mM}$ SA disulfide and thiol were completed in $4 \mathrm{~h}$ in terms of the formation of $90 \%$ of native protein (Figure 32). Folding with 0.25 $\mathrm{mM}$ disulfide and all thiol concentration showed low native protein yield caused by large amounts of protein precipitates. 


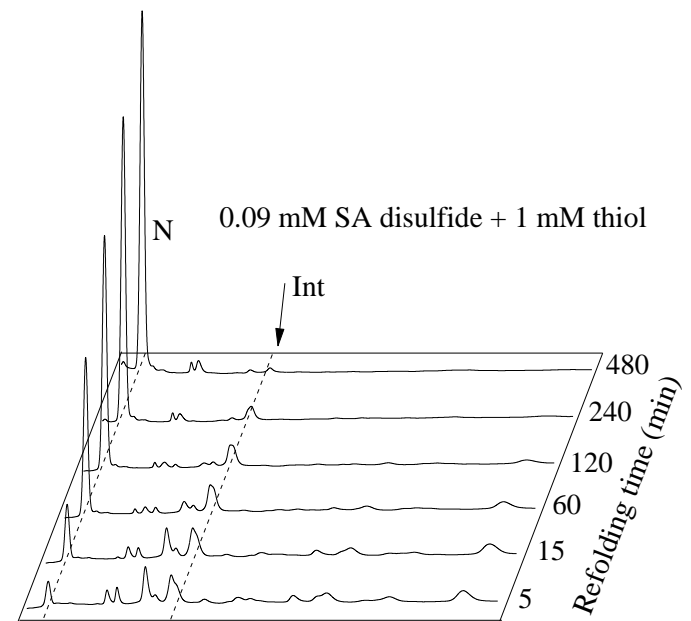

Retention time

$0.25 \mathrm{mM} \mathrm{SA}+1 \mathrm{mM}$ Thiol

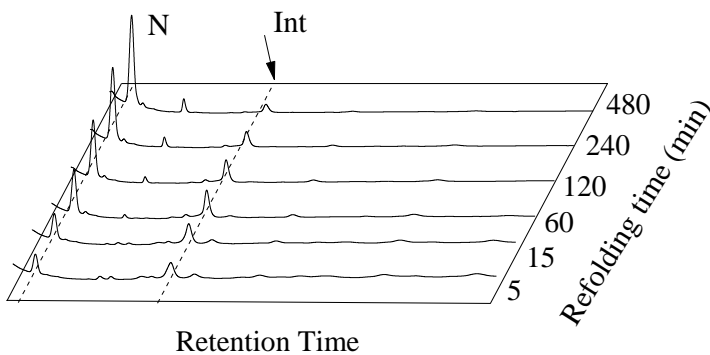

Retention Time

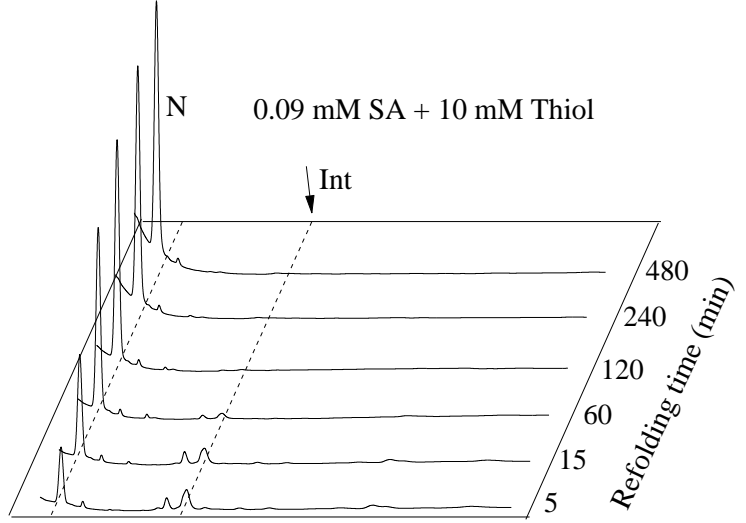

Retention Time

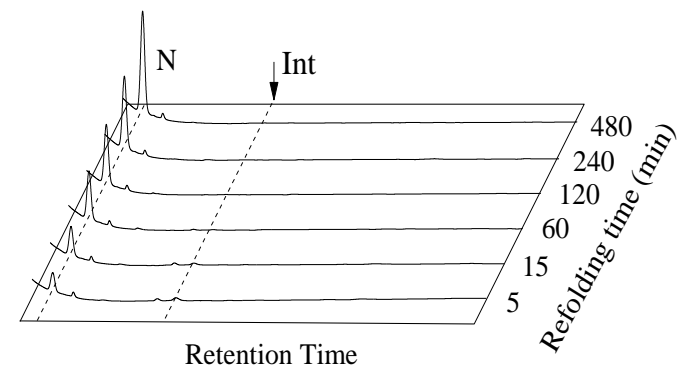

Figure 31 HPLC chromatograms of reduced BPTI with SA disulfide and thiol
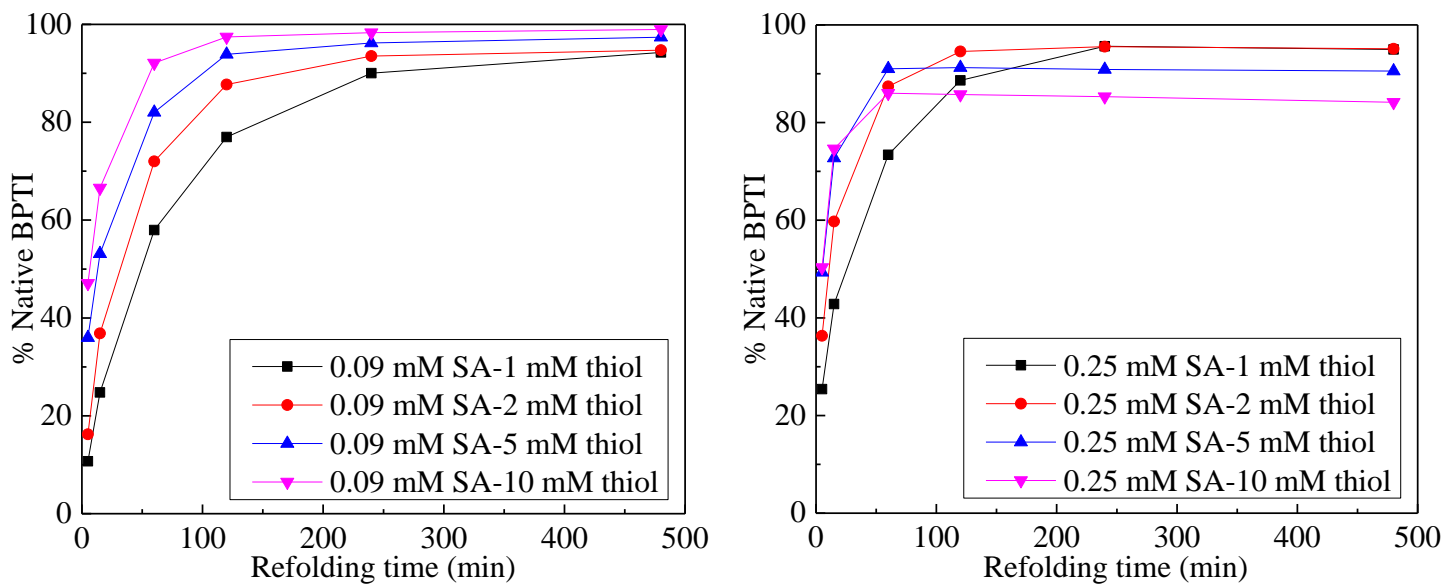

Figure 32 Folding of reduced BPTI with SA disulfide and thiol analyzed with the $110 \mathrm{~min}$ method 
One folding intermediate accumulated in all folding reactions with both 0.09 and 0.25 mM SA disulfide and various thiol concentrations according to the HPLC chromatograms. With $0.25 \mathrm{mM}$ SA disulfide and low thiol concentrations, additional folding intermediates accumulated owing to low thiol-disulfide interchange activity. Increasing the SA thiol concentration with $0.25 \mathrm{mM}$ SA disulfide helped the mixed disulfides rearrange, thus the folding intermediate concentrations decreased (Figure 33).
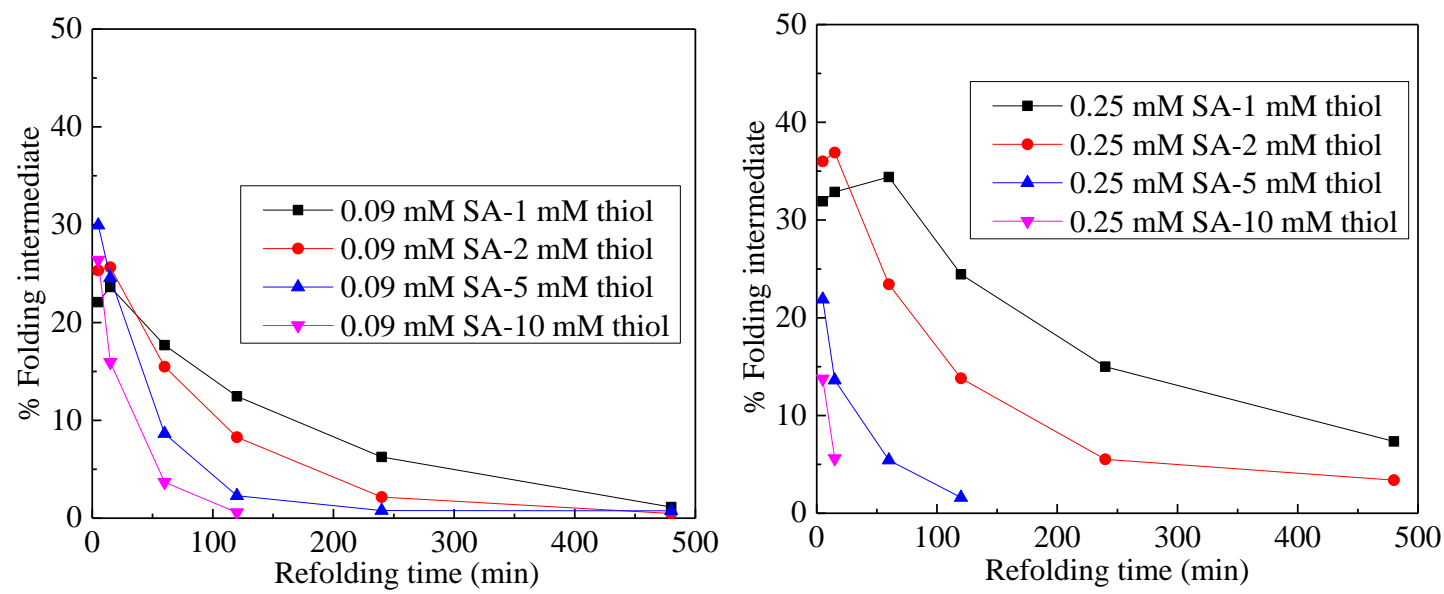

Figure 33 Folding intermediates of reduced BPTI folding with SA disulfide and thiol

Table 3 Folding of reduced BPTI with SA disulifde and thiol analyzed with the 110 min method

\begin{tabular}{|c|c|c|c|c|}
\hline \multicolumn{2}{|c|}{ Redox buffers } & \multirow{2}{*}{ (\%native protein) } & \multirow{2}{*}{$\begin{array}{c}k \\
\left(\min ^{-1}\right)\end{array}$} & \multirow{2}{*}{$\begin{array}{l}\text { Initial rate } \\
(\% / \mathrm{min})\end{array}$} \\
\hline $\begin{array}{l}\text { SA disulfide } \\
(\mathrm{mM})\end{array}$ & $\begin{array}{l}\text { SA thiol } \\
(\mathrm{mM})\end{array}$ & & & \\
\hline \multirow{4}{*}{0.09} & 1 & $92 \pm 2$ & $0.017 \pm 0.002$ & $1.56 \pm 0.15$ \\
\hline & 2 & $92 \pm 2$ & $0.029 \pm 0.003$ & $2.72 \pm 0.31$ \\
\hline & $5^{*}$ & $93 \pm 4$ & $0.065 \pm 0.013$ & $6.02 \pm 1.26$ \\
\hline & $10 *$ & $96 \pm 3$ & $0.100 \pm 0.017$ & $9.65 \pm 1.67$ \\
\hline \multirow{2}{*}{0.125} & 2 & $89 \pm 5$ & $0.024 \pm 0.006$ & $2.11 \pm 0.53$ \\
\hline & $5 *$ & $91 \pm 1$ & $0.050 \pm 0.003$ & $4.57 \pm 0.32$ \\
\hline \multirow{4}{*}{0.25} & $1 *$ & $91 \pm 4$ & $0.039 \pm 0.008$ & $3.59 \pm 0.78$ \\
\hline & $2 *$ & $93 \pm 2$ & $0.077 \pm 0.010$ & $7.18 \pm 0.92$ \\
\hline & $5^{*}$ & $90 \pm 2$ & $0.137 \pm 0.015$ & $12.39 \pm 1.36$ \\
\hline & $10 *$ & $85 \pm 1$ & $0.169 \pm 0.011$ & $14.35 \pm 0.99$ \\
\hline
\end{tabular}

*Protein precipitation occurred during folding. 
The folding rates of reduced BPTI folding with SA thiol and disulfide were determined by fitting the native protein yield versus refolding time to a single exponential function (Table 3). The function is defined asprotein yield $\%=\mathrm{A}\left(1-\mathrm{e}^{-k \mathrm{t}}\right)$, where $\mathrm{A}$ is the maximum protein yield and $k$ is the folding rate constant. The initial folding rates were calculated as $\mathrm{A} * k$ at different concentrations of SA thiol and disulfide. The best folding concentration was defined as the redox buffer concentration combinations of SA thiol and disulfide at which the initial folding rate reached a maximum. Since protein precipitation was observed during folding at high concentrations of SA thiol and disulfide, the best folding condition without protein precipitation was found to be $0.09 \mathrm{mM}$ SA disulfide and $2 \mathrm{mM}$ thiol, which showed the folding rate constant $k=0.029 \pm 0.003 \mathrm{~min}^{-1}$, and the initial folding rate $2.72 \pm 0.31 \% / \mathrm{min}$.

\subsubsection{Folding of reduced BPTI analyzed with the 40 min method}

Faster folding analysis of reduced BPTI with SA disulfide and thiol was achieved with RP-HPLC using the 40 min method. Compared to the analysis with the same folding conditions using the 110 min method, lower folding yields and rates were obtained when the reactions were analyzed using the 40 min method. Less than $90 \%$ of native protein was produced in $8 \mathrm{~h}$ folding time. Protein precipitation was also observed at higher concentrations of SA disulfide and thiol. With $0.09 \mathrm{mM} \mathrm{SA}$ disulfide and $2 \mathrm{mM}$ thiol, about $85 \%$ of native protein was obtained in $8 \mathrm{~h}$, and the initial folding rate was calculated to be $0.89 \pm 0.28 \% / \mathrm{min}$ 

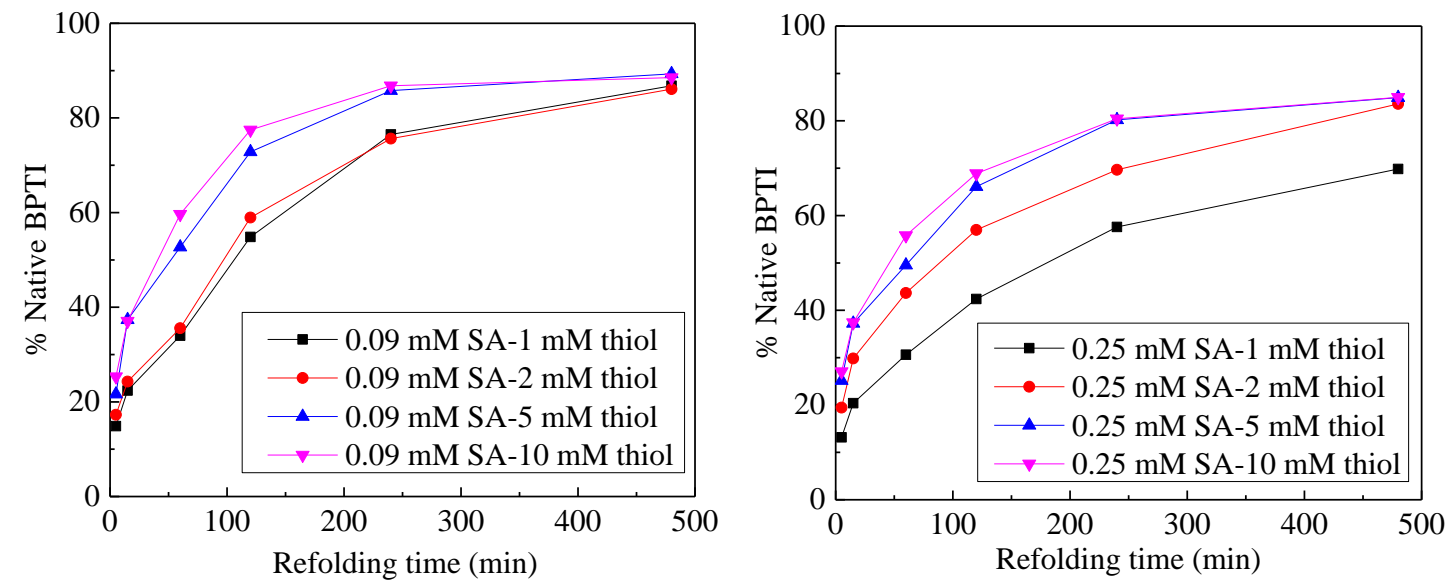

Figure 34 Folding of reduced BPTI with SA disulfide and thiol analyzed with the 40 min method

Table 4 Folding of reduced BPTI with SA disulifde and thiol analyzed with the 40 min method

\begin{tabular}{|c|c|c|c|c|}
\hline \multicolumn{2}{|c|}{ Redox buffers } & \multirow[b]{2}{*}{$\begin{array}{c}\mathrm{A} \\
(\% \text { native protein) }\end{array}$} & \multirow[b]{2}{*}{$\begin{array}{c}k \\
\left(\min ^{-1}\right)\end{array}$} & \multirow[b]{2}{*}{$\begin{array}{c}\text { Initial rate } \\
(\% / \mathrm{min})\end{array}$} \\
\hline $\begin{array}{l}\text { SA disulfide } \\
(\mathrm{mM})\end{array}$ & $\begin{array}{l}\text { SA thiol } \\
(\mathrm{mM})\end{array}$ & & & \\
\hline \multirow{4}{*}{0.09} & 1 & $87 \pm 8$ & $0.009 \pm 0.002$ & $0.79 \pm 0.22$ \\
\hline & 2 & $84 \pm 8$ & $0.011 \pm 0.003$ & $0.89 \pm 0.28$ \\
\hline & $5 *$ & $84 \pm 7$ & $0.023 \pm 0.008$ & $1.93 \pm 0.70$ \\
\hline & $10^{*}$ & $83 \pm 6$ & $0.032 \pm 0.010$ & $2.63 \pm 0.85$ \\
\hline \multirow{4}{*}{0.25} & $1 *$ & $67 \pm 8$ & $0.010 \pm 0.003$ & $0.67 \pm 0.23$ \\
\hline & $2 *$ & $75 \pm 9$ & $0.016 \pm 0.007$ & $1.23 \pm 0.51$ \\
\hline & $5 *$ & $77 \pm 8$ & $0.028 \pm 0.013$ & $2.15 \pm 0.99$ \\
\hline & $10 *$ & $75 \pm 6$ & $0.042 \pm 0.017$ & $3.17 \pm 1.28$ \\
\hline
\end{tabular}

*Protein precipitation occurred during folding.

\subsection{Conclusion}

Oxidative folding of reduced BPTI was studied in the presence of positively or negatively charged $p$-substituted aromatic thiols and their corresponding disulfides. Compared to aliphatic thiols, such as GSH, all three aromatic thiols, SA, PA and QAS, were shown to facilitate the folding of reduced BPTI. Previous studies showed that about $90 \%$ of native BPTI was produced in $48 \mathrm{~h}$ with $5 \mathrm{mM}$ GSSG and $5 \mathrm{mM}$ GSH, which has 
been determined to be the best concentration combinations of GSSG and GSH. In comparison, about $90 \%$ of native protein was obtained within $8 \mathrm{~h}$ at all folding conditions of redox buffers containing SA thiol and disulfide. Folding of reduced BPTI with PA thiol and disulfide produced about $90 \%$ native protein in $24 \mathrm{~h}$. With all folding concentration combinations of QAS disulfide and thiol, the best condition to fold reduced BPTI was determined to be $0.25 \mathrm{mM}$ QAS disulfide and $10 \mathrm{mM}$ thiol in terms of the time to produce $90 \%$ of native protein. With $0.25 \mathrm{mM}$ QAS disulfide and $10 \mathrm{mM}$ thiol, about $90 \%$ of the native protein was produced in $2 \mathrm{~h}$, while after $2 \mathrm{~h}$, only $25 \%$ of native BPTI was obtained with $5 \mathrm{mM}$ GSH and $5 \mathrm{mM}$ GSSG (Figure 35). The maximal folding yields and initial folding rates were calculated on the basis of the relationship between the native protein yield and the refolding time. When reduced BPTI was folded with $5 \mathrm{mM} \mathrm{GSSG}$ and $5 \mathrm{mM}$ GSH, the initial folding rate was $0.21 \pm 0.04 \% / \mathrm{min}$. In comparison, folding with three aromatic thiols and their corresponding disulfides, including QAS, PA and SA, increased the initial folding rates by up to 20 times (Figure 36).

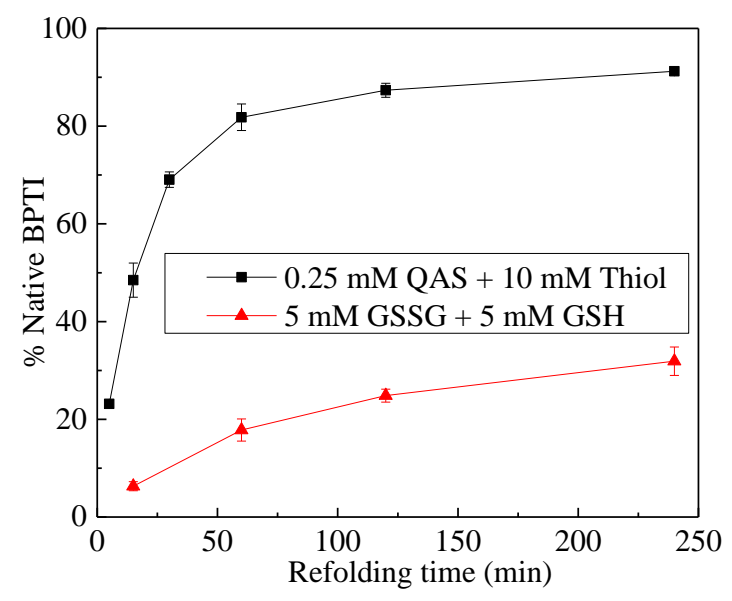

Figure 35 Comparison of reduced BPTI folding with GSSG/GSH and QAS disulfide/thiol 


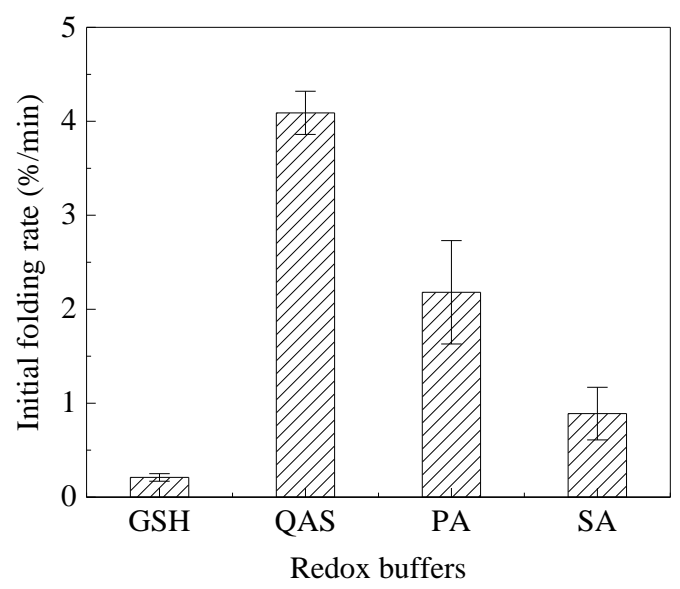

Figure 36 The charge effect of small molecules on the folding of reduced BPTI *Protein precipitation occurred during the folding with PA thiol and its disulfide

Protein precipitation was observed during the folding with PA and SA thiols and their corresponding disulfides. Since the folding reactions were all conducted at $\mathrm{pH} 7.3$, reduced BPTI with an isoelectric point (pI) of 10.5 was positively charged during folding, and aromatic thiols SA and PA were both negatively charged. During the protein folding process, mixed disulfide intermediates that formed disulfide bonds between protein and SA or PA thiol were produced through thiol-disulfide interchange reactions. Therefore, the net charge of mixed disulfide intermediates could become close to zero, leading to the occurrence of protein precipitation. However, folding of reduced BPTI with positively charged QAS thiol and disulfide showed no protein precipitation at all folding conditions. 


\section{Investigation of hydrophobicity effects of aromatic thiols on reduced BPTI folding}

\subsection{Abstract}

Aromatic thiols and disulfides with varying hydrophobicity were shown to have different efficiencies on the folding of disulfide-containing proteins. Four aromatic thiols with different lengths of an alkyl group on the aromatic ring, called methyl (QAS), butyl, hexyl and octyl thiols, were synthesized in the lab. All four thiols and their corresponding disulfides were used as redox buffers to fold reduced BPTI in vitro in the present study. Compared to the folding with QAS thiol and disulfide, folding of reduced BPTI with hexyl thiol and disulfide showed faster folding rates and yields. About $90 \%$ of native protein was produced in $1 \mathrm{~h}$ with the best concentrations of hexyl thiol and its disulfide. Protein precipitation was observed during the folding with high concentrations of hexyl and octyl thiols and their corresponding disulfides, indicating that the longer the hydrocarbon chain of the small molecule aromatic thiols, the more likely protein precipitation will occur. About $85 \%$ of native protein was produced in $4 \mathrm{~h}$ when folding with butyl thiol and its disulfide due to the formation of a prominent kinetically stable folding intermediate.

\subsection{Introduction}

Oxidative folding of disulfide-containing proteins involves thiol-disulfide interchange reactions. Folding of reduced BPTI under traditional redox buffer conditions showed the accumulation of many kinetically stable folding intermediates, such as $\mathrm{N}^{\prime}$ and $\mathrm{N}^{*}$. The remaining free thiols in $\mathrm{N}^{\prime}$ and $\mathrm{N}^{*}$ were buried in the hydrophobic core of the structure and were not accessible to solvents. The conversion of these folding intermediates to native proteins are usually the rate-limiting steps of the folding process. In order to improve the folding rates of disulfide-containing proteins, aromatic thiols and disulfides were used as 
redox buffers to fold proteins in vitro. As shown in a previous chapter, folding of reduced BPTI with aromatic thiols and their corresponding disulfides achieved faster folding rates and yields compared to folding with GSH and GSSG. Some kinetically stable folding intermediates were observed and remained throughout the folding process, which prolonged the folding process. Newly synthesized aromatic thiols with an elongated alkyl group on the aromatic ring, including butyl, hexyl, and octyl thiols, were expected to increase interactions with the hydrophobic core of disulfide-containing proteins s during folding, allowing more facile access to buried disulfide bonds (Figure 37). ${ }^{85}$ Therefore, the buried thiols in the hydrophobic core of protein folding intermediates can be more easily accessed by aromatic small molecules, and the intermediates can then be oxidized and rearranged to the native form of the protein more efficiently.

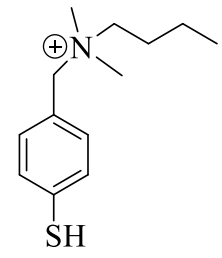

7

Butyl thiol

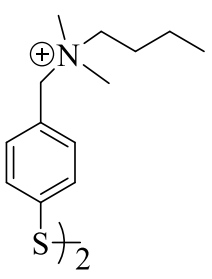

10

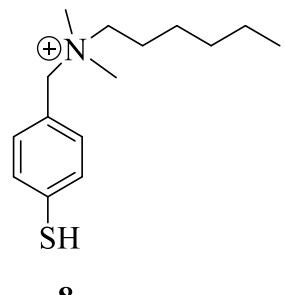

8

Hexyl thiol

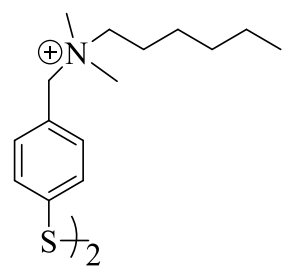

11

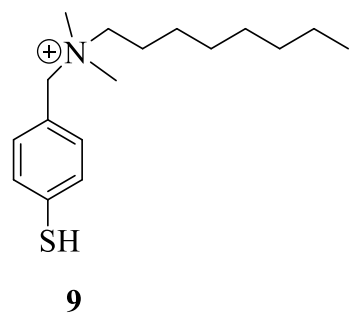

Octyl thiol

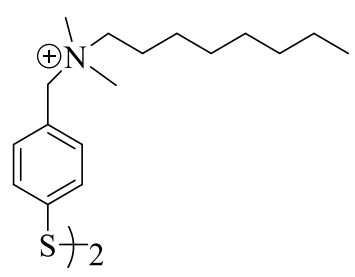

12

Octyl disulfide

Figure 37 Structures of aromatic butyl, hexyl, and octyl thiols and disulfides 


\subsection{Experimental section}

\subsubsection{Preparation of butyl, hexyl, and octyl thiols and disulfides}

Aromatic thiols with different elongated alkyl groups, including butyl, hexyl, and octyl thiols, were synthesized in the lab. Aromatic disulfides were prepared by oxidizing their corresponding thiols directly by atmosphere oxygen. Aromatic thiol solutions were stirred in air until the thiol was oxidized to its corresponding disulfide completely. The three aromatic thiols and disulfides were purified by RP-HPLC on a Vydac C18 semi-preparative column. For each injection, $2 \mathrm{~mL}$ of the sample solution was loaded on to the HPLC manually. Two solvents were used in the mobile phase. Solvent A was $0.1 \%$ TFA in water, and solvent B was $90 \%$ acetonitrile in water with $0.1 \%$ TFA. A linear gradient elution was utilized: 0 min, 90\% solvent A; 4 min, 85\%, 20 min, 75\% solvent A; 30 min, $60 \%$ solvent A; $50 \mathrm{~min}, 55 \%$ solvent $\mathrm{A}, 70 \mathrm{~min}, 50 \%$ solvent $\mathrm{A}$. A flow rate of $3 \mathrm{~mL} / \mathrm{min}$ was used and the HPLC column temperature was maintained at $50{ }^{\circ} \mathrm{C}$. The absorbance was monitored at $252 \mathrm{~nm}$. The purity of collected sample fractions was then analyzed by RP-HPLC on a Vydac C18 analytical column. The retentions time of the three aromatic thiols and disulfides were also determined with a gradient of 0 min, $90 \%$ solvent A; 40 min, 60\% solvent A. All pure fractions containing aromatic thiols or disulfides were combined and lyophilized. The lyophilized small molecule samples were dissolved in deoxygenated water and stored at $-20{ }^{\circ} \mathrm{C}$ for future use.

\subsection{2 pKa determination of three aromatic thiols using UV-vis method}

The pKa values of the three aromatic thiols were determined using a UV-Vis method on a spectrophotometer. ${ }^{83,86}$ Buffer solutions with different $\mathrm{pH}$ values were prepared with a concentration of $50 \mathrm{mM}$. The buffers were chosen as follows: glycine-HCl buffer, $\mathrm{pH} 2.5$, 
3.0, 3.3; 2,2-dimethylsuccinate buffer, $\mathrm{pH} 3.7,4.0,4.3,4.7,5.0,5.3,5.7,6.0,6.3,6.7$; Tris$\mathrm{HCl}$ buffer, $\mathrm{pH}$ 7.0, 7.3, 7.7, 8.0, 8.3, 8.7; glycine- $\mathrm{NaOH}$ buffer, $\mathrm{pH}$ 9.0, 9.5, 10.0, 10.5; $\mathrm{H}_{3} \mathrm{PO}_{4}$ buffer, $\mathrm{pH}$ 11. Each lyophilized thiol was dissolved in deoxygenated water to have a final concentration of $1.5 \mathrm{mM}$. Then $100 \mu \mathrm{L}$ of the thiol solution was added to $900 \mu \mathrm{L}$ of each buffer solution with varying $\mathrm{pH}$. The UV spectrum of each diluted solution was measured on a UV-Vis spectrophotometer. All three thiols and their corresponding thiolates had distinguishable spectra. The $\lambda_{\max }$ values of each thiol and the corresponding thiolate were determined from UV spectra. The absorbance data at the $\lambda_{\max }$ of the thiol were plotted as a function of buffer $\mathrm{pH}$. The plot was compared with plot derived from theory. The pKa value of each aromatic thiol was calculated from the best-fit curve.

\subsubsection{Folding of reduced BPTI with aromatic thiols and disulfides}

Reduced BPTI and $1.5 \times$ refolding buffer $(\mathrm{pH} 7.3)$ were prepared as described previously. Reduced BPTI was diluted to a final concentration of $30 \mu \mathrm{M}$ with $1.5 \times$ refolding buffer $(\mathrm{pH}$ 7.3). Reduced BPTI was folded in the presence of different concentration combinations of aromatic thiols $(1,2,5$ and $10 \mathrm{mM})$ and their corresponding disulfides $(0.09,0.125,0.25,1$ and $2.5 \mathrm{mM})$. Folding reactions of reduced BPTI were conducted in a $25{ }^{\circ} \mathrm{C}$ water bath under argon at $\mathrm{pH}$ 7.3. At certain folding time points, 300 $\mu \mathrm{L}$ aliquots of the reaction mixture were removed and quenched with $80 \mu \mathrm{L}$ of formic acid and stored on an ice bath immediately. All three aromatic disulfides had retention times longer than that of native protein when the quenched folding reaction samples were analyzed by RP-HPLC directly, and the peak corresponding to aromatic disulfides overlapped with the protein folding intermediates on HPLC chromatograms. Therefore, an additional gel filtration step was utilized to remove small molecule thiols and disulfides 
from the acid-quenched reaction mixtures prior to HPLC analysis. Acid-quenched folding samples were purified on a pipet column with Sephadex G-25 as the solid phase and 0.01 $\mathrm{N} \mathrm{HCl}$ as the mobile phase. Sample fractions containing proteins after gel filtration were collected and then analyzed on a Vydac C18 analytical column. A linear gradient elution was utilized: 0 min, $90 \%$ solvent $\mathrm{A} ; 40 \mathrm{~min}, 60 \%$ solvent $\mathrm{A}$. The flow rate was $1 \mathrm{~mL} / \mathrm{min}$. The absorbance was monitored at $229 \mathrm{~nm}$ and the temperature of the analytical column was maintained at $50{ }^{\circ} \mathrm{C}$. The best concentration combinations of each aromatic thiol and its corresponding disulfide in terms the refolding time to obtain $90 \%$ native protein were determined. The rate constants and initial folding rates were calculated by fitting the native protein yield $(\%)$ versus the refolding time (min) to a single exponential function, protein yield $\%=\mathrm{A}\left(1-\mathrm{e}^{-k \mathrm{t}}\right)$, where $\mathrm{A}$ is the maximal folding yield and $k$ is the folding rate constant.

\subsection{Results and discussion}

\subsection{1 pKa determination of aromatic thiols}

Aromatic thiols were diluted with 24 buffers with various $\mathrm{pH}$. The UV spectra of all diluted solutions measured with a UV-Vis spectrophotometer. All three aromatic thiols and their corresponding thiolates obtained distinguishable spectra (Figure 38). The thiol absorbed at $252 \mathrm{~nm}$ and the corresponding thiolate absorbed at $282 \mathrm{~nm}$. The isosbestic point of all three aromatic thiols was at $262 \mathrm{~nm}$. The plot was made using the absorbance at $252 \mathrm{~nm}$ against buffer $\mathrm{pH}$, and compared with the theoretical plot. All thiol pKa values were found to be 5.5 (Figure 39). 


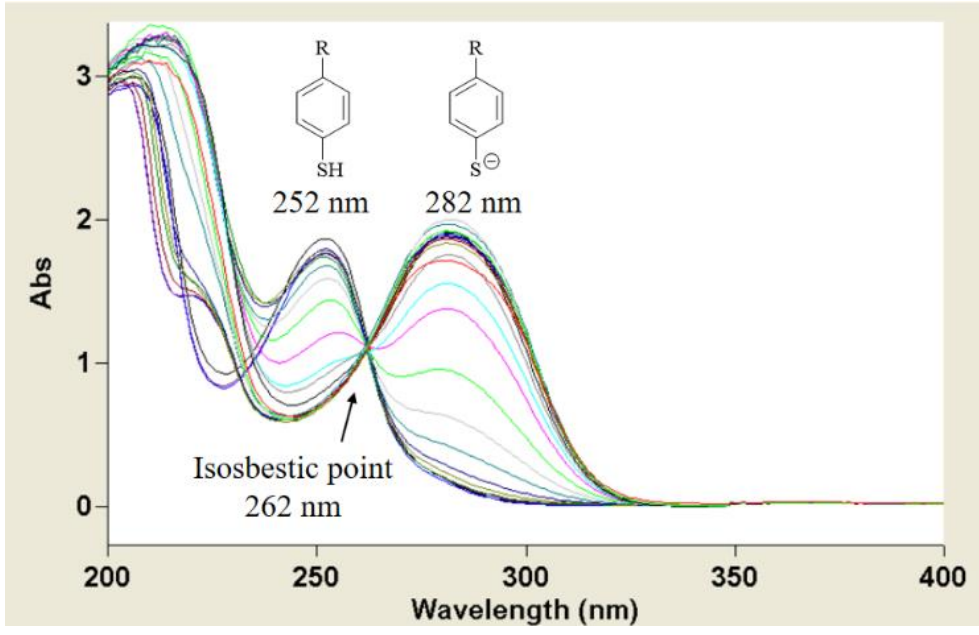

Figure $38 \mathrm{UV}-\mathrm{Vis}$ spectra of hexyl thiol at various $\mathrm{pH}$
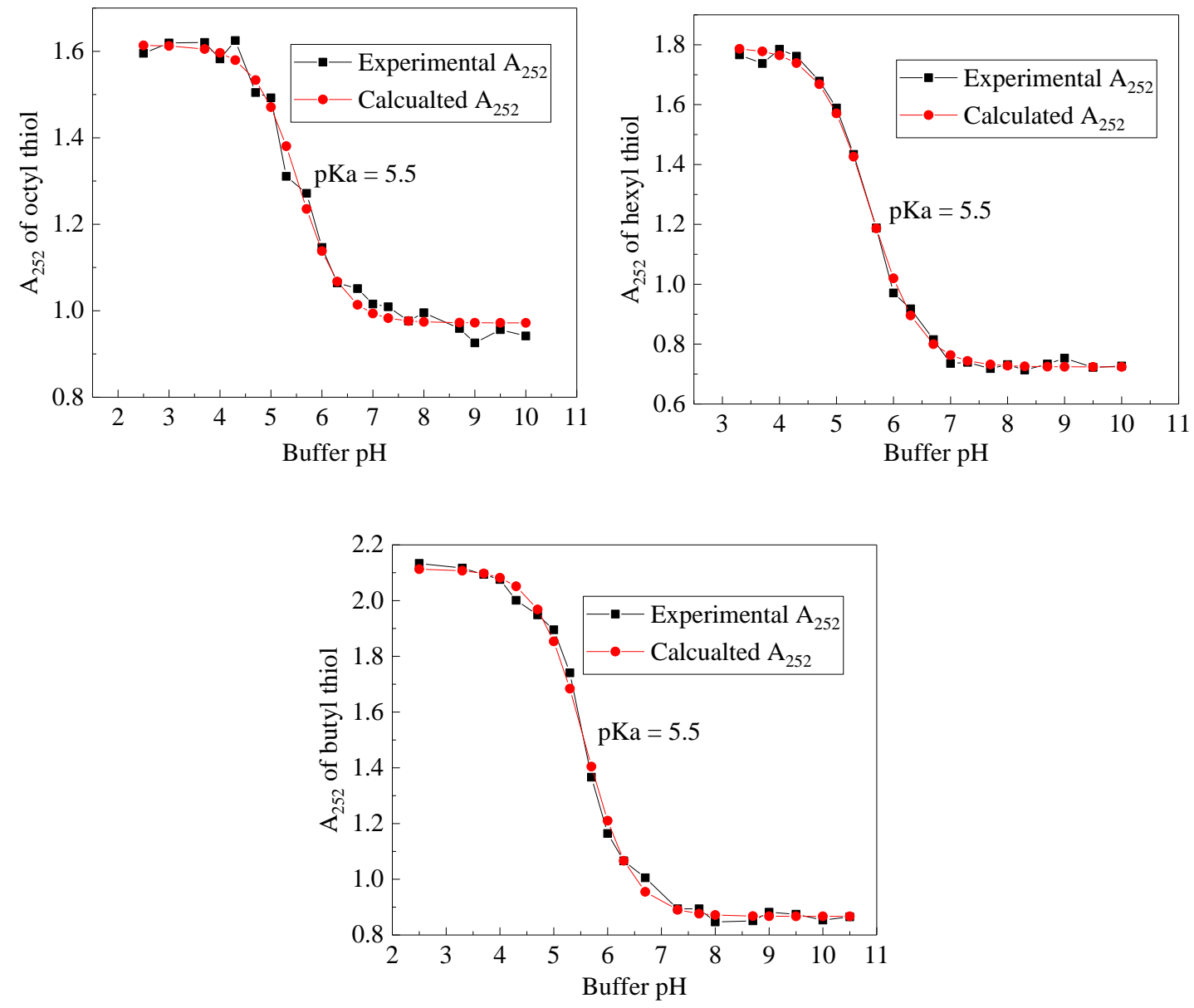

Figure $39 \mathrm{pKa}$ determination of three aromatic thiols 


\subsubsection{Folding of reduced BPTI with octyl thiol and disulfide}

Reduced BPTI was initially folded with different concentrations of octyl disulfide $(0.09,0.125$, and $0.25 \mathrm{mM})$. Folding reactions were quenched at 15, 30, 60, 120, 240 and $480 \mathrm{~min}$. With the increase of octyl disulfide concentration, the folding yield of reduced BPTI was increased. Although about $60 \%$ of native protein was produced in $8 \mathrm{~h}$ with 0.25 $\mathrm{mM}$ octyl disulfide, protein precipitation was observed during the folding process. In the absence of aromatic thiol, low native protein yield was obtained owing to slow thioldisulfide interchange reactions during the folding process. With $0.125 \mathrm{mM}$ octyl disulfide, only about $25 \%$ of native protein was formed after $8 \mathrm{~h}$ (Figure 40 ).

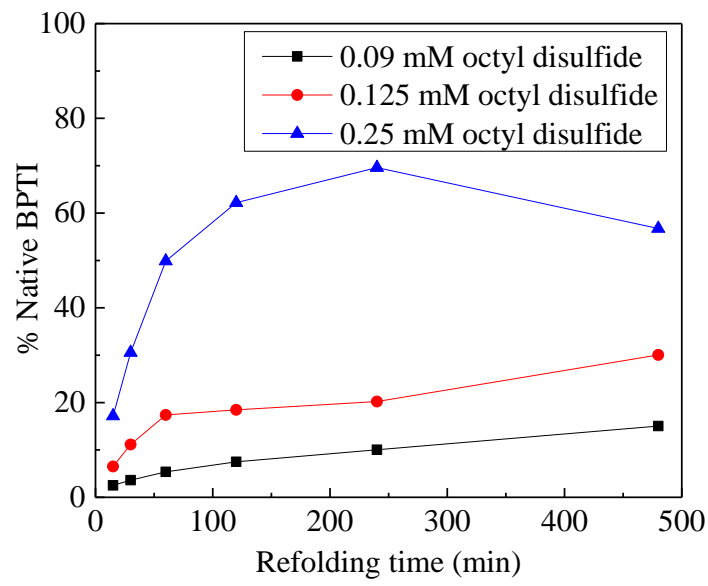

Figure 40 Folding of reduced BPTI with octyl disulfide

Folding of reduced BPTI were conducted in the presence of $0.09 \mathrm{mM}$ octyl disulfide and different concentrations of thiol $(1,2,5$ and $10 \mathrm{mM})$. After $8 \mathrm{~h}$ refolding time, a high percentage of folding intermediates were observed, indicating a low folding yield of native protein (Figure 41). Protein precipitation was observed during the folding with $0.09 \mathrm{mM}$ octyl disulfide and $10 \mathrm{mM}$ thiol. Nonetheless, the folding yield with $0.09 \mathrm{mM}$ octyl disulfide and thiol was increased compared to the folding with only $0.09 \mathrm{mM}$ octyl 
disulfide. With $0.09 \mathrm{mM}$ octyl disulfide and $5 \mathrm{mM}$ thiol, about $66 \%$ of native protein was formed after $8 \mathrm{~h}$ (Figure 42).

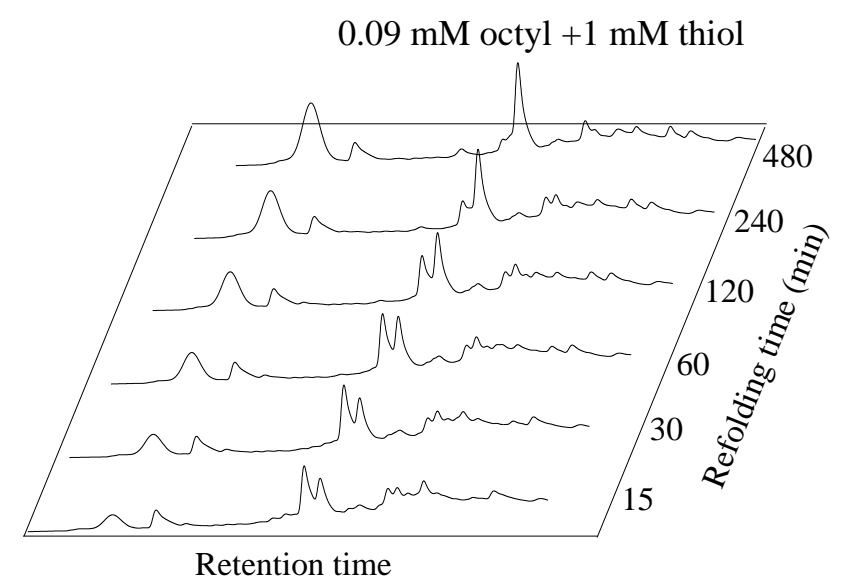

Figure 41 HPLC chromatograms of reduced BPTI folding with octyl disulfide and thiol

In order to increase the native protein yield, octyl disulfide concentration in the redox buffers was increased. Since protein precipitation was observed during the folding of reduced BPTI with only $0.25 \mathrm{mM}$ octyl disulfide, folding reactions were not conducted with $0.25 \mathrm{mM}$ octyl disulfide and thiol. Instead, redox buffers containing $0.125 \mathrm{mM}$ octyl disulfide and different concentrations of octyl thiol (1, 2, 5 and $10 \mathrm{mM})$ were used to fold reduced BPTI. However, the folding yield of native protein showed no significant increase in $8 \mathrm{~h}$. Protein precipitation occurred during the folding with 5 and $10 \mathrm{mM}$ octyl thiol. The folding yield of native protein without precipitation was only about $60 \%$ in $8 \mathrm{~h}$ with 0.125 $\mathrm{mM}$ octyl disulfide and $2 \mathrm{mM}$ thiol.

Compared to QAS thiol (methyl thiol) and its corresponding disulfide, octyl thiol and its disulfide contained a long hydrophobic chain in their structure. With higher concentrations of octyl thiol and disulfide, folding intermediates with mixed disulfide bonds between the protein and the octyl thiol group increased, resulting in more 
hydrophobic interactions as the hydrophobic chain length was increased. These hydrophobic protein folding intermediates formed during the folding process tended to aggregate together in the aqueous solution, leading to the occurrence of protein precipitation.

The folding rate constants and initial folding rates of reduced BPTI with different concentrations of octyl disulfide and thiol were calculated by fitting the results of native protein yield against the refolding time to a single exponential function showing as protein yield $\%=\mathrm{A}\left(1-\mathrm{e}^{-k \mathrm{t}}\right)$. With the increase of octyl thiol and disulfide concentration, the maximal folding yields $(\mathrm{A})$ and the initial folding rates $\left(\mathrm{A}^{*} k\right)$ were increased. The redox buffer containing octyl thiol and disulfide producing the highest yield of native protein without protein precipitation was determined to be $0.09 \mathrm{mM}$ octyl disulfide and $5 \mathrm{mM}$ thiol. The maximal folding yield of native protein with $0.09 \mathrm{mM}$ octyl disulfide and $5 \mathrm{mM}$ thiol was calculated to be $62 \pm 4 \%$, and the initial folding rate was 1.40 $\pm 0.31 \% / \mathrm{min}($ Table 5$)$.
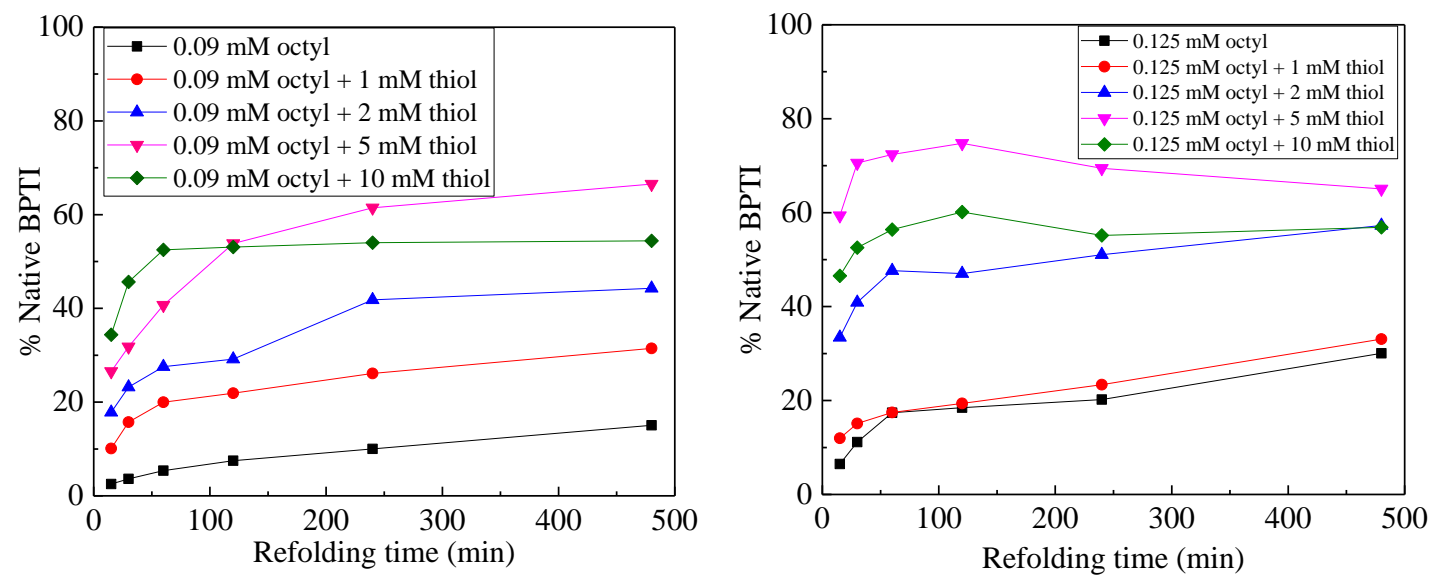

Figure 42 Folding of reduced BPTI with octyl disulfide and thiol 
Table 5 Folding of reduced BPTI with octyl disulifde and thiol

\begin{tabular}{ccccc}
\hline $\begin{array}{c}\text { Redox buffers } \\
\text { Octyl disulfide } \\
(\mathrm{mM})\end{array}$ & $\begin{array}{c}\text { Octyl thiol } \\
(\mathrm{mM})\end{array}$ & $\begin{array}{c}\text { A } \\
(\% \text { native protein })\end{array}$ & $\begin{array}{c}k \\
\left(\mathrm{~min}^{-1}\right)\end{array}$ & $\begin{array}{c}\text { Initial rate } \\
(\% / \mathrm{min})\end{array}$ \\
\hline & 0 & $15 \pm 2$ & $0.006 \pm 0.002$ & $0.09 \pm 0.03$ \\
$\mathbf{0 . 0 9}$ & 1 & $27 \pm 2$ & $0.025 \pm 0.006$ & $0.67 \pm 0.18$ \\
& 2 & $39 \pm 4$ & $0.025 \pm 0.008$ & $0.99 \pm 0.35$ \\
& $\mathbf{5}$ & $\mathbf{6 2} \pm \mathbf{4}$ & $\mathbf{0 . 0 2 3} \pm \mathbf{0 . 0 0 5}$ & $\mathbf{1 . 4 0} \pm \mathbf{0 . 3 1}$ \\
& $10^{*}$ & $54 \pm 0.3$ & $0.066 \pm 0.002$ & $3.56 \pm 0.11$ \\
\hline & 0 & $25 \pm 3$ & $0.017 \pm 0.006$ & $0.42 \pm 0.16$ \\
& 1 & $26 \pm 3$ & $0.024 \pm 0.011$ & $0.62 \pm 0.29$ \\
& 2 & $51 \pm 2$ & $0.063 \pm 0.013$ & $3.20 \pm 0.67$ \\
& $5 *$ & $71 \pm 2$ & $0.125 \pm 0.025$ & $8.84 \pm 1.77$ \\
& $10 *$ & $57 \pm 1$ & $0.109 \pm 0.014$ & $6.18 \pm 0.80$ \\
\hline 0.25 & $0 *$ & $64 \pm 3$ & $0.023 \pm 0.004$ & $1.49 \pm 0.27$ \\
\hline
\end{tabular}

*Protein precipitation occurred during folding.

\subsubsection{Folding of reduced BPTI with hexyl thiol and disulfide}

Compared to octyl thiol, hexyl thiol with a shorter alkyl group was expected to minimize protein precipitation that occurred during folding. To detect the effects of concentration on folding, different mixtures of hexyl disulfide $(0.09,0.25$ and $0.5 \mathrm{mM})$ were examined in the folding reactions (Figure 43). At 5, 30, 60, 240, 480 and 1440 min time points, aliquots of the reaction mixture were quenched with formic acid. No protein precipitation was observed during folding. With $0.09 \mathrm{mM}$ hexyl disulfide, only about $27 \%$ native protein was obtained in $24 \mathrm{~h}$. As the hexyl disulfide concentration rose to 0.25 and $0.5 \mathrm{mM}$, about $80 \%$ of native protein was produced in $4 \mathrm{~h}$ and the yield remained the same at $24 \mathrm{~h}$ owning to the lack of reducing agents that could catalyze the rearrangement of mixed disulfide folding intermediates.

Folding reactions of reduced BPTI were then conducted with $0.09 \mathrm{mM}$ hexyl disulfide and different concentrations of hexyl thiol $(1,2,5$ and $10 \mathrm{mM})$. Aliquots of the reaction 
mixtures were quenched at 5, 15, 30, 60, 120 and $240 \mathrm{~min}$. Higher folding yields of native BPTI were achieved compared to the folding with only hexyl disulfide in the redox buffers. HPLC chromatograms showed that the rates of native protein formation and folding intermediate rearrangement were increased with higher concentration of hexyl thiol (Figure 44). The folding of reduced BPTI was completed in $2 \mathrm{~h}$ in terms of the formation of about $90 \%$ of native protein in the presence of $0.09 \mathrm{mM}$ hexyl disulfide and $10 \mathrm{mM}$ thiol (Figure 45). The initial folding rate reached $9.3 \pm 1.1 \% / \mathrm{min}$ when reduced BPTI was folded with $0.09 \mathrm{mM}$ hexyl disulfide and $10 \mathrm{mM}$ thiol (Table 6).

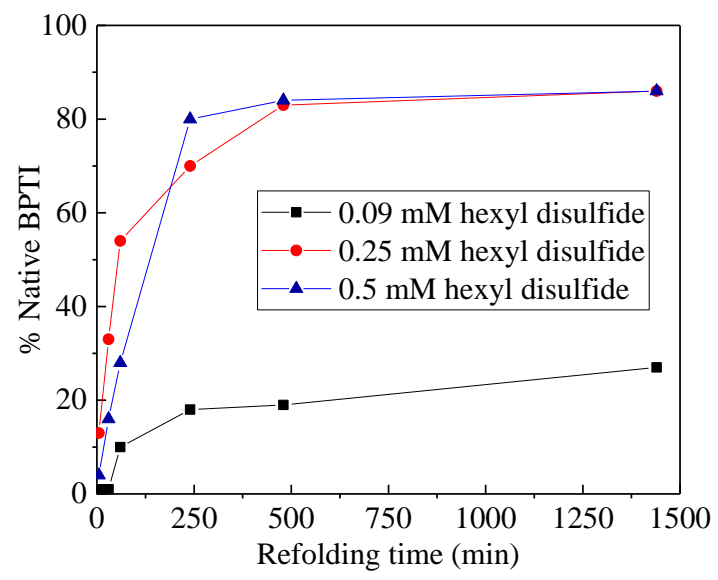

Figure 43 Folding of reduced BPTI with hexyl disulfide

Folding of reduced BPTI with $0.25 \mathrm{mM}$ hexyl disulfide and different concentrations of hexyl thiol (1, 2, 5 and $10 \mathrm{mM}$ ) showed higher folding yields in $4 \mathrm{~h}$ (Figure 45). About $90 \%$ of native BPTI was obtained at all thiol concentrations, and the folding process was completed in $1 \mathrm{~h}$ with $0.25 \mathrm{mM}$ hexyl disulfide, and 5 and $10 \mathrm{mM}$ hexyl thiol. Compared to the folding with $0.09 \mathrm{mM}$ hexyl disulfide and thiol, the folding rates and native protein yields were increased with $0.25 \mathrm{mM}$ hexyl disulfide and the same concentration of thiol. With curve fitting calculations, the maximal folding yield of reduced BPTI was $90 \pm 1 \%$, 
and the initial folding rate was $9.42 \pm 0.54 \% /$ min when reduced BPTI was folded with $0.25 \mathrm{mM}$ hexyl disulfide and $10 \mathrm{mM}$ thiol (Table 6).

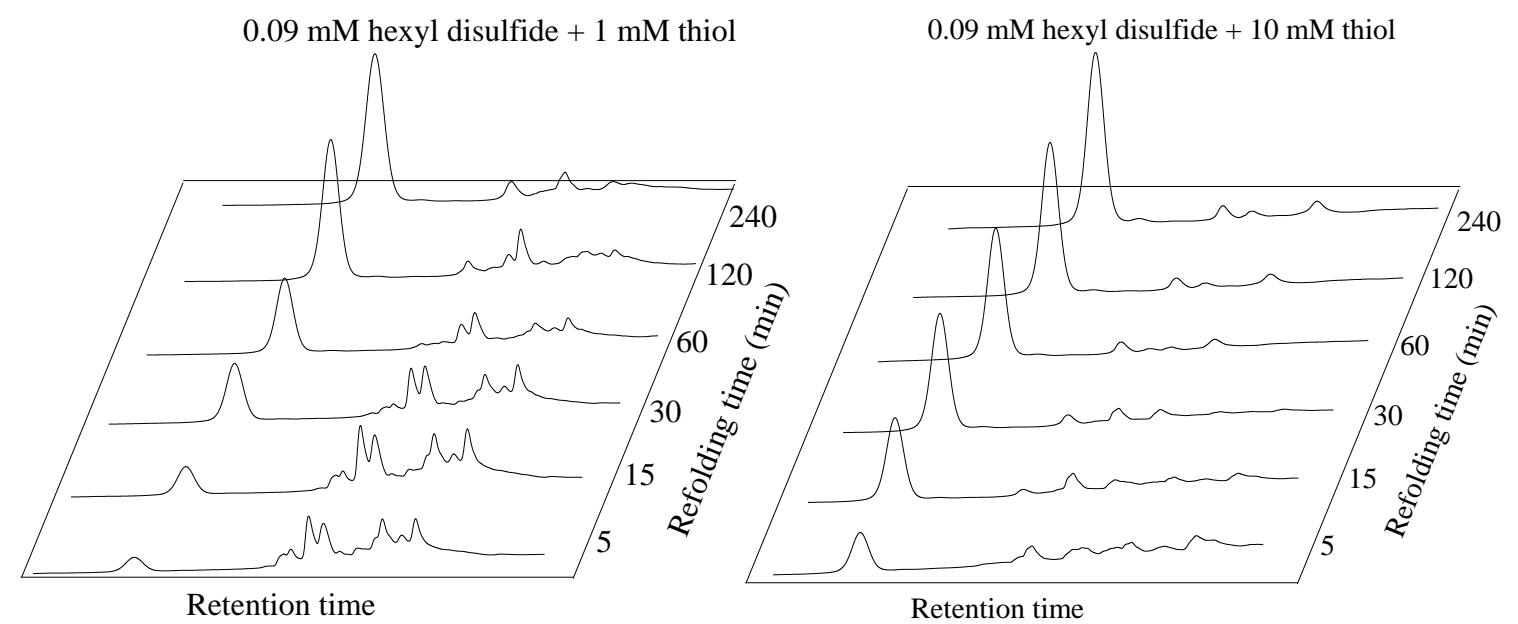

Figure 44 HPLC chromatograms of reduced BPTI with hexyl disulfide and thiol

Table 6 Folding of reduced BPTI with hexyl disulfide and thiol

\begin{tabular}{|c|c|c|c|c|}
\hline \multicolumn{2}{|c|}{ Redox buffers } & \multirow[b]{2}{*}{$\begin{array}{c}\mathrm{A} \\
\text { (\%native protein) }\end{array}$} & \multirow[b]{2}{*}{$\begin{array}{c}k \\
\left(\min ^{-1}\right)\end{array}$} & \multirow[b]{2}{*}{$\begin{array}{c}\text { Initial rate } \\
(\% / \mathrm{min})\end{array}$} \\
\hline $\begin{array}{l}\text { Hexyl disulfide } \\
(\mathrm{mM})\end{array}$ & $\begin{array}{l}\text { Hexyl thiol } \\
(\mathrm{mM})\end{array}$ & & & \\
\hline \multirow{4}{*}{0.09} & 1 & $77 \pm 3$ & $0.017 \pm 0.002$ & $1.34 \pm 0.14$ \\
\hline & 2 & $85 \pm 1$ & $0.032 \pm 0.001$ & $2.73 \pm 0.11$ \\
\hline & 5 & $85 \pm 3$ & $0.077 \pm 0.010$ & $6.57 \pm 0.85$ \\
\hline & 10 & $88 \pm 2$ & $0.106 \pm 0.012$ & $9.29 \pm 1.11$ \\
\hline \multirow{4}{*}{0.25} & 1 & $88 \pm 3$ & $0.020 \pm 0.002$ & $1.78 \pm 0.18$ \\
\hline & 2 & $92 \pm 2$ & $0.040 \pm 0.001$ & $3.63 \pm 0.34$ \\
\hline & 5 & $91 \pm 1$ & $0.066 \pm 0.010$ & $5.97 \pm 0.23$ \\
\hline & 10 & $90 \pm 1$ & $0.105 \pm 0.012$ & $9.42 \pm 0.54$ \\
\hline \multirow{4}{*}{1} & 1 & $106 \pm 4$ & $0.008 \pm 0.001$ & $0.84 \pm 0.07$ \\
\hline & 2 & $95 \pm 3$ & $0.016 \pm 0.001$ & $1.49 \pm 0.12$ \\
\hline & $5 *$ & $94 \pm 1$ & $0.039 \pm 0.002$ & $3.73 \pm 0.16$ \\
\hline & $10 *$ & $95 \pm 1$ & $0.054 \pm 0.003$ & $5.14 \pm 0.26$ \\
\hline \multirow{4}{*}{2.5} & $1 *$ & $127 \pm 8$ & $0.003 \pm 0.003$ & $0.42 \pm 0.05$ \\
\hline & $2 *$ & $100 \pm 4$ & $0.008 \pm 0.008$ & $0.79 \pm 0.06$ \\
\hline & $5^{*}$ & $98 \pm 4$ & $0.017 \pm 0.017$ & $1.69 \pm 0.18$ \\
\hline & $10^{*}$ & $98 \pm 2$ & $0.024 \pm 0.001$ & $2.40 \pm 0.12$ \\
\hline
\end{tabular}

*Protein precipitation occurred during folding. 

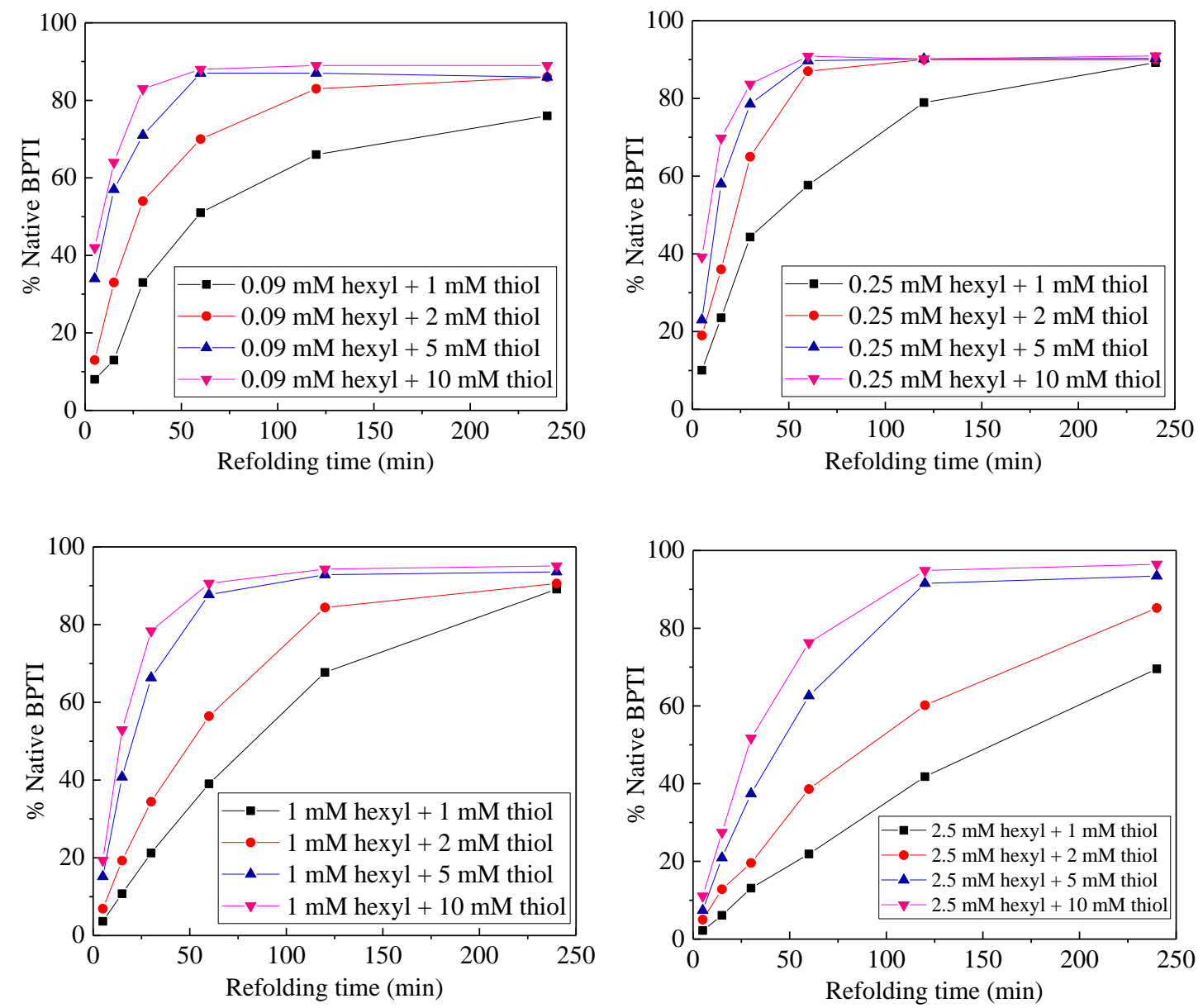

Figure 45 Folding of reduced BPTI with hexyl disulfide and thiol

When reduced BPTI was folded with $1 \mathrm{mM}$ hexyl disulfide and different concentrations of thiol $(1,2,5$ and $10 \mathrm{mM})$, protein precipitation was observed with $1 \mathrm{mM}$ hexyl disulfide, and 5 and $10 \mathrm{mM}$ thiol. The folding yields of native protein were calculated using the soluble form of the protein after folding reactions. About $90 \%$ of native protein was produced in $4 \mathrm{~h}$ with $1 \mathrm{mM}$ hexyl disulfide and $2 \mathrm{mM}$ thiol. The maximal folding yield of native protein was $95 \pm 3 \%$, and the initial folding rate was determined to be $1.49 \pm$ $0.12 \% / \mathrm{min}$. The initial folding rates were decreased with $1 \mathrm{mM}$ hexyl disulfide and different concentrations of hexyl thiol compared to the rates with lower hexyl disulfide concentrations. 
When the hexyl disulfide concentration in the redox buffers was increased to $2.5 \mathrm{mM}$, protein precipitation occurred at all thiol concentrations. High concentrations of hexyl thiol and disulfide tend to form more hydrophobic folding intermediates during folding, leading to a higher propensity of protein precipitation. The folding process of reduced BPTI with $2.5 \mathrm{mM}$ hexyl disulfide, and 1 and $2 \mathrm{mM}$ thiol was not completed in $4 \mathrm{~h}$ due to a high concentration ratio of hexyl disulfide and thiol, which led to slow thiol-disulfide rearrangement during folding. When the folding results of reduced BPTI with $2.5 \mathrm{mM}$ disulfide and thiol were fitted to the exponential function, high errors were shown with low thiol concentrations. All initial folding rates with $2.5 \mathrm{mM}$ hexyl disulfide and thiol were determined to be lower than the rates with other hexyl disulfide concentrations and the same thiol concentration. With $2.5 \mathrm{mM}$ hexyl disulfide and $10 \mathrm{mM}$ thiol, the initial folding rate was $2.40 \pm 0.12 \% / \mathrm{min}$ compared to $9.42 \pm 0.54 \% / \mathrm{min}$ with $0.25 \mathrm{mM}$ hexyl disulfide and $10 \mathrm{mM}$ thiol.

The folding yields of native BPTI with $2 \mathrm{mM}$ hexyl thiol and different concentrations of disulfide $(0.09,0.25,1$ and $2.5 \mathrm{mM})$ were then plotted against the refolding time (Figure 46). Reduced BPTI folding with $0.25 \mathrm{mM}$ hexyl disulfide obtained fastest folding, showing that about $90 \%$ of native protein was produced in $2 \mathrm{~h}$. The folding rates were increased when changing the disulfide concentration from $0.09 \mathrm{mM}$ to $0.25 \mathrm{mM}$, while the rate decreased when the disulfide concentration was further increased to $1 \mathrm{mM}$ and $2.5 \mathrm{mM}$ (Figure 47). Therefore, $0.25 \mathrm{mM}$ hexyl disulfide was determined to be the optimal disulfide concentration to fold reduced BPTI effectively. 


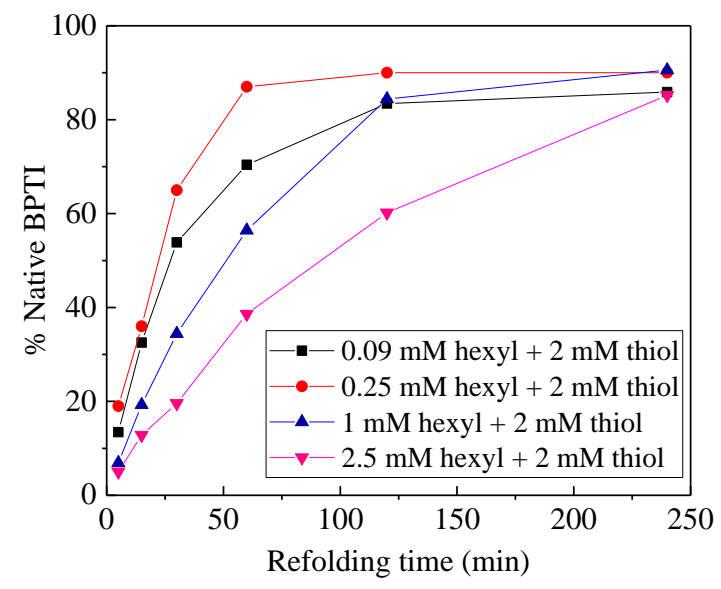

Figure 46 Folding of reduced BPTI with $2 \mathrm{mM}$ hexyl thiol and disulfide

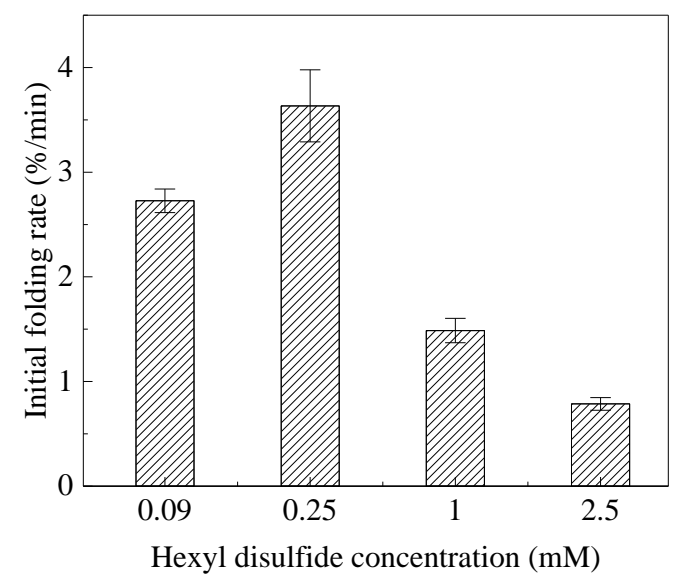

Figure 47 Initial folding rates of reduced BPTI with $2 \mathrm{mM}$ hexyl thiol and disulfide

\subsubsection{Folding of reduced BPTI with butyl thiol and disulfide}

Redox buffers containing different concentration combinations of butyl disulfide ( 0.09 , $0.25,1$ and $2.5 \mathrm{mM})$ and its corresponding thiol $(1,2,5$ and $10 \mathrm{mM})$ were used to fold reduced BPTI in vitro. No protein precipitation was observed at all folding conditions with butyl thiol and disulfides, which is likely due to the fact that butyl thiol has a shorter hydrocarbon chain on the aromatic ring compared to hexyl and octyl thiols.

Surprisingly, folding with butyl disulfide and thiol produced less than $90 \%$ native protein (Figure 48). When reduced BPTI was folded with $0.09 \mathrm{mM}$ butyl disulfide and 
thiol, the native protein yield was increased with increasing concentrations of butyl thiol. With $0.09 \mathrm{mM}$ butyl disulfide and $10 \mathrm{mM}$ thiol, about $83 \%$ of native protein was produced in $1 \mathrm{~h}$ and the folding yield remained the same at $4 \mathrm{~h}$. The folding data of native protein yield against the refolding time was fitted to the single exponential function. When reduced BPTI was folded with $0.09 \mathrm{mM}$ butyl disulfide and $10 \mathrm{mM}$ thiol, the maximal folding yield was calculated to be $81 \pm 2 \%$, and the initial folding rate was $3.07 \pm 0.21 \% / \mathrm{min}$ (Table 7).
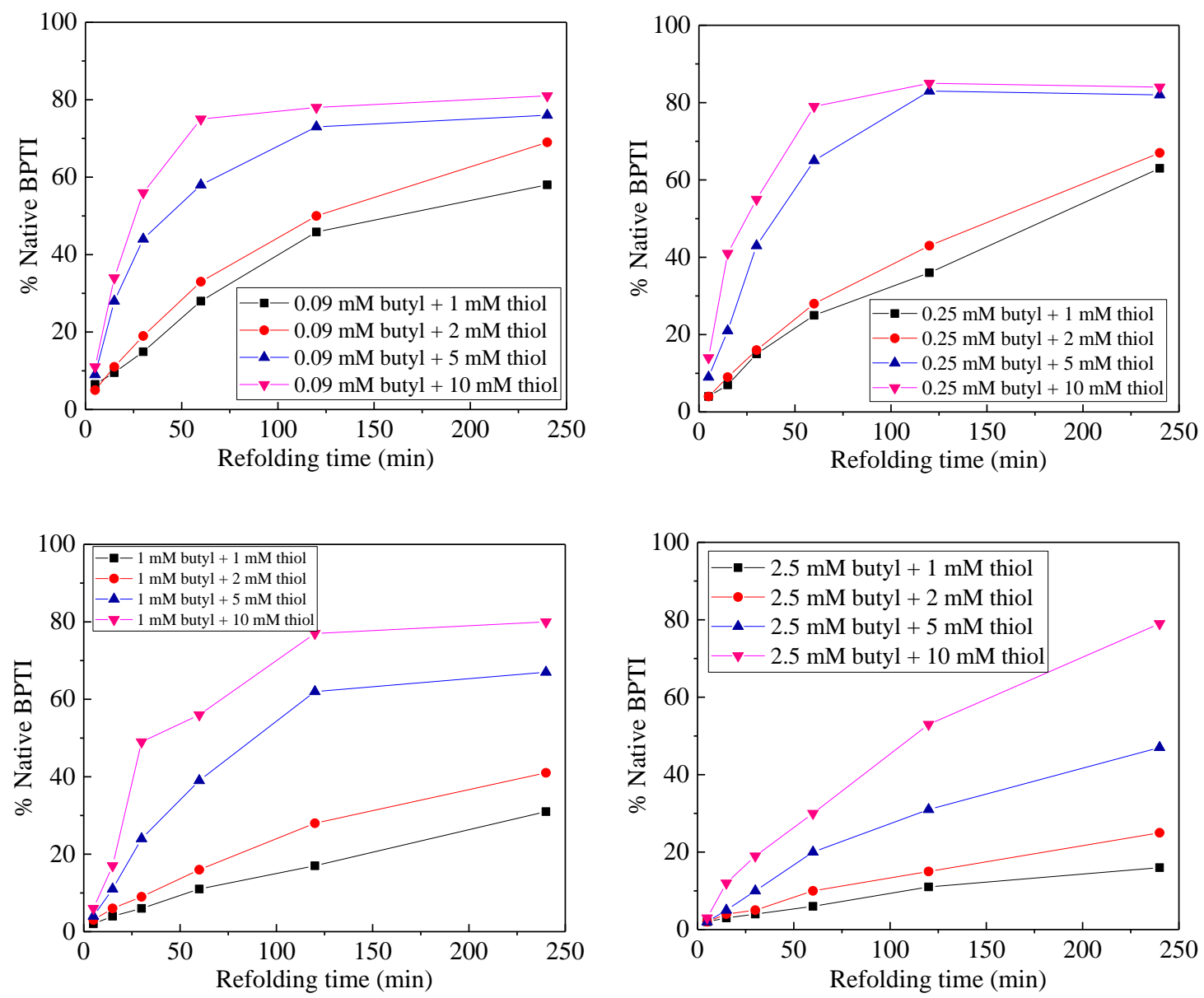

Figure 48 Folding of reduced BPTI with butyl disulfide and thiol 
Table 7 Folding of reduced BPTI with butyl disulfide and thiol

\begin{tabular}{|c|c|c|c|c|}
\hline \multicolumn{2}{|c|}{ Redox buffers } & \multirow[b]{2}{*}{$\begin{array}{c}\mathrm{A} \\
\text { (\%native protein) }\end{array}$} & \multirow[b]{2}{*}{$\begin{array}{c}k \\
\left(\min ^{-1}\right)\end{array}$} & \multirow[b]{2}{*}{$\begin{array}{c}\text { Initial rate } \\
(\% / \mathrm{min})\end{array}$} \\
\hline $\begin{array}{l}\text { Butyl disulfide } \\
(\mathrm{mM})\end{array}$ & $\begin{array}{l}\text { Butyl thiol } \\
(\mathrm{mM})\end{array}$ & & & \\
\hline \multirow{4}{*}{0.09} & 1 & $64 \pm 4$ & $0.010 \pm 0.001$ & $0.63 \pm 0.08$ \\
\hline & 2 & $77 \pm 2$ & $0.009 \pm 0.001$ & $0.71 \pm 0.05$ \\
\hline & 5 & $75 \pm 2$ & $0.028 \pm 0.002$ & $2.11 \pm 0.15$ \\
\hline & 10 & $81 \pm 2$ & $0.038 \pm 0.003$ & $3.07 \pm 0.21$ \\
\hline \multirow{4}{*}{0.25} & 1 & $89 \pm 15$ & $0.005 \pm 0.001$ & $0.44 \pm 0.14$ \\
\hline & 2 & $84 \pm 6$ & $0.006 \pm 0.001$ & $0.54 \pm 0.08$ \\
\hline & 5 & $85 \pm 3$ & $0.023 \pm 0.002$ & $1.97 \pm 0.18$ \\
\hline & 10 & $85 \pm 2$ & $0.039 \pm 0.003$ & $3.33 \pm 0.27$ \\
\hline \multirow{4}{*}{1} & 1 & $54 \pm 12$ & $0.004 \pm 0.001$ & $0.19 \pm 0.08$ \\
\hline & 2 & $52 \pm 3$ & $0.006 \pm 0.001$ & $0.33 \pm 0.04$ \\
\hline & 5 & $72 \pm 3$ & $0.014 \pm 0.002$ & $0.99 \pm 0.12$ \\
\hline & 10 & $81 \pm 5$ & $0.023 \pm 0.004$ & $1.85 \pm 0.37$ \\
\hline \multirow{4}{*}{2.5} & 1 & $20 \pm 3$ & $0.007 \pm 0.002$ & $0.14 \pm 0.04$ \\
\hline & 2 & $34 \pm 5$ & $0.005 \pm 0.001$ & $0.18 \pm 0.05$ \\
\hline & 5 & $60 \pm 3$ & $0.006 \pm 0.001$ & $0.38 \pm 0.03$ \\
\hline & 10 & $101 \pm 7$ & $0.006 \pm 0.001$ & $0.63 \pm 0.09$ \\
\hline
\end{tabular}

When the folding of reduced BPTI was conducted with $0.25 \mathrm{mM}$ butyl disulfide, and 5 and $10 \mathrm{mM}$ butyl thiol, native protein yield was about $85 \%$ after $2 \mathrm{~h}$, which was a slight increase compared to folding with $0.09 \mathrm{mM}$ butyl disulfide and the same thiol concentrations. The initial folding rate of reduced BPTI with $0.25 \mathrm{mM}$ butyl disulfide and $10 \mathrm{mM}$ thiol was $3.33 \pm 0.27 \% / \mathrm{min}$. With $0.25 \mathrm{mM}$ butyl disulfide and lower thiol concentration, 1 and $2 \mathrm{mM}$, the folding of reduced BPTI was not completed as only about $65 \%$ of native protein was produced in $4 \mathrm{~h}$.

Low native protein yields were obtained with higher concentrations of butyl disulfide (1 and $2.5 \mathrm{mM}$ ) and different concentrations of butyl thiol, and the folding process was not completed in $4 \mathrm{~h}$. At the same butyl disulfide concentration, the folding yield of native protein was increased with increasing butyl thiol concentrations in the redox buffer. When 
reduced BPTI was folded with redox buffers containing $1 \mathrm{mM}$ butyl disulfide and thiol, the folding yield was about $80 \%$ with $10 \mathrm{mM}$ butyl thiol in 4 h relative to $30 \%$ with $1 \mathrm{mM}$ thiol. Folding of reduced BPTI with $2.5 \mathrm{mM}$ butyl disulfide and thiol was not completed and the initial folding rates at all conditions were lower than the rates with $1 \mathrm{mM}$ butyl disulfide and the same concentration of thiol.

During the folding of reduced BPTI with butyl thiol and disulfide, a kinetically stable intermediate was produced at all folding conditions (Figure 49). With $0.09 \mathrm{mM}$ butyl disulfide and $10 \mathrm{mM}$ thiol, about $10 \%$ of the intermediate still remained after $4 \mathrm{~h}$, leading to a native protein yield of lower than $90 \%$ (Figure 50). With a high concentration ratio of butyl disulfide and thiol, [RSSR]:[RSH], the rearrangement of the folding intermediate to native protein through thiol-disulfide interchange was slow. With $1 \mathrm{mM}$ butyl disulfide and $1 \mathrm{mM}$ thiol, about $24 \%$ of the folding intermediate was produced after $4 \mathrm{~h}$ refolding time. In comparison, when the thiol concentration was increased to $10 \mathrm{mM}$, about $8 \%$ of the folding intermediate was accumulated within $4 \mathrm{~h}$.

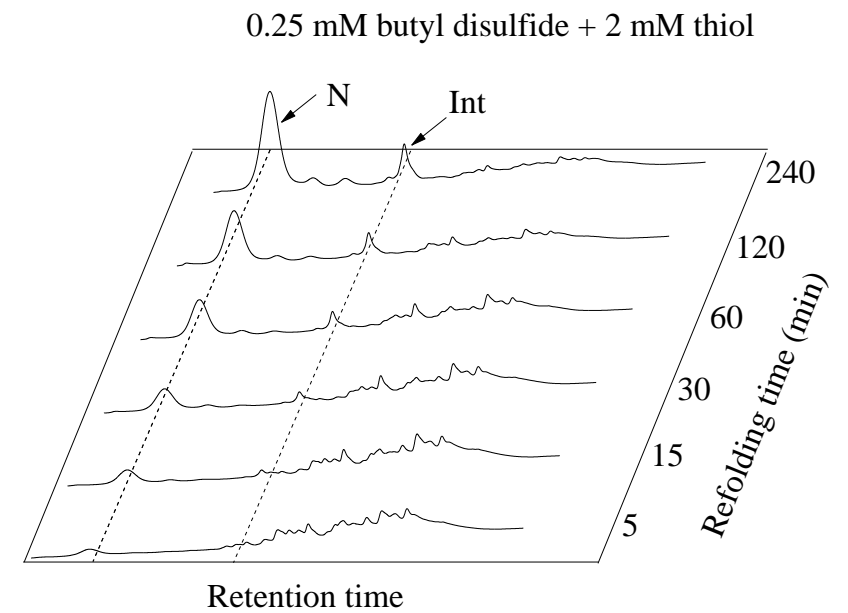

Figure 49 HPLC chromatograms of reduced BPTI folding with butyl disulfide and thiol 

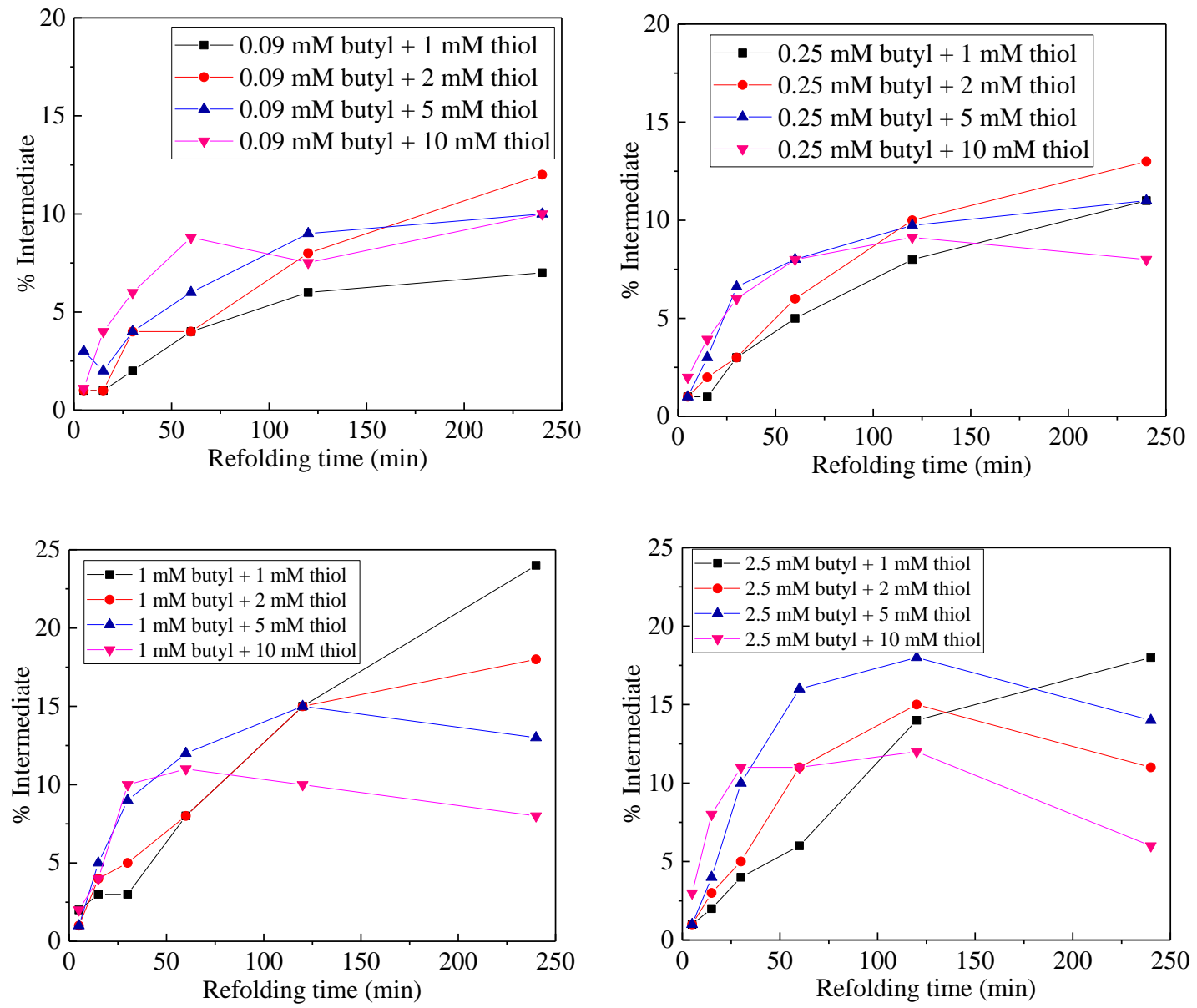

Figure 50 Folding intermediate formation of reduced BPTI with butyl disulfide and thiol

In summary, high yields of native protein yield were not obtained during folding with butyl disulfide and thiol owing to the formation of a prominent kinetically stable intermediate at all folding conditions. The best folding concentration of butyl thiol and disulfide to fold reduced BPTI was determined to be $0.25 \mathrm{mM}$ butyl disulfide and $10 \mathrm{mM}$ thiol, which produced $85 \%$ of native protein in $2 \mathrm{~h}$ folding time, which was the fastest initial folding rate when compared to other butyl thiol and disulfide mixtures.

\subsection{Conclusion}

Three aromatic thiols, including octyl, hexyl and butyl thiols, and their corresponding disulfides were used as redox buffers to fold reduced BPTI in vitro. All three aromatic 
thiols had an elongated hydrocarbon chain in the structure, which were more hydrophobic than QAS thiol (methyl thiol). Compared to traditional redox buffers such as GSH/GSH, all aromatic thiols and their corresponding disulfides in the study facilitated the folding of reduced BPTI (Figure 51). As stated in a previous chapter, more than $90 \%$ of native BPTI was produced in $2 \mathrm{~h}$ with $0.25 \mathrm{mM}$ QAS disulfide and $10 \mathrm{mM}$ QAS thiol, and only about $25 \%$ of native protein was obtained with $5 \mathrm{mM} \mathrm{GSSG}$ and $5 \mathrm{mM} \mathrm{GSH}$. Within $4 \mathrm{~h}$ refolding time, about $66 \%$ of native BPTI was produced using the optimal concentration of octyl disulfide and thiol (0.09 $\mathrm{mM}$ octyl disulfide and $5 \mathrm{mM}$ octyl thiol). At $0.25 \mathrm{mM}$ butyl disulfide and $10 \mathrm{mM}$ butyl thiol, about $85 \%$ of native protein was obtained in $2 \mathrm{~h}$. More importantly, more than $90 \%$ of native protein was produced in $1 \mathrm{~h}$ with the optimal condition of hexyl disulfide and thiol (0.25 mM hexyl disulfide and $10 \mathrm{mM}$ hexyl thiol).

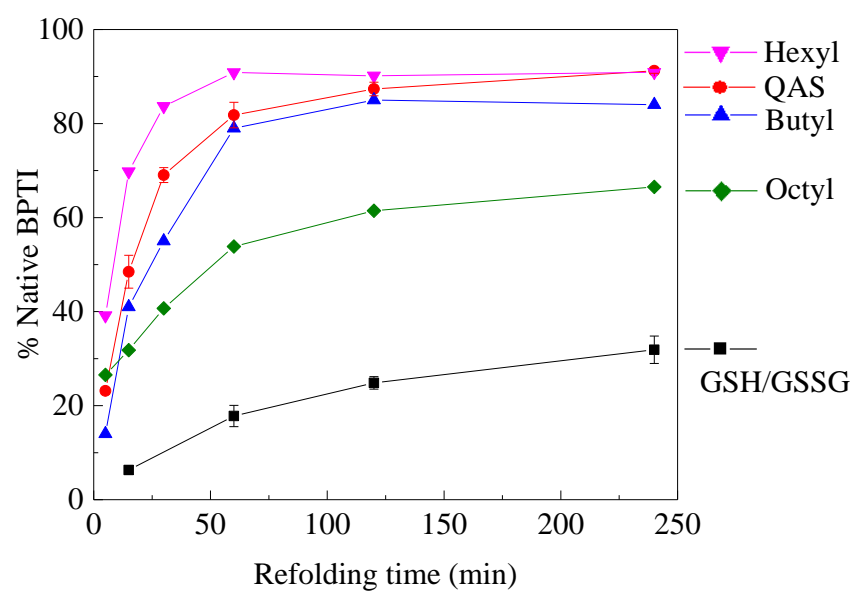

Figure 51 Reduced BPTI folding with aromatic thiols/disulfides and GSH/GSSG

The initial folding rates of reduced BPTI with redox buffers containing the optimal combinations of each thiol and its corresponding disulfide were calculated by the curve fitting to the exponential function. Aromatic thiols and disulfides significantly increased the initial folding rates of the reaction. Folding of reduced BPTI with hexyl thiol and 
disulfide obtained the highest initial folding rate, which was $9.42 \pm 0.54 \% / \mathrm{min}$ (Figure 52).

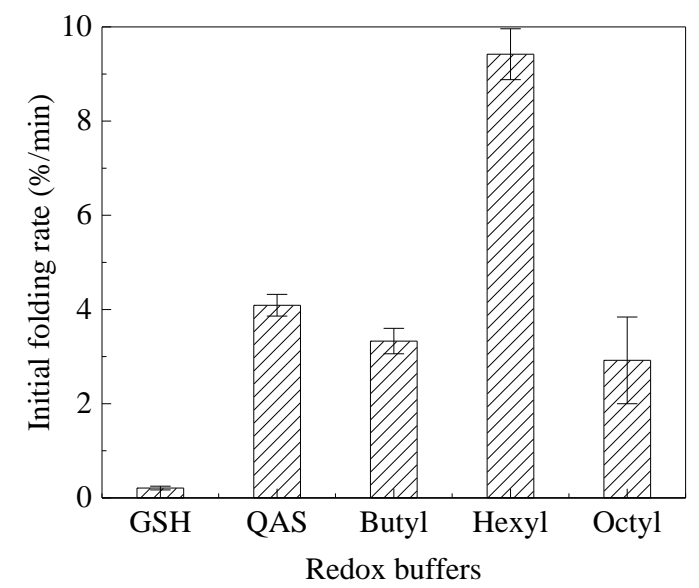

Figure 52 The effect of n-alkyl chain length of small molecule thiols and disulfides on the folding of reduced BPTI

The formation of kinetically stable folding intermediates affected the native protein yields of reduced BPTI folding with different small molecule thiols and disulfides (Figure 53). During the folding of reduced BPTI with traditional redox buffer containing GSSG/GSH, large amounts of kinetically stable intermediates, $\mathrm{N}^{\prime}$ and $\mathrm{N}^{*}$ were accumulated. For both QAS and hexyl thiols, more than $90 \%$ of native protein was obtained and only about $4 \%$ of a kinetically stable folding intermediate was observed after $4 \mathrm{~h}$. When reduced BPTI was folded with the best redox buffer condition of butyl thiol and disulfide, $8 \%$ of a folding intermediate still remained after $4 \mathrm{~h}$ leading to diminished yield of native protein. 


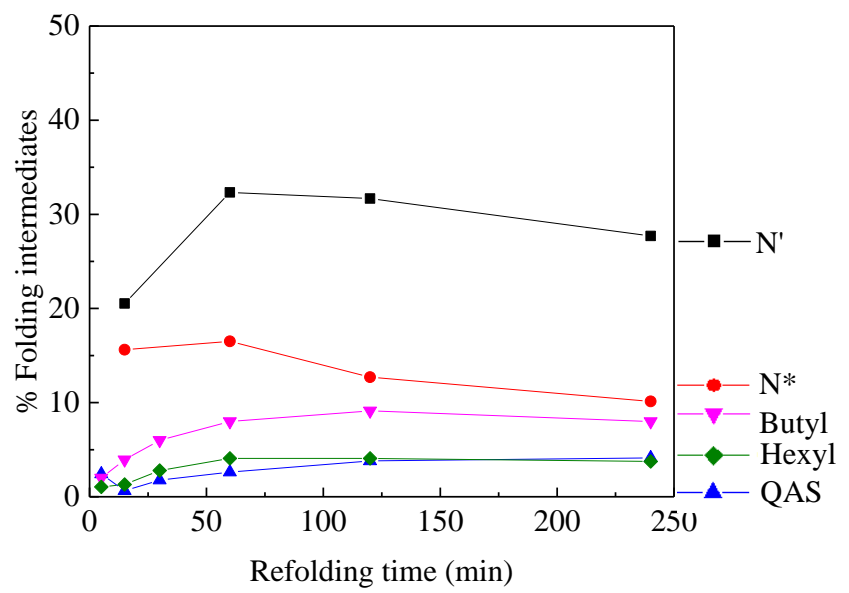

Figure 53 Folding intermdiates of reduced BPTI with aromatic thiols and disulfides, and GSH/GSSG $\left(\mathrm{N}^{\prime}\right.$ and $\left.\mathrm{N}^{*}\right)$

When the folding of reduced BPTI was conducted with high concentrations of octyl and hexyl thiols and the corresponding disulfide respectively, protein precipitation was observed owing to the higher hydrophobicity of mixed disulfide intermediates during the folding process. The longer the hydrocarbon chain in the aromatic thiol structure and the higher concentration of aromatic thiol and disulfide, the greater the propensity for protein precipitation was to occur. With all redox buffer conditions tested in the folding study, no protein precipitation was observed at all concentration combinations of butyl thiol and its disulfide. However, protein precipitation was observed when folding with $1 \mathrm{mM}$ hexyl disulfide and $5 \mathrm{mM}$ hexyl thiol or higher redox buffer concentration. When reduced BPTI was folded with octyl thiol and disulfide, more than $0.25 \mathrm{mM}$ octyl disulfide would lead to the occurrence of protein precipitation. 


\section{Oxidative folding of reduced BPTI using aromatic thiols with varying pKa values}

\subsection{Abstract}

Aromatic thiols and disulfides have been used to improve the folding rates and yields of many disulfide-containing proteins including RNase A and BPTI. Aromatic thiols having different $\mathrm{pKa}$ values will have a different distribution of thiol and thiolate ions at a given folding $\mathrm{pH}$, and only the thiolate form of the thiol is the active nucleophile during the nucleophilic attack in a thiol-disulfide interchange reaction. Understanding how thiol pKa values of aromatic thiols affect the folding efficiency of disulfide-containing proteins is important in protein folding studies. An extended thiol, designed based on the QAS thiol structure, was determined to have a a higher thiol pKa value. Folding of reduced BPTI was conducted with different concentrations of extended thiol and its corresponding disulfide.

\subsection{Introduction}

Aromatic thiols and disulfides have been shown to increase the folding rate and yield of disulfide-containing proteins compared to aliphatic thiols and disulfides such as GSH and GSSG. Thiol-disulfide interchange reactions are considered to be the rate-limiting steps during the folding process of disulfide-containing proteins with small molecule thiols and disulfides. In thiol-disulfide interchange reactions, the overall reaction rate is affected by the nucleophilicity of the attacking sulfur, electrophilicity of the center atom, and the stability of the leaving group. The active form of the thiol as a nucleophile is the thiolate, and the thiolate concentration is related to the thiol $\mathrm{pKa}$ value and the folding $\mathrm{pH}$. Aromatic thiols have lower pKa values $(\mathrm{pKa}=4-7)$ than GSH with a thiol pKa value of 8.7. Aromatic thiols possess high nucleophilic ability at the folding $\mathrm{pH}$ of 7.3 as almost all thiols are in 
the active thiolate form. In addition, aromatic thiols with lower thiol $\mathrm{pKa}$ values make the corresponding disulfides more reactive, which can facilitate the formation of mixed disulfide bonds between the protein thiol group and the aromatic thiol. Therefore, an optimal thiol $\mathrm{pKa}$ value of aromatic thiols needs to be determined in order to maintain faster folding rates of the reaction as well as form fewer mixed disulfide intermediates during folding.

An extended thiol was predicted to have a higher thiol pKa than that of QAS thiol, but still lower than the pKa of GSH. Therefore, redox buffers containing extend thiol and its corresponding disulfide were expected to enhance the folding rate of reduced BPTI and reduce the formation mixed disulfide folding intermediates compared to QAS thiol and disulfide.

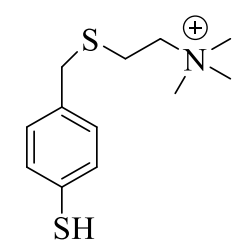

13

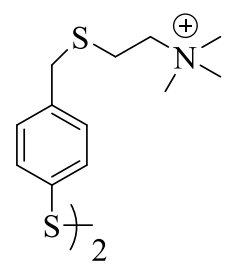

14

Extended thiol

Extended disulfide

Figure 54 Structures of extended thiol and its disulfide

\subsection{Experimental section}

\subsubsection{Extended thiol and disulfide purification}

Extended thiol was synthesized in the lab and the corresponding disulfide was prepared by stirring the thiol solution in air until the thiol was oxidized to the disulfide. Samples were taken from the thiol solution and analyzed by RP-HPLC on a Vydac C18 analytical column until the thiol oxidization was complete. Extended thiol and its disulfide 
were purified by RP-HPLC on a Vydac C18 semi-preparative column. Samples of extended thiol and disulfide were manually injected on to the HPLC and the sample volume was 2 $\mathrm{mL}$ for each injection. Two solvents were used in HPLC purification. Solvent A was $0.1 \%$ TFA in water, and solvent B was $90 \%$ acetonitrile in water with $0.1 \%$ TFA. To elute the sample from the column, a linear gradient was used: 0 min, $90 \%$ solvent A; 4 min, $85 \%$, 20 min, $75 \%$ solvent A; 30 min, $60 \%$ solvent A; 50 min, 55\% solvent $\mathrm{A}$. The absorbance was monitored at $252 \mathrm{~nm}$, and the flow rate was maintained at $2 \mathrm{~mL} / \mathrm{min}$. The purity of all collected sample fractions was determined using a Vydac C18 analytical column. The linear gradient to analyze the sample fractions was 0 min, $90 \%$ solvent $\mathrm{A} ; 40 \mathrm{~min}, 60 \%$ solvent A. All sample fractions containing pure extended thiol or disulfide were combined and lyophilized in the freeze dryer. The lyophilized extended thiol and disulfide samples were dissolved in deoxygenated water respectively and stored at $-20{ }^{\circ} \mathrm{C}$ for oxidative folding studies of reduced BPTI.

\subsection{2 pKa determination of extended thiol}

The extended thiol pKa value was determined using the UV-Vis method. Buffer solutions with varying $\mathrm{pH}$ values ranging from 2.5-11 with a concentration of $50 \mathrm{mM}$ were prepared as described previously, including glycine buffer $(\mathrm{pH} 2.5-2.2$ and $\mathrm{pH}$ 9.0-10.5), 2,2-dimethylsuccinate buffer ( $\mathrm{pH} 3.7-6.7)$, Tris buffer ( $\mathrm{pH}$ 7.0-8.7), and phosphate buffer $(\mathrm{pH}=11)$. Extended thiol solution was diluted with deoxygenated $\mathrm{H}_{2} \mathrm{O}$ to a final concentration of $1 \mathrm{mM}$. Extended thiol solution $(100 \mu \mathrm{L})$ was then mixed with $900 \mu \mathrm{L}$ of each buffer solution. The UV-Vis spectra of all 24 sample solutions was measured on a UV-Vis spectrophotometer. The $\lambda_{\max }$ values of extended thiol and the corresponding thiolate were determined by UV spectra. The absorbance data at the $\lambda_{\max }$ of the thiol were 
plotted as a function of buffer $\mathrm{pH}$. The plot was compared with the plots on the basis of the theory. Then the pKa value of extended thiol was calculated from the best-fit curve.

\subsubsection{Folding of reduced BPTI with extended thiol and disulfide}

A stock solution of reduced BPTI with $1 \mathrm{mg} / \mathrm{mL}$ was diluted with $1.5 \times$ refolding buffer ( $\mathrm{pH} 7.3$ ) to a final concentration of $30 \mu \mathrm{M}$. Folding reactions of reduced BPTI were conducted in a $25{ }^{\circ} \mathrm{C}$ water bath under argon at $\mathrm{pH} 7.3$ in the presence of different concentration combinations of extended thiol and its corresponding disulfides. At specific time points $(5,15,30,60,120$ and $240 \mathrm{~min}), 300 \mu \mathrm{L}$ aliquots of the reaction mixture were removed from the vial and quenched with $80 \mu \mathrm{L}$ of formic acid and stored on an ice bath immediately. Since the peak of extended disulfide overlapped with the peaks of protein folding intermediates on the chromatograms, all acid-quenched folding mixtures were purified on a pipet column with Sephadex G-25 resin as the solid phase to remove small molecule thiol and disulfide. Protein samples were eluted from the column with $0.01 \mathrm{~N}$ $\mathrm{HCl}$ and then analyzed on a Vydac C18 analytical column. The HPLC eluent was monitored at $229 \mathrm{~nm}$ and the column temperature was maintained at $50{ }^{\circ} \mathrm{C}$. A linear gradient with a flow rate of $1 \mathrm{~mL} / \mathrm{min}$ was utilized: 0 min, $90 \%$ solvent $\mathrm{A} ; 40 \mathrm{~min}, 60 \%$ solvent A. The best conditions of extended thiol and its corresponding disulfide in terms of refolding time to obtain $90 \%$ native protein were determined. The rate constants and initial folding rates were calculated by fitting the folding results to a single exponential curve, protein yield $\%=\mathrm{A}\left(1-\mathrm{e}^{-k \mathrm{t}}\right)$, where $\mathrm{A}$ is the maximal folding yield and $k$ is the folding rate constant. 


\subsection{Results and discussion}

\subsubsection{Extended thiol pKa determination}

Extended thiol and its thiolate has distinguishable spectra as the thiol absorbed at 250 $\mathrm{nm}$ and the thiolate absorbed at $275 \mathrm{~nm}$ (Figure 55). The isosbestic point of the extended thiol solution was at $259 \mathrm{~nm}$. The extended thiol $\mathrm{pKa}$ value was determined by comparing the absorbance at $250 \mathrm{~nm}$ against buffer $\mathrm{pH}$ with theoretical values for the distribution of the thiol and thiolate at different $\mathrm{pH}$. The $\mathrm{pKa}$ of extended thiol was calculated to be 6.0, which was higher than that of QAS thiol (Figure 56).

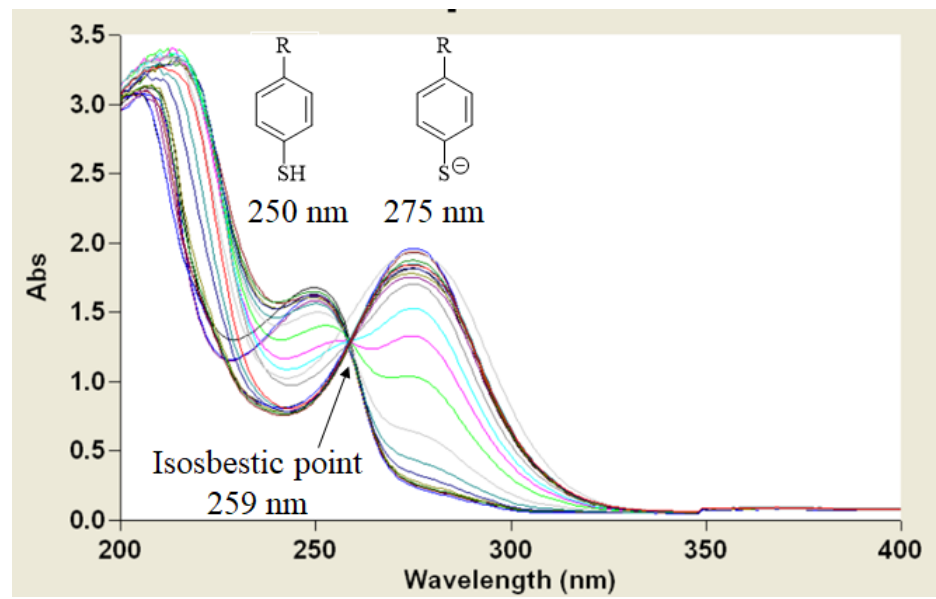

Figure $55 \mathrm{UV}-\mathrm{Vis}$ spectra of extended thiol at various $\mathrm{pH}$

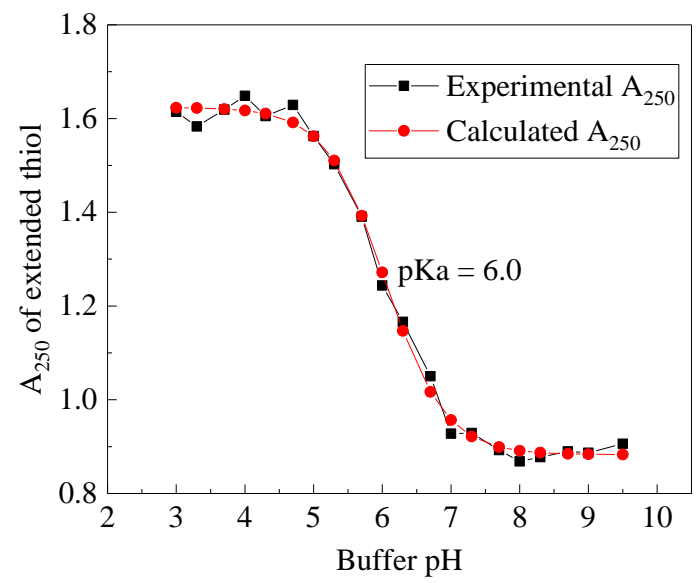

Figure 56 Extended thiol pKa determination 


\subsubsection{Folding of reduced BPTI with extended thiol and disulfide}

Reduced BPTI was folded in the presence of different concentrations of extended disulfide $(0.09,0.25$ and $1 \mathrm{mM})$. Protein precipitation occurred when the folding was conducted with $1 \mathrm{mM}$ extended disulfide leading to low protein peak intensities on the HPLC chromatograms (Figure 57). More than $75 \%$ native protein was obtained with 0.25 $\mathrm{mM}$ disulfide in $8 \mathrm{~h}$, which was higher than the folding yield of $70 \%$ native protein from the folding with $0.09 \mathrm{mM}$ extended disulfide (Figure 58).

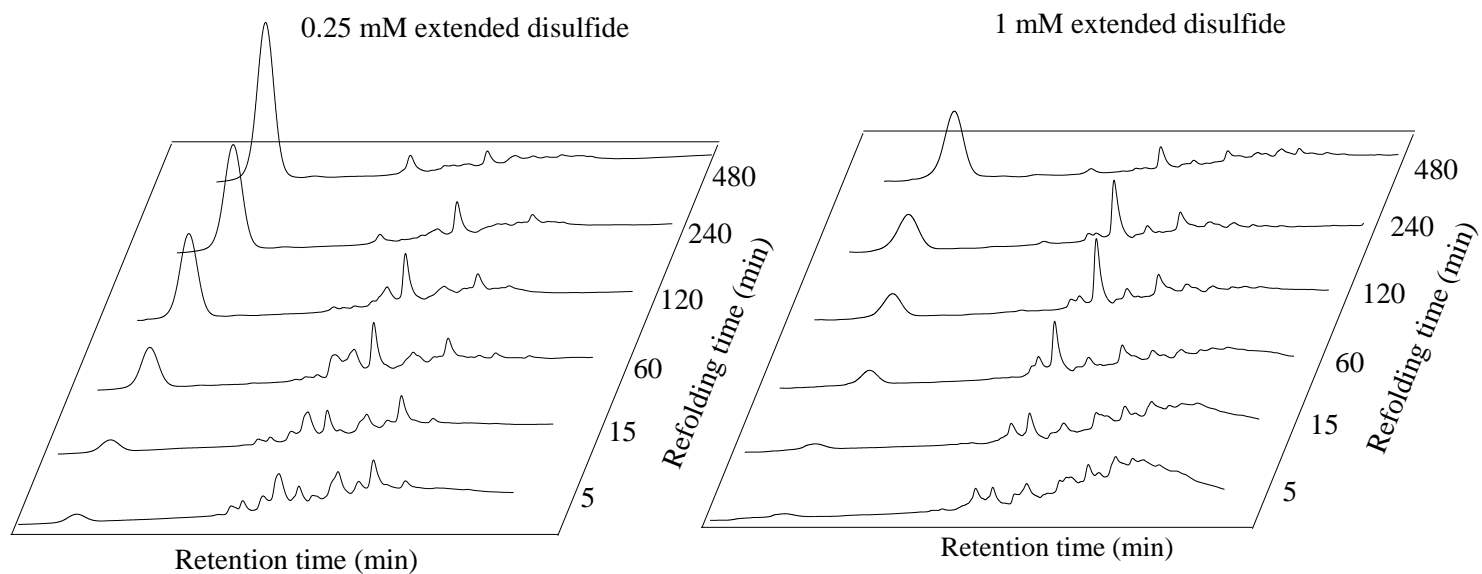

Figure 57 HPLC chromatograms of reduced BPTI folding with extended disulfide

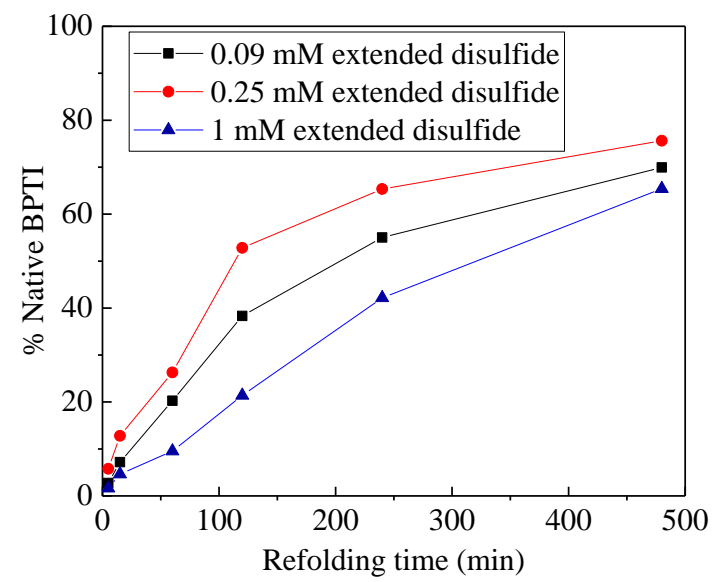

Figure 58 Folding of reduced BPTI with extended disulfide 


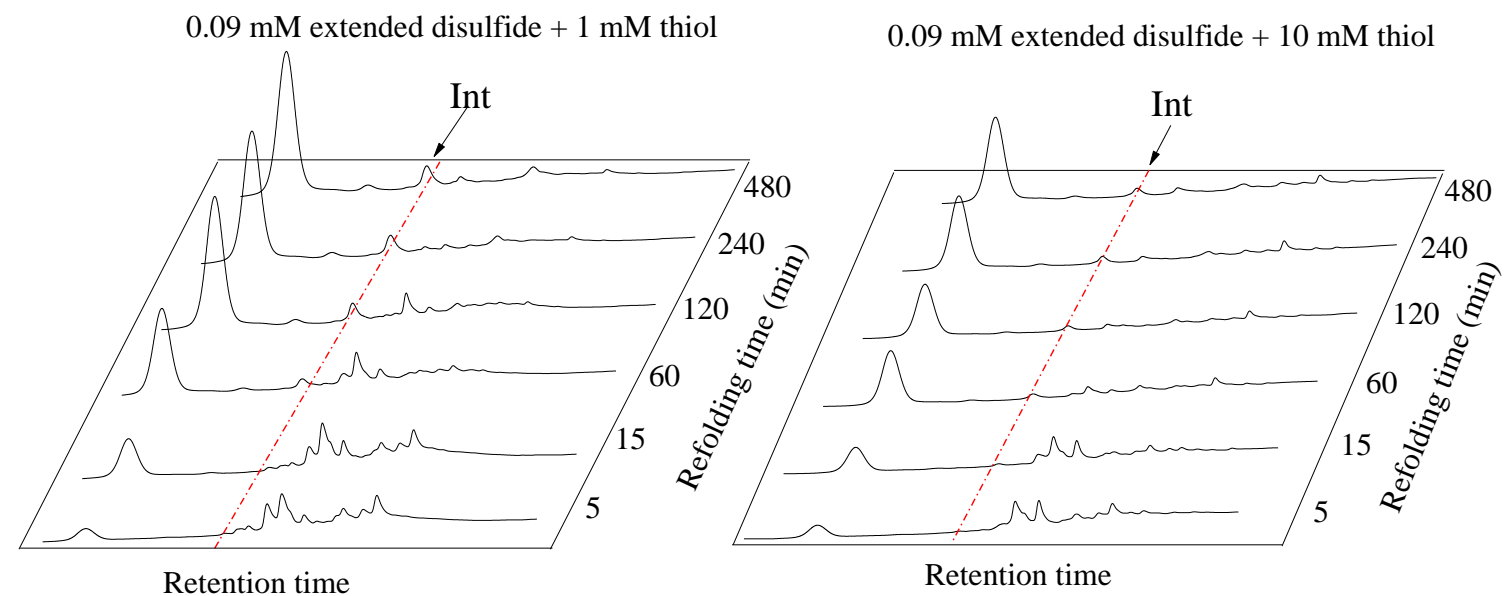

Figure 59 HPLC chromatograms of reduced BPTI folding with $0.09 \mathrm{mM}$ extended disulfide and thiol
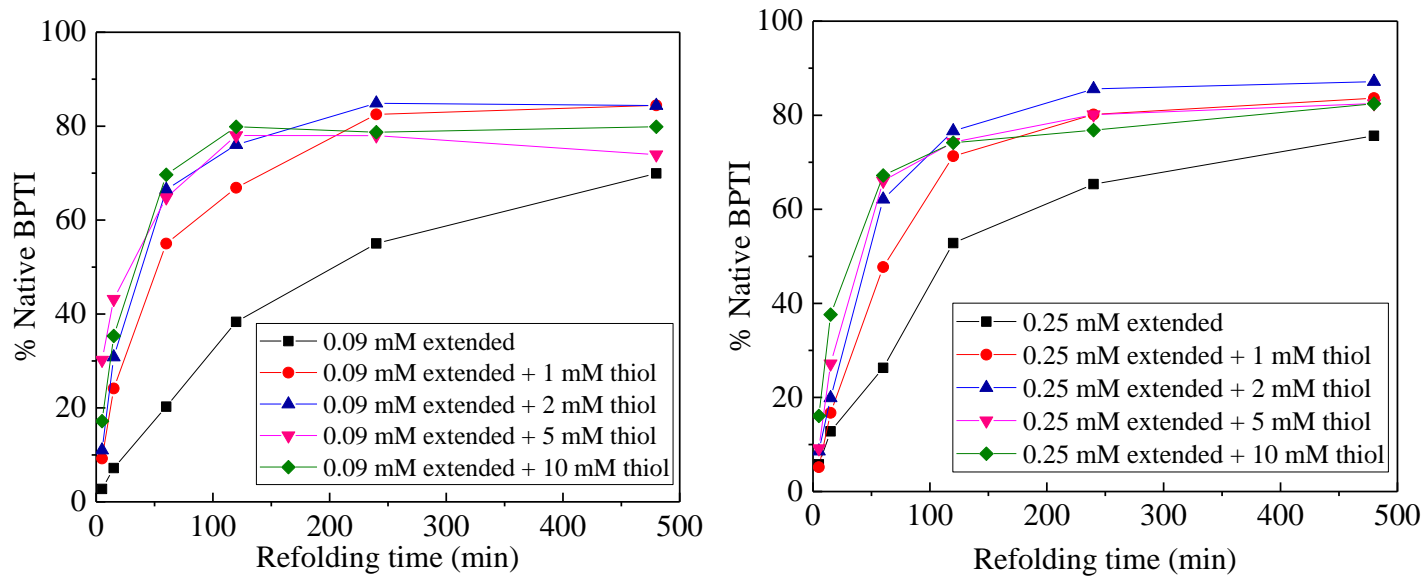

Figure 60 Folding of reduced BPTI with extended disulfide and thiol

Redox buffers containing different concentration combinations of extended disulfide $(0.09$ and $0.25 \mathrm{mM})$ and thiol $(1,2,5$ and $10 \mathrm{mM})$ were used to fold reduced BPTI. Protein precipitation was observed with extended disulfide and high concentrations of thiol (5 and $10 \mathrm{mM}$ ) (Figure 59). After $8 \mathrm{~h}$ folding time, some protein folding intermediates still remained kinetically stable on the HPLC chromatograms, leading to the formation of less than $90 \%$ of native protein (Figure 60). The best redox buffer condition to fold reduced BPTI with extended disulfide and thiol was determined to be $0.25 \mathrm{mM}$ extended disulfide 
and $2 \mathrm{mM}$ thiol, which showed about $87 \%$ of native protein was produced in $8 \mathrm{~h}$ and the initial rate of the reaction was $1.70 \pm 0.10 \% / \mathrm{min}$ (Table 8 ).

Table 8 Folding of reduced BPTI with extended disulifde and thiol

\begin{tabular}{|c|c|c|c|c|}
\hline Redox bu & ffers & & & \\
\hline $\begin{array}{l}\text { Hexyl disulfide } \\
(\mathrm{mM})\end{array}$ & $\begin{array}{l}\text { Hexyl thiol } \\
(\mathrm{mM})\end{array}$ & $\begin{array}{c}\mathrm{A} \\
\text { (\% native protein) }\end{array}$ & $\begin{array}{c}k \\
\left(\min ^{-1}\right)\end{array}$ & $\begin{array}{l}\text { Initial rate } \\
(\% / \mathrm{min})\end{array}$ \\
\hline & 1 & $82 \pm 3$ & $0.018 \pm 0.002$ & $1.48 \pm 0.20$ \\
\hline 009 & 2 & $83 \pm 2$ & $0.028 \pm 0.002$ & $2.31 \pm 0.21$ \\
\hline 0.09 & $5 *$ & $74 \pm 4$ & $0.069 \pm 0.016$ & $5.07 \pm 1.19$ \\
\hline & $10 *$ & $79 \pm 1$ & $0.040 \pm 0.003$ & $3.14 \pm 0.22$ \\
\hline & 1 & $84 \pm 1$ & $0.015 \pm 0.001$ & $1.23 \pm 0.05$ \\
\hline 0.25 & 2 & $87 \pm 1$ & $0.020 \pm 0.001$ & $1.70 \pm 0.10$ \\
\hline & $5 *$ & $80 \pm 1$ & $0.027 \pm 0.002$ & $2.18 \pm 0.15$ \\
\hline & $10 *$ & $77 \pm 2$ & $0.042 \pm 0.005$ & $3.22 \pm 0.41$ \\
\hline
\end{tabular}

*Protein precipitation occurred during folding.

\subsection{Conclusion}

Nucleophilic attack of a thiolate ion on a disulfide is involved in thiol disulfide interchange reactions, so the concentration of the thiolate ion has important effects on the folding reaction rate during the oxidative folding process of disulfide-containing proteins. The active form thiolate concentration is a function of the total thiol concentration in the reaction and the thiol $\mathrm{pKa}$ value. Folding of reduced BPTI using aromatic thiols with lower pKa values will have higher folding reaction rate at the same folding conditions, but tend to form more mixed disulfides between the protein and the aromatic thiol because of high reactivity of the corresponding aromatic disulfide. Compared to aromatic thiol QAS with a pKa value of 5.5, the newly synthesized extended thiol had a higher $\mathrm{pKa}$ value which was determined to be 6.0. The folding of reduced BPTI with extended thiol and disulfide was expected to be slower than the folding with QAS thiol and disulfide, but with fewer formation of mixed disulfide intermediates. However, when extended thiol and its 
corresponding disulfide were used in the redox buffers to fold reduced BPTI, less than $90 \%$ of native protein was produced in $8 \mathrm{~h}$. When folding of reduced BPTI with higher concentrations of extended thiol and disulfide, protein precipitation was observed. More aromatic thiols with different $\mathrm{pKa}$ values should be used to fold reduced BPTI at same folding conditions in order to better determine the relationship between thiol $\mathrm{pKa}$ values and the folding reaction rates. 


\section{Folding analysis of reduced BPTI using capillary electrophoresis}

\subsection{Abstract}

A new method to analyze the folding of reduced BPTI with QAS disulfide was developed using capillary electrophoresis (CE) on an uncoated silica capillary. Phosphate buffers with a low $\mathrm{pH}$ value $(\mathrm{pH}=2.5)$ were utilized to reduce the deprotonation of silanols on the inner surface of the capillary. The addition of hydroxyethyl cellulose (HEC) to the buffers minimized the adsorption of positively charged proteins to the silica capillary wall. The capillary was also flushed with $0.1 \mathrm{M} \mathrm{HCl}$ to maintain the silanol groups on the capillary wall fully protonated and suppress the EOF. Folding reaction mixtures of reduced BPTI were analyzed within 20 min on CE. A more rapid and sensitive method to analyze the oxidative folding process of reduced BPTI on $\mathrm{CE}$ was obtained relative to traditional HPLC analysis.

\subsection{Introduction}

Since the early 1980s, Capillary electrophoresis (CE) has become a powerful analytical technique for many macromolecules including proteins and DNA fragments. Compared to traditional HPLC analysis, CE has many advantages, such as minute amounts of sample required, short analysis time, high resolution and efficiency. In CE methods, analytes can migrate with different velocities through electrolyte solutions on the basis of their different properties such as charge, mass or the partitioning between two different phases. Therefore, different types of CE methods have been developed, including capillary zone electrophoresis (CZE), capillary isoelectric focusing (CIEF) and micellar electrokinetic chromatography (MEKC) ${ }^{87}$ Among all $\mathrm{CE}$ techniques, CZE is the most commonly used and simplest method. The separation by CZE is based on the 
electrophoretic mobility differences of analytes in an electric field ${ }^{88}$ Electrophoretic mobility is related to the size, friction and charge of each analyte. Peaks for analytes with different electrophoretic mobility appear on the electropherogram when analytes pass through UV-Vis detector at specific wavelengths.

Protein adsorption can occur through ionic and nonionic interactions between the silica wall of the capillary and the protein, especially when the separation is conducted at the $\mathrm{pH}$ value below the isoelectric point $(\mathrm{pI})$ of the protein. ${ }^{89}$ Peak broadening and tailing are likely to be observed during the separation of proteins on an uncoated silica capillary, leading to low separation efficiencies. Since the pKa of $\mathrm{SiOH}$ is about 3 , the inner wall of the uncoated silica capillary is negatively charged because of the deprotonation of $\mathrm{SiOH}$ to $\mathrm{SiO}^{-}$when the buffer $\mathrm{pH}$ is above 3, resulting in a bulk electroosmotic flow (EOF) in the capillary under the applied electric filed. ${ }^{90}$ Approaches to minimize protein adsorption in CE analysis have been demonstrated in many studies..$^{91-92}$ The simplest way is to use separation buffers with extreme $\mathrm{pH}$ values to keep the silanol groups fully protonated or ionized. With low buffer $\mathrm{pH}$, silanol groups on the capillary wall tend to be protonated, thereby reducing the electrostatic interactions with protein analytes and the magnitude of the EOF concomitantly. In addition, proteins are positively charged at $\mathrm{pH}$ lower than the $\mathrm{pI}$ values, which assures that all proteins can migrate toward the same direction in the electric field. ${ }^{93}$ Capillary wall coatings, including dynamic or static coatings, are used to reduce protein-wall interactions and alter the EOF. Small molecule additives such as amines and zwitterionic reagents can significantly reduce protein adsorption by interacting with the negatively charged silanol groups on the capillary wall. The addition of protonated positively charged amines to the background electrolyte can slow down the EOF and 
improve protein separation efficiencies. ${ }^{94}$ High concentrations of zwitterions can minimize protein adsorption to the silica wall and prevent protein-protein interactions during $\mathrm{CE}$ analysis. Since zwitterionic reagents do not contribute to the ionic strength and conductivity of the buffer solutions, high separation voltage can be applied to the $\mathrm{CE}$ process to shorten the migration time of analytes. ${ }^{95-96}$ Polymer wall coatings of silica capillary have also been widely used by adding water soluble polymers to the buffer solutions. Capillaries dynamically coated with polymers, such as polyethylene oxide, cellulose and their derivatives, showed decreased EOF under basic $\mathrm{pH}$ buffer conditions. At lower $\mathrm{pH}$, higher protein separation efficiencies were obtained using polymer coated capillaries, especially for basic proteins. ${ }^{97}$

Since the pI value of BPTI is close to 10.5, it has positive net charges when the buffer $\mathrm{pH}$ is lower than its $\mathrm{pI}$ value. In the present study, the folding process of reduced BPTI with QAS disulfide was analyzed using CE-UV. Native protein and mixed disulfide folding intermediates with different molecular weight and charges were expected to be separated under an applied electric field in CE. Phosphate buffers with $\mathrm{pH} 2.5$ were used in the process which reduced the negative charges on the inner surface of the capillary. Hydroxyethyl cellulose (HEC) was used as a BGE additive in order to suppress protein adsorption to the silica capillary wall and improve protein separation efficiencies. The sensitivity of CE is limited when the sample has a low concentration and only a minute amount of sample is injected into capillary for analysis compared to HPLC analysis. Therefore, sample stacking was conducted during the $\mathrm{CE}$ analysis in order to achieve better sensitivity. Protein samples were prepared in $1 \mathrm{mM} \mathrm{HCl}$ in order to have lower ionic strength than the running buffer ( $0.02 \mathrm{M}$ phosphate buffer) for $\mathrm{CE}$ analysis. Hydrodynamic 
injection with a long injection time (300 s) was used to introduce large sample volumes to the capillary. The difference in conductivity caused that the analytes migrated rapidly and concentrated at the boundary between the sample solution and the running buffer.

\subsection{Experimental section}

\subsubsection{Materials}

Sodium phosphate monobasic monohydrate $\left(\mathrm{NaH}_{2} \mathrm{PO}_{4} \cdot \mathrm{H}_{2} \mathrm{O}\right)$ for electrophoresis was purchased from Sigma Aldrich. Phosphoric acid $\left(\mathrm{H}_{3} \mathrm{PO}_{4}\right)$, sodium hydroxide $(\mathrm{NaOH})$ and 2-hydroxyethyl cellulose (HEC, MW = 90,000) were purchased from Fisher Scientific. $\epsilon$ Amino-N-caproic acid (EACA) was purchased from MP Biomedicals.

All experiments were performed on an Agilent Technologies equipped with a diode array detector. Data acquisition was performed with Chemstations software. The detection was monitored by UV at 214 and $280 \mathrm{~nm}$. The capillary temperature was $25{ }^{\circ} \mathrm{C}$ for all the experiments. Electrophoretic analysis was performed on an uncoated fused-silica capillary (total length of $48 \mathrm{~cm}$, effective length of $40 \mathrm{~cm}$, i.d. of $75 \mu \mathrm{m}$, o.d. of $363 \mu \mathrm{m}$ ) from Polymicro Technologies. Prior to the first use, the capillary was conditioned with 0.1 $\mathrm{M} \mathrm{NaOH}$ and deionized water for $15 \mathrm{~min}$, respectively. Before daily use, the capillary was conditioned with $0.1 \mathrm{M} \mathrm{HCl}$, deionized water, and BGE (running buffer) for $5 \mathrm{~min}$, consecutively.

\subsubsection{Protein separation using CE-UV}

A stock solution of native BPII was prepared by dissolving the protein with $1 \mathrm{mM} \mathrm{HCl}$ to a final concentration of $0.1 \mathrm{mg} / \mathrm{mL}$. A series of native BPTI solutions with varying concentrations was prepared from the stock solution. Reduced BPTI was prepared and

purified as described in chapter 3. Lyophilized reduced BPTI was diluted to $0.1 \mathrm{mg} / \mathrm{mL}$ 
with 1mM HCl. All protein samples were analyzed using CE-UV. Phosphate buffers ( $\mathrm{pH}$ 2.5) were used in CE-UV analysis. The rinsing buffer was $0.04 \mathrm{M}$ phosphate buffer ( $\mathrm{pH}$ 2.5) prepared using $\mathrm{NaH}_{2} \mathrm{PO}_{4}$ and $\mathrm{H}_{3} \mathrm{PO}_{4}$. The running buffer was $0.02 \mathrm{M}$ phosphate buffer $(\mathrm{pH}$ 2.5). Prior to each injection, the capillary was preconditioned with the rinsing buffer for $1 \mathrm{~min}$ and the running buffer for $3 \mathrm{~min}$. Protein samples were injected hydrodynamically to the capillary at $5 \mathrm{mbar}$ for $300 \mathrm{~s}$. The applied separation voltage was $20 \mathrm{kV}$, and the absorbance was monitored by UV at 214 and $280 \mathrm{~nm}$. After each injection, the capillary was postconditioned with deionized water for 2 min. In order to minimize protein adsorption on the uncoated silica capillary wall, different concentrations of HEC were added to the rinsing and running buffers for $\mathrm{CE}$ analysis.

\subsubsection{Protein folding analysis of reduced BPTI using CE-UV}

Reduced BPTI $(30 \mu \mathrm{M})$ was folded with $0.25 \mathrm{mM}$ QAS disulfide in the folding buffer containing $0.10 \mathrm{M}$ bis-tris propane, $0.20 \mathrm{M} \mathrm{KCl}$ and $1.0 \mathrm{mM}$ EDTA at $\mathrm{pH} 7.3$ in a $25^{\circ} \mathrm{C}$ water bath. At certain folding time points, $300 \mu \mathrm{L}$ aliquots of the folding reaction mixture were removed and quenched with $80 \mu \mathrm{L}$ formic acid. Each acid-quenched reaction sample was loaded to a Sephadex G-25 pipet column. Protein sample fractions were eluted using $1 \mathrm{mM} \mathrm{HCl}$ as the mobile phase. Sample fractions containing protein were collected and analyzed by CE-UV. The CE separation for protein folding analysis was conducted as described above.

\subsection{Results and discussion}

\subsubsection{Protein separations using phosphate buffers (pH 2.5)}

A separation of native and reduced BPTI was conducted with capillary electrophoresis on an uncoated silica capillary (Figure 61). A phosphate buffer (0.02 M, pH 2.5) was used 
as the running buffer to suppress the charge effects on the silica capillary wall. Native and reduced BPTI were separated within 20 min. However, broad peaks and low intensities were obtained for both proteins on the electropherograms, indicating the occurrence of protein absorption to the inner surface of the capillary wall.

The uncoated capillary was conditioned with $0.1 \mathrm{M} \mathrm{HCl}$ before runs to diminish the EOF and remove the adsorbed proteins from the inner wall of the capillary. Acid flushing also kept the silanol groups on the capillary wall protonated. Native BPTI was diluted with $1 \mathrm{mM} \mathrm{HCl}$ to different concentrations $(0.001-0.1 \mathrm{mg} / \mathrm{mL})$ and analyzed with CE (Figure 62). The protein analysis was reproducible and the migration time $\%$ RSD was about $2.22 \%$. After acid flushing of the capillary, protein peak width was reduced and high absorbance intensity of native BPTI was observed on the electropherogram indicating the reduced protein adsorption on the silica wall of the capillary. Good linearity for the absorbance of native BPTI at $214 \mathrm{~nm}$ against the protein sample concentration was obtained with a high correlation coefficient $\left(\mathrm{R}^{2}\right)$ of 0.9946 (Figure 63).

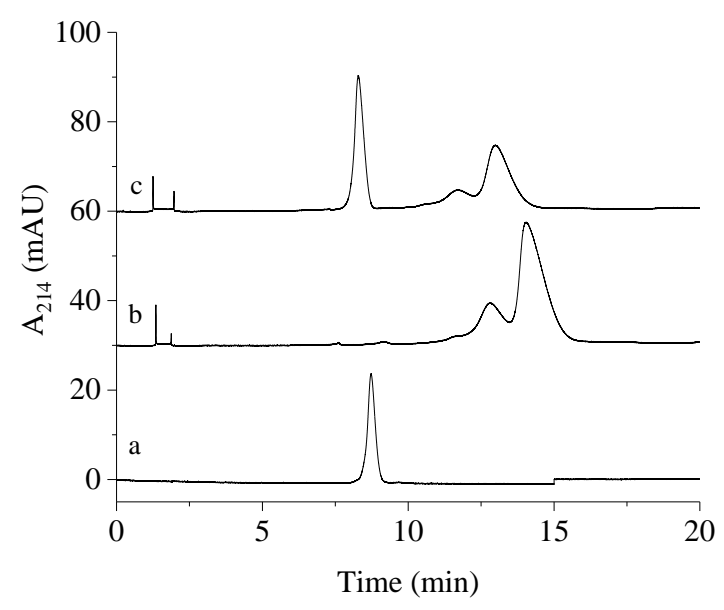

Figure 61 Electropherograms of native and reduced BPTI a: Native BPTI; b: Reduced BPTI; c: Native and reduced BPTI 


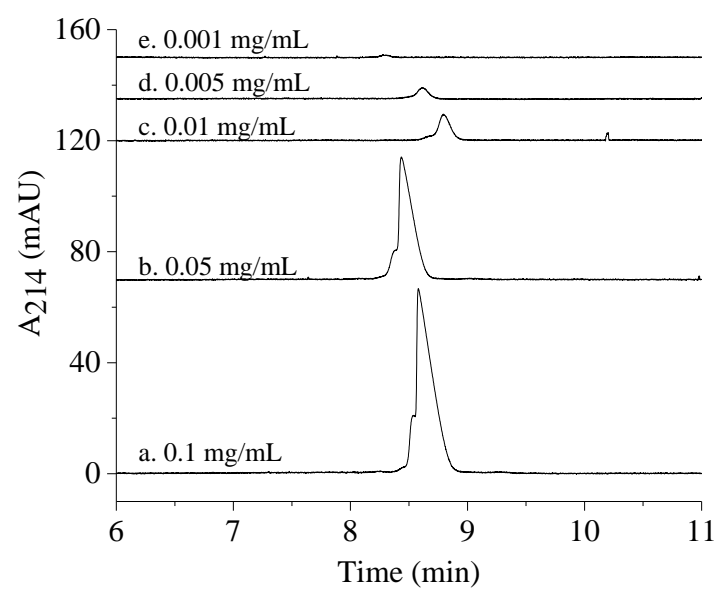

Figure 62 Electropherograms of native BPTI using phosphate buffers

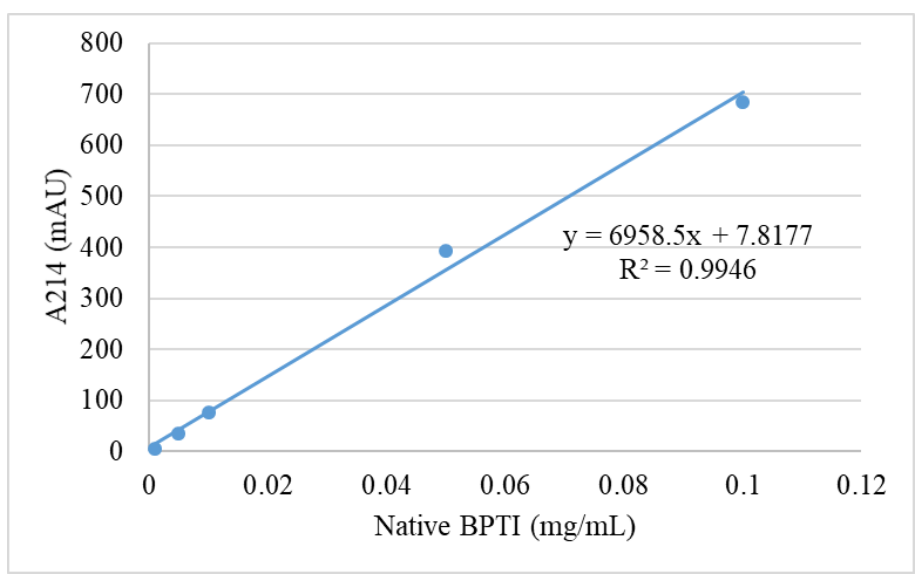

Figure $63 \mathrm{CE}$ analysis of native BPTI using phosphate buffers

Reduced BPTI was folded with $0.25 \mathrm{mM}$ QAS disulfide at $\mathrm{pH} 7.3$, and $300 \mu \mathrm{L}$ of the folding reaction mixture were quenched with formic acid at each folding time point $(5,15$, 60, 120 and $480 \mathrm{~min}$ ). Acid-quenched reaction mixtures were loaded to a G-25 pipet column and eluted with $1 \mathrm{mM} \mathrm{HCl}$ to remove small molecules. The eluted protein samples were analyzed by CE using $0.02 \mathrm{M}$ phosphate buffer $\mathrm{pH} 2.5$ as the running buffer. Only two major peaks were observed from the electropherogram. According to HPLC folding analysis, more protein intermediates were obtained at the same folding condition, 
indicating that protein samples were not well separated on CE using current buffer condition.

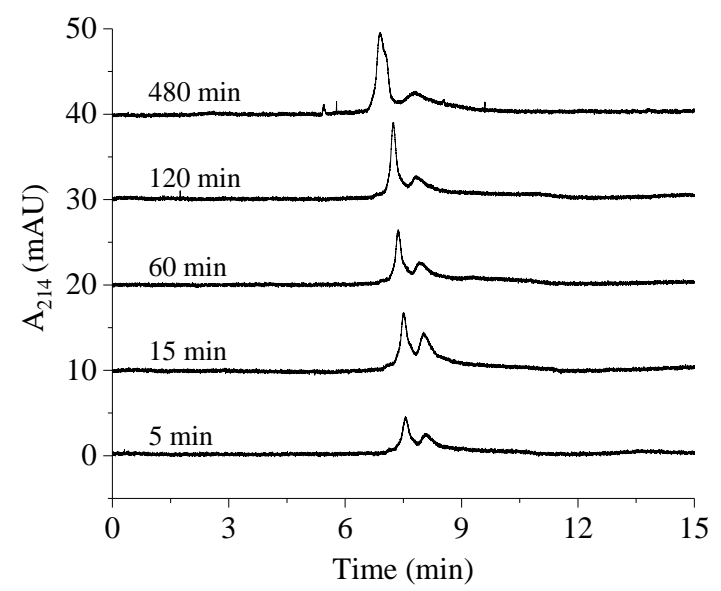

Figure 64 Electropherograms of reduced BPTI folding with $0.25 \mathrm{mM}$ QAS disulfide

\subsubsection{Protein separations using phosphate buffers (pH 2.5) with additives}

\subsubsection{The addition of hydroxyethyl cellulose (HEC)}

In order to minimize the adsorption of protein to the inner wall of the capillary, hydroxyethyl cellulose (HEC) was added to the running buffer. Compared to the use of $0.02 \mathrm{mM}$ phosphate buffer ( $\mathrm{pH} 2.5$ ) as the running buffer, the suppression of EOF during protein analysis was observed when adding $0.1 \%$ HEC to the buffer since the migration time of native BPTI was prolonged to 14 min with \% RSD of $4.80 \%$ (Figure 65). A higher intensity of the native protein peak at $214 \mathrm{~nm}$ was observed. The linear fit of the absorbance of native protein against protein concentration was obtained with a correlation coefficient $\left(\mathrm{R}^{2}\right)$ of 0.9993 (Figure 66). When the concentration of HEC in the buffer was increased to $0.2 \%$, lower protein absorbance was obtained and the efficacy of HEC in preventing protein adsorption was not increased. Similar observations were shown in previous studies, 
indicating that an intermediate HEC level in the buffer solution tended to inhibit protein adsorption to the silica capillary wall. ${ }^{98}$

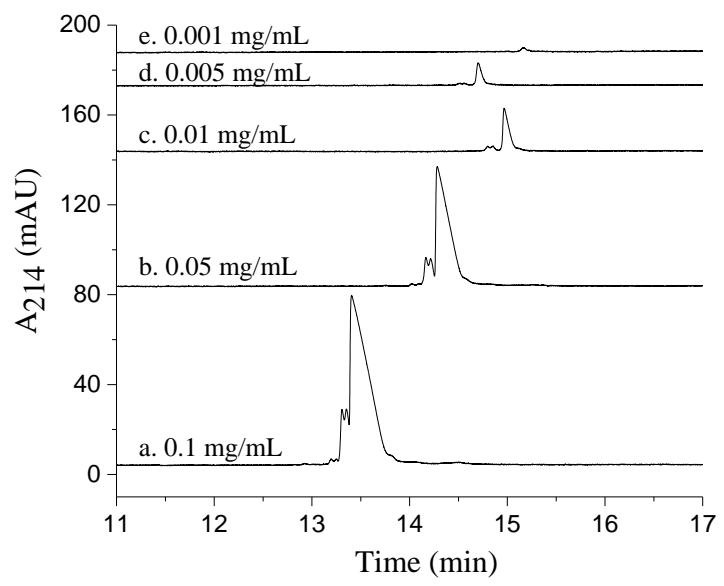

Figure 65 Electropherograms of native BPTI using phosphate buffers with $0.1 \%$ HEC

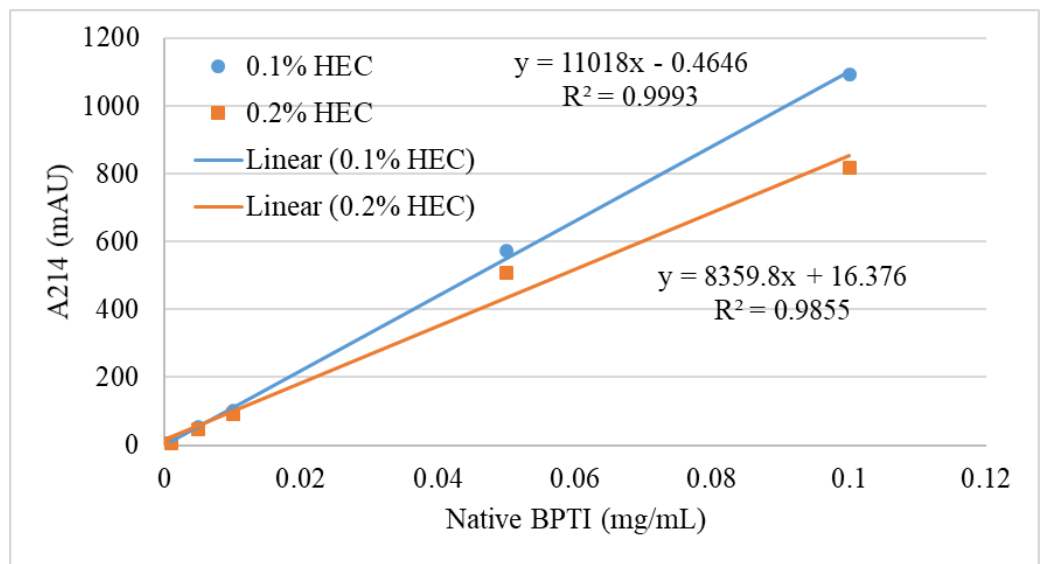

Figure 66 Native BPTI analysis using phosphate buffers with HEC

Protein folding sample fractions were next analyzed by CE. Using phosphate buffer $\mathrm{pH} 2.5$ and $0.1 \%$ HEC as the running buffer, protein separation was obtained in $20 \mathrm{~min}$. Protein folding intermediate peaks were shown on the CE electropherogram with better resolution. More protein intermediate peaks were observed within a shorter analysis time (Figure 67). The yield of native protein was calculated using the ratio of native protein 
peak versus total peak area. Protein folding intermediates converted to native protein when increasing the refolding time, resulting in the increase of native protein yield (Figure 68).

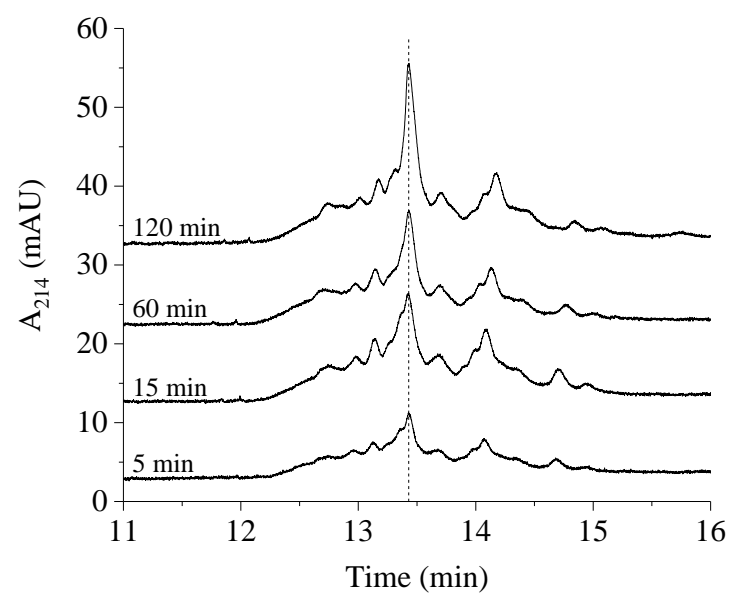

Figure 67 Electropherograms of reduced BPTI folding with $0.25 \mathrm{mM}$ QAS disulfide using phosphate buffers with $0.1 \%$ HEC

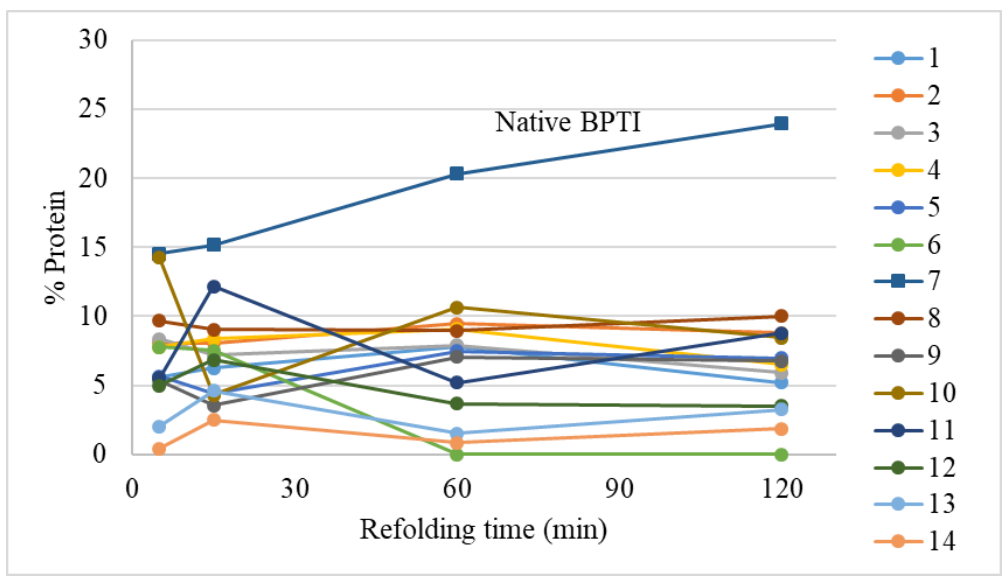

Figure 68 Folding analysis of reduced BPTI with $0.25 \mathrm{mM}$ QAS disulfide

\subsubsection{The addition of $\epsilon$-amino-N-caproic acid (EACA)}

Previous studies have shown that the addition of high concentrations (up to $600 \mathrm{mM}$ ) of a zwitterionic agent EACA in the running buffer to effectively reduces the interaction of protein analytes with the inner wall of the bare silica capillary without increasing joule heating in CE analysis. ${ }^{99-100}$ The background electrolyte with low UV absorption allowed 
the detection of protein analysis at $214 \mathrm{~nm}$ with high sensitivity. However, when $600 \mathrm{mM}$ EACA-phosphate buffer ( $\mathrm{pH}$ 5.5) was used as the running buffer to analyze native BPTI on CE-UV, an asymmetrical peak with low intensity was observed on the electropherogram. The sensitivity of protein analysis on CE was not increased by the addition of EACA in the running buffer, possibly due to the use of long sample stacking injection method and low protein sample concentration.

\subsection{Conclusion}

A method using CE-UV has been developed for the oxidative folding analysis of reduced BPTI with small molecule QAS disulfide. The method allowed rapid separation of reduced and native BPTI with good reproducibility and high sensitivity. After the folding of reduced BPTI with $0.25 \mathrm{mM}$ QAS disulfide, protein folding intermediates with different sizes and charges were expected to be separated on CE. Capillary conditioning with $0.1 \mathrm{M} \mathrm{HCl}$ was shown to be an effective way to maintain reproducibility of protein separations from injection to injection. Phosphate buffers containing $0.1 \%$ HEC ( $\mathrm{pH} 2.5$ ) were utilized in order to reduce protein adsorption to the silica capillary wall during the protein separation analysis on CE. Compared to traditional HPLC analysis, CE-UV provided a new method to analyze the folding process of reduced BPTI with low solvent consumption and shorter analysis time. 


\section{Conclusions and future research}

Reduced BPTI was folded with different aromatic thiols and their corresponding disulfides in vitro. The best folding conditions and reaction rate constants were determined by changing the concentration combinations of each aromatic thiol and its disulfide.

Three aromatic thiols, including negatively charged PA and SA thiols and positively charged QAS thiol, showed different efficiencies on the folding of reduced BPTI. At the folding $\mathrm{pH}$ 7.3, BPTI is positively charged as it has a pI value of 10.5. Mixed disulfide folding intermediates were formed during the folding of reduced BPTI with all three thiols and disulfides. Protein precipitation was observed during the process when reduced BPTI was folded with negatively charged PA or SA thiol, presumably due to the minimized net charge of mixed disulfide folding intermediates. In comparison, no protein precipitation occurred during the folding of reduced BPTI with positively charged QAS thiol and disulfide. With the redox buffer containing $0.25 \mathrm{mM}$ QAS disulfide and $10 \mathrm{mM}$ QAS thiol, reduced BPTI was folded to $90 \%$ native protein in $2 \mathrm{~h}$ with an initial rate of $4.90 \pm$ $0.23 \% / \mathrm{min}$, which was determined to be the best folding concentration combination.

The hydrophobicity of small molecule thiols and disulfides was shown to have effects on the folding rate and yield of reduced BPTI. Three aromatic thiols, butyl, hexyl and octyl thiol, were synthesized to have an elongated alkyl group to increase the hydrophobicity of these small molecules compared to QAS thiol with a methyl group on the same position of the structure. Aromatic thiols and disulfides with greater hydrophobicity will increase the interactions with the hydrophobic core of the protein, and should help the formation and rearrangement of mixed disulfide intermediates during folding. However, the results showed that the longer the hydrocarbon chain in the small molecule structure, the higher 
the tendency of protein precipitation. Folding of reduced BPTI with octyl thiol and disulfide formed protein precipitation in all the reaction mixtures. Folding of reduced BPTI with the best concentration combination of hexyl thiol and its disulfide was completed in $1 \mathrm{~h}$ in terms of the formation of $90 \%$ native protein. The initial folding rate of the reduced BPTI folding reaction with $0.25 \mathrm{mM}$ hexyl disulfide and $10 \mathrm{mM}$ hexyl thiol was determined to be $9.42 \pm 0.54 \% / \mathrm{min}$, which was 40 times that of folding with the traditional redox buffer containing $5 \mathrm{mM}$ GSSG and $5 \mathrm{mM}$ GSH.

Folding intermediates formed during the folding of reduced BPTI with the best concentration combinations of aromatic thiols and their corresponding disulfides are expected to be isolated in order to further understand the oxidative folding process. Kinetically stable folding intermediates will be reacted with aromatic thiols and disulfides respectively and the folding rate constants and initial folding rates will be determined. With further characterization of the folding intermediates, the oxidative folding mechanism of reduced BPTI with aromatic thiols and disulfides will be elucidated. The rate constants of the folding intermediates will be used to model the oxidative folding pathways of reduced BPTI using aromatic thiols and their corresponding disulfides.

A new method to analyze the folding of reduced BPTI with small molecule aromatic disulfide was developed using CE on an uncoated silica capillary. The analysis was conducted at $\mathrm{pH} 2.5$ to reduce deprotonation of silanol groups on the capillary wall. The separation of native and reduced BPTI was completed in a short run time with injection of only a small amount of sample. Good linearity with high correlation coefficient was obtained for the analysis of native protein with the concentration ranging from $0.001-0.1$ $\mathrm{mg} / \mathrm{mL}$. The folding of reduced BPTI with $0.25 \mathrm{mM}$ QAS disulfide was analyzed using 
CE, showing that folding intermediates were accumulated at each folding time point and separated on the electropherogram. Dynamic wall coating of the capillary and acid flushing was performed in order to reduce protein adsorption to the silica capillary wall. The addition of HEC to the running buffer effectively reduced protein adsorption and increased protein separation resolution. More capillary wall coating methods, such as the addition of amines and zwitterionic reagents, are expected to be used in protein folding analysis on $\mathrm{CE}$ in order to improve the separation sensitivity and resolution. 


\section{References}

1. Berg, J.; Tymoczko, J.; Stryer, L., Biochemistry. 6th ed.; WH Freeman, New York: 2007.

2. Anfinsen, C. B., Principles that govern the folding of protein chains. Science 1973, 181 (4096), 223.

3. Dill, K. A.; Chan, H. S., From Levinthal to pathways to funnels. Nature Structural Biology 1997, 4, 10.

4. Dobson, C. M., Protein folding and misfolding. Nature 2003, 426 (6968), 884-890.

5. Frand, A. R.; Cuozzo, J. W.; Kaiser, C. A., Pathways for protein disulphide bond formation. Trends in cell biology 2000, 10 (5), 203-10.

6. Lagassé, H. A. D.; Alexaki, A.; Simhadri, V. L.; Katagiri, N. H.; Jankowski, W.; Sauna, Z. E.; Kimchi-Sarfaty, C., Recent advances in therapeutic protein drug development. F1000 Research 2017, 6, 113.

7. Huang, C., Jr.; Lin, H.; Yang, X., Industrial production of recombinant therapeutics in Escherichia coli and its recent advancements. J Ind Microbiol Biotechnol 2012, 39 (3), 383-399.

8. Rosano, G. L.; Ceccarelli, E. A., Recombinant protein expression in Escherichia coli: advances and challenges. Front. Microbiol. 2014, 5, 172.

9. Kamionka, M., Engineering of therapeutic proteins production in Escherichia coli. Current Pharmaceutical Biotechnology 2011, 12 (2), 268-274.

10. Yamaguchi, H.; Miyazaki, M., Refolding techniques for recovering biologically active recombinant proteins from inclusion bodies. Biomolecules 2014, 4 (1), 23551.

11. Hartl, F. U.; Hayer-Hartl, M., Converging concepts of protein folding in vitro and in vivo. Nature Structural \&Amp; Molecular Biology 2009, 16, 574.

12. Soto, C., Protein misfolding and disease; protein refolding and therapy. FEBS Letters 2001, 498 (2-3), 204-207.

13. Ashraf, G. M.; Greig, N. H.; Khan, T. A.; Hassan, I.; Tabrez, S.; Shakil, S.; Sheikh, I. A.; Zaidi, S. K.; Akram, M.; Jabir, N. R.; Firoz, C. K.; Naeem, A.; Alhazza, I. M.; Damanhouri, G. A.; Kamal, M. A., Protein misfolding and aggregation in Alzheimer's disease and type 2 diabetes mellitus. CNS Neurol. Disord. Drug Targets 2014, 13 (7), 1280-93. 
14. Mossuto, M. E.; Bolognesi, B.; Guixer, B.; Dhulesia, A.; Agostini, F.; Kumita, J. R.; Tartaglia, G. G.; Dumoulin, M.; Dobson, C. M.; Salvatella, X., Disulfide Bonds Reduce the toxicity of the amyloid fibrils formed by an extracellular protein. Angew. Chem. Int. Ed. 2011, 50 (31), 7048-7051.

15. Mossuto, M. F., Disulfide bonding in neurodegenerative misfolding diseases. Int. J. Cell Biol. 2013, 2013, 318319.

16. Ágoston, V.; Cemazar, M.; Kaján, L.; Pongor, S., Graph-representation of oxidative folding pathways. BMC Bioinformatics 2005, 6 (1), 19.

17. Pace, C. N.; Grimsley, G. R.; Thomson, J. A.; Barnett, B. J., Conformational stability and activity of ribonuclease T1 with zero, one, and two intact disulfide bonds. The Journal of Biological Chemistry 1988, 263 (24), 11820-5.

18. Wang, L.; Zhang, L.; Niu, Y.; Sitia, R.; Wang, C. C., Glutathione peroxidase 7 utilizes hydrogen peroxide generated by Erolalpha to promote oxidative protein folding. Antioxidants \& Redox Signaling 2014, 20 (4), 545-56.

19. Gilbert, H. F., Thiol/disulfide exchange equilibria and disulfide bond stability. Methods in Enzymology 1995, 251, 8-28.

20. Bach, R. D.; Dmitrenko, O.; Thorpe, C., Mechanism of thiolate-disulfide interchange reactions in biochemistry. The Journal of Organic Chemistry 2008, 73 (1), 12-21.

21. Hudson, D. A.; Gannon, S. A.; Thorpe, C., Oxidative protein folding: from thioldisulfide exchange reactions to the redox poise of the endoplasmic reticulum. Free Radical Biology \& Medicine 2015, 80, 171-82.

22. Wedemeyer, W. J.; Welker, E.; Narayan, M.; Scheraga, H. A., Disulfide bonds and protein folding. Biochemistry 2000, 39 (15), 4207-4216.

23. Narayan, M.; Welker, E.; Wedemeyer, W. J.; Scheraga, H. A., Oxidative folding of proteins. Accounts of Chemical Research 2000, 33 (11), 805-812.

24. Hatahet, F.; Boyd, D.; Beckwith, J., Disulfide bond formation in prokaryotes: history, diversity and design. Biochimica et biophysica acta 2014, 1844 (8), 14021414.

25. Tu, B. P.; Weissman, J. S., Oxidative protein folding in eukaryotes: mechanisms and consequences. J Cell Biol 2004, 164 (3), 341-6.

26. Bardwell, J. C.; McGovern, K.; Beckwith, J., Identification of a protein required for disulfide bond formation in vivo. Cell 1991, 67 (3), 581-9. 
27. Shouldice, S. R.; Cho, S. H.; Boyd, D.; Heras, B.; Eser, M.; Beckwith, J.; Riggs, P.; Martin, J. L.; Berkmen, M., In vivo oxidative protein folding can be facilitated by oxidation-reduction cycling. Mol. Microbiol 2010, 75 (1), 13-28.

28. Depuydt, M.; Messens, J.; Collet, J. F., How proteins form disulfide bonds. Antioxidants \& Redox Signaling 2011, 15 (1), 49-66.

29. Wilkinson, B.; Gilbert, H. F., Protein disulfide isomerase. Biochimica et Biophysica Acta (BBA) - Proteins and Proteomics 2004, 1699 (1-2), 35-44.

30. Bulleid, N. J.; Ellgaard, L., Multiple ways to make disulfides. Trends in Biochemical Sciences 2011, 36 (9), 485-492.

31. Karala, A. R.; Lappi, A. K.; Ruddock, L. W., Modulation of an active-site cysteine pKa allows PDI to act as a catalyst of both disulfide bond formation and isomerization. Journal of Molecular Biology 2010, 396 (4), 883-92.

32. Oka, O. B.; Bulleid, N. J., Forming disulfides in the endoplasmic reticulum. Biochim. Biophys. Acta. 2013, 1833 (11), 2425-9.

33. Gross, E.; Kastner, D. B.; Kaiser, C. A.; Fass, D., Structure of Ero1p, source of disulfide bonds for oxidative protein folding in the cell. Cell 2004, 117 (5), 601610.

34. Tu, B. P.; Weissman, J. S., Oxidative protein folding in eukaryotes: mechanisms and consequences. The Journal of Cell Biology 2004, 164 (3), 341-346.

35. Hwang, C.; Sinskey, A.; Lodish, H., Oxidized redox state of glutathione in the endoplasmic reticulum. Science 1992, 257 (5076), 1496-1502.

36. Tu, B. P.; Ho-Schleyer, S. C.; Travers, K. J.; Weissman, J. S., Biochemical basis of oxidative protein folding in the endoplasmic reticulum. Science 2000, 290 (5496), 1571-1574.

37. Weissman, J. S.; Kim, P. S., Reexamination of the folding of BPTI: predominance of native intermediates. Science 1991, 253 (5026), 1386-93.

38. Kibria, F. M.; Lees, W. J., Balancing conformational and oxidative kinetic traps during the folding of bovine pancreatic trypsin inhibitor (BPTI) with glutathione and glutathione disulfide. J. Am. Chem. Soc. 2008, 130 (3), 796-7.

39. Scheraga, H. A.; Wedemeyer, W. J.; Welker, E., Bovine pancreatic ribonuclease A: oxidative and conformational folding studies. Methods in Enzymology 2001, 341, 189-221. 
40. Hondal, R. J.; Marino, S. M.; Gladyshev, V. N., Selenocysteine in Thiol/DisulfideLike Exchange Reactions. Antioxidants \& Redox Signaling 2012, 18 (13), 16751689.

41. Arai, K.; Noguchi, M.; Singh, B. G.; Priyadarsini, K. I.; Fujio, K.; Kubo, Y.; Takayama, K.; Ando, S.; Iwaoka, M., A water-soluble selenoxide reagent as a useful probe for the reactivity and folding of polythiol peptides. FEBS Open Bio. 2013, 3, 55-64.

42. Beld, J.; Woycechowsky, K. J.; Hilvert, D., Selenoglutathione: efficient oxidative protein folding by a diselenide. Biochemistry 2007, 46 (18), 5382-90.

43. Huber, R. E.; Criddle, R. S., Comparison of the chemical properties of selenocysteine and selenocystine with their sulfur analogs. Archives of Biochemistry and Biophysics 1967, 122 (1), 164-173.

44. Metanis, N.; Hilvert, D., Harnessing selenocysteine reactivity for oxidative protein folding. Chem. Sci. 2015, 6 (1), 322-325.

45. Beld, J.; Woycechowsky, K. J.; Hilvert, D., Diselenides as universal oxidative folding catalysts of diverse proteins. Journal of Biotechnology 2010, 150 (4), 4819.

46. Gough, J. D.; Lees, W. J., Effects of redox buffer properties on the folding of a disulfide-containing protein: dependence upon $\mathrm{pH}$, thiol $\mathrm{pKa}$, and thiol concentration. J. Biotechnol. 2005, 115 (3), 279-90.

47. Gurbhele-Tupkar, M. C.; Perez, L. R.; Silva, Y.; Lees, W. J., Rate enhancement of the oxidative folding of lysozyme by the use of aromatic thiol containing redox buffers. Bioorg. Med. Chem. 2008, 16 (5), 2579-90.

48. Chang, J. Y., Diverse pathways of oxidative folding of disulfide proteins: underlying causes and folding models. Biochemistry 2011, 50 (17), 3414-31.

49. Rothwarf, D. M.; Li, Y. J.; Scheraga, H. A., Regeneration of bovine pancreatic ribonuclease A: identification of two nativelike three-disulfide intermediates involved in separate pathways. Biochemistry 1998, 37 (11), 3760-6.

50. Rothwarf, D. M.; Li, Y. J.; Scheraga, H. A., Regeneration of bovine pancreatic ribonuclease A: detailed kinetic analysis of two independent folding pathways. Biochemistry 1998, 37 (11), 3767-76.

51. $\mathrm{Xu}, \mathrm{X}$.; Scheraga, H. A., Kinetic folding pathway of a three-disulfide mutant of bovine pancreatic ribonuclease A missing the [40-95] disulfide bond. Biochemistry 1998, 37 (20), 7561-71. 
52. Gough, J. D.; Williams, R. H.; Donofrio, A. E.; Lees, W. J., Folding disulfidecontaining proteins faster with an aromatic thiol. Journal of the American Chemical Society 2002, 124 (15), 3885-3892.

53. Diamond, R., Real-space refinement of the structure of hen egg-white lysozyme. Journal of Molecular Biology 1974, 82 (3), 371-91.

54. Dong, X.-Y.; Huang, Y.; Sun, Y., Refolding kinetics of denatured-reduced lysozyme in the presence of folding aids. Journal of Biotechnology 2004, 114 (12), 135-142.

55. De Bernardez Clark, E.; Hevehan, D.; Szela, S.; Maachupalli-Reddy, J., Oxidative renaturation of hen egg-white lysozyme. Folding vs aggregation. Biotechnol Prog. 1998, $14(1), 47-54$.

56. van den Berg, B.; Chung, E. W.; Robinson, C. V.; Mateo, P. L.; Dobson, C. M., The oxidative refolding of hen lysozyme and its catalysis by protein disulfide isomerase. The EMBO Journal 1999, 18 (17), 4794-4803.

57. Ascenzi, P.; Bocedi, A.; Bolognesi, M.; Spallarossa, A.; Coletta, M.; Cristofaro, R.; Menegatti, E., The Bovine Basic Pancreatic Trypsin Inhibitor (Kunitz Inhibitor): A Milestone Protein. Current Protein and Peptide Science 2003, 4 (3), 231-251.

58. Creighton, T. E., Experimental studies of protein folding and unfolding. Progress in Biophysics and Molecular Biology 1979, 33, 231-297.

59. Creighton, T. E., The single-disulphide intermediates in the refolding of reduced pancreatic trypsin inhibitor. Journal of Molecular Biology 1974, 87 (3), 603-624.

60. Dadlez, M.; Kim, P. S., A third native one-disulphide intermediate in the folding of bovine pancreatic trypsin inhibitor. Nature Structural Biology 1995, 2 (8), 674-9.

61. Weissman, J. S.; Kim, P. S., A kinetic explanation for the rearrangement pathway of BPTI folding. Nat. Struct. Biol. 1995, 2 (12), 1123-30.

62. Creighton, T. E.; Weissman, J. S.; Kim, P. S., The disulfide folding pathway of BPTI. Science 1992, 256 (5053), 111-114.

63. Creighton, T. E.; Goldenberg, D. P., Kinetic role of a meta-stable native-like twodisulphide species in the folding transition of bovine pancreatic trypsin inhibitor. Journal of Molecular Biology 1984, 179 (3), 497-526.

64. Weissman, J. S.; Kim, P. S., Response. Science 1992, 256, 112-114.

65. Goldenberg, D. P., Kinetic analysis of the folding and unfolding of a mutant form of bovine pancreatic trypsin inhibitor lacking the cysteine-14 and -38 thiols. Biochemistry 1988, 27 (7), 2481-2489. 
66. Giorgio, R. P.; Barbara, V., Folding/unfolding/refolding of proteins: Present methodologies in comparison with capillary zone electrophoresis. Electrophoresis 2001, 22 (12), 2359-2374.

67. Bai, Y.; Sosnick, T. R.; Mayne, L.; Englander, S. W., Protein folding intermediates: native-state hydrogen exchange. Science 1995, 269 (5221), 192.

68. Englander, S. W.; Kallenbach, N. R., Hydrogen exchange and structural dynamics of proteins and nucleic acids. Quarterly Reviews of Biophysics 1983, 16 (4), 521655.

69. Krishna, M. M.; Hoang, L.; Lin, Y.; Englander, S. W., Hydrogen exchange methods to study protein folding. Methods (San Diego, Calif.) 2004, 34 (1), 51-64.

70. Wagner, G.; Wüthrich, K., Correlation between the amide proton exchange rates and the denaturation temperatures in globular proteins related to the basic pancreatic trypsin inhibitor. Journal of Molecular Biology 1979, 130 (1), 31-37.

71. Wüthrich, K.; Wagner, G., Nuclear magnetic resonance of labile protons in the basic pancreatic trypsin inhibitor. Journal of Molecular Biology 1979, 130 (1), 118.

72. Viswanatham, K.; T., C. B.; Steven, C., Conformational changes in proteins probed by hydrogen - exchange electrospray - ionization mass spectrometry. Rapid Communications in Mass Spectrometry 1991, 5 (4), 214-217.

73. Konermann, L.; Pan, J.; Liu, Y. H., Hydrogen exchange mass spectrometry for studying protein structure and dynamics. Chemical Society reviews 2011, 40 (3), 1224-34.

74. Werner, M. H.; Clore, G. M.; Gronenborn, A. M.; Kondoh, A.; Fisher, R. J., Refolding proteins by gel filtration chromatography. FEBS Letters 1994, 345 (2), 125-130.

75. Jungbauer, A.; Kaar, W.; Schlegl, R., Folding and refolding of proteins in chromatographic beds. Current Opinion in Biotechnology 2004, 15 (5), 487-494.

76. Gu, Z.; Weidenhaupt, M.; Ivanova, N.; Pavlov, M.; Xu, B.; Su, Z. G.; Janson, J. C., Chromatographic methods for the isolation of, and refolding of proteins from, Escherichia coli inclusion bodies. Protein Expression and Purification 2002, 25 (1), 174-9.

77. Creighton, T. E., Conformational restrictions on the pathway of folding and unfolding of the pancreatic trypsin inhibitor. Journal of Molecular Biology 1977, 113 (2), 275-93. 
78. Li, M.; Zhang, G.; Su, Z., Dual gradient ion-exchange chromatography improved refolding yield of lysozyme. Journal of Chromatography A 2002, 959 (1), 113-120.

79. Verzola, B.; Chiti, F.; Manao, G.; Righetti, P. G., Monitoring equilibria and kinetics of protein folding/unfolding reactions by capillary zone electrophoresis. Anal. Biochem. 2000, 282 (2), 239-244.

80. Hilser, V. J.; Worosila, G. D.; Freire, E., Analysis of Thermally Induced Protein Folding/Unfolding Transitions Using Free Solution Capillary Electrophoresis. Analytical Biochemistry 1993, 208 (1), 125-131.

81. Loo, J. A.; Edmonds, C. G.; Udseth, H. R.; Smith, R. D., Effect of reducing disulfide-containing proteins on electrospray ionization mass spectra. Anal. Chem. 1990, 62 (7), 693-698.

82. Jensen, P. K.; Harrata, A. K.; Lee, C. S., Monitoring protein refolding induced by disulfide formation using capillary isoelectric focusing-electrospray ionization mass spectrometry. Anal. Chem. 1998, 70 (10), 2044-2049.

83. DeCollo, T. V.; Lees, W. J., Effects of aromatic thiols on thiol-disulfide interchange reactions that occur during protein folding. J. Org. Chem. 2001, 66 (12), 4244-4249.

84. Maeda, Y.; Ueda, T.; Yamada, H.; Imoto, T., The role of net charge on the renaturation of reduced lysozyme by the sulfhydryl-disulfide interchange reaction. Protein Eng. 1994, 7 (10), 1249-54.

85. Doig, A. J.; Williams, D. H., Is the hydrophobic effect stabilizing or destabilizing in proteins?: The contribution of disulphide bonds to protein stability. Journal of Molecular Biology 1991, 217 (2), 389-398.

86. Houk, J.; Singh, R.; Whitesides, G. M., Measurement of thiol-disulfide interchange reactions and thiol pKa values. In Methods in enzymology, Academic Press: 1987; Vol. 143, pp 129-140.

87. Kemp, G., Capillary electrophoresis: a versatile family of analytical techniques. Biotechnology and Applied Biochemistry 1998, 27 ( Pt 1), 9-17.

88. Jorgenson, J. W.; Lukacs, K. D., Capillary zone electrophoresis. Science (New York, N.Y.) 1983, 222 (4621), 266.

89. Blanco, D.; Herrero, I.; Laviana, L.; Gutie`rrez, M. D., Capillary zone electrophoretic separation of proteins using coated capillaries. Journal of Liquid Chromatography \& Related Technologies 2002, 25 (8), 1171-1185.

90. Parks, G. A., The isoelectric points of solid oxides, solid hydroxides, and aqueous hydroxo complex systems. Chemical Reviews 1965, 65 (2), 177-198. 
91. Lucy, C. A.; MacDonald, A. M.; Gulcev, M. D., Non-covalent capillary coatings for protein separations in capillary electrophoresis. J. Chromatogr. A 2008, 1184 (1-2), 81-105.

92. Hajba, L.; Guttman, A., Recent advances in column coatings for capillary electrophoresis of proteins. TrAC Trends in Analytical Chemistry 2017, 90, 38-44.

93. McCormick, R. M., Capillary zone electrophoretic separation of peptides and proteins using low pH buffers in modified silica capillaries. Anal. Chem. 1988, 60 (21), 2322-2328.

94. Corradini, D.; Bevilacqua, L.; Nicoletti, I., Separation of basic proteins in bare fused-silica capillaries with diethylentriamine phosphate buffer as the background electrolyte solution. Chromatographia 2005, 62 (13), s43-s50.

95. Bushey, M. M.; Jorgenson, J. W., Capillary electrophoresis of proteins in buffers containing high concentrations of zwitterionic salts. Journal of Chromatography 1989, 480, 301-10.

96. Stellwagen, E.; Prantner, J. D.; Stellwagen, N. C., Do zwitterions contribute to the ionic strength of a solution? Analytical Biochemistry 2008, 373 (2), 407-409.

97. Horvath, J.; Dolník, V., Polymer wall coatings for capillary electrophoresis. Electrophoresis 2001, 22 (4), 644-655.

98. Verzola, B.; Gelfi, C.; Righetti, P. G., Quantitative studies on the adsorption of proteins to the bare silica wall in capillary electrophoresis. II. Effects of adsorbed, neutral polymers on quenching the interaction. Journal of Chromatography. A $\mathbf{2 0 0 0}$, 874 (2), 293-303.

99. He, Y.; Lacher, N. A.; Hou, W.; Wang, Q.; Isele, C.; Starkey, J.; Ruesch, M., Analysis of identity, charge variants, and disulfide isomers of monoclonal antibodies with capillary zone electrophoresis in an uncoated capillary column. Anal. Chem. 2010, 82 (8), 3222-30.

100. He, Y.; Isele, C.; Hou, W.; Ruesch, M., Rapid analysis of charge variants of monoclonal antibodies with capillary zone electrophoresis in dynamically coated fused-silica capillary. Journal of Separation Science 2011, 34 (5), 548-555. 


\section{NA ZHANG}

\section{Born, Xi'an, China}

2006-2010 B.E., The National Base of Life Science and Biotechnology Education China Pharmaceutical University, Nanjing, China

2010-2012 M.S., Microbial and Biochemical Pharmacy China Pharmaceutical University, Nanjing, China

2013-2018 Doctoral Candidate

Florida International University, Miami, Florida

2013-2018 Teaching Assistant

Florida International University, Miami, Florida

\section{PUBLICATIONS AND PRESENTATIONS}

Zhang N.; Marahatta R.P.; and Lees W.J., (2018) Folding analysis of bovine pancreatic trypsin inhibitor (BPTI) with aromatic thiols and disulfides in vitro. 255 ${ }^{\text {th }}$ American Chemical Society National Meeting \& Exposition. New Orleans, LA., March 18-22, 2018.

Zhang N.; Marahatta R.P.; Moats M.A.; and Lees W.J., (2017) In vitro oxidative folding of disulfide-containing protein using aromatic thiols and disulfides. 253 $3^{\text {rd }}$ American Chemical Society National Meeting \& Exposition. San Francisco, CA., April 2-6, 2017.

Zhang N.; Marahatta R.P.; and Lees W.J., (2016) Oxidative folding of reduced bovine pancreatic trypsin inhibitor with aromatic thiols and the corresponding disulfides. $92^{\text {nd }}$ Florida Annual Meeting and Exhibition. Tampa, FL., May 5-7, 2016.

Zhang N.; Yao W, and Xu C., (2012) Journal of International Pharmaceutical Research. 39(2): 137-142. 